\title{
Impact of Chronic Conditions on Treatment, Cancer-and Non- Cancer Outcomes among Elderly Men with Incident Prostate Cancer
}

Amitkumar Dineshchandra Raval

Follow this and additional works at: https://researchrepository.wvu.edu/etd

\section{Recommended Citation}

Raval, Amitkumar Dineshchandra, "Impact of Chronic Conditions on Treatment, Cancer-and Non-Cancer Outcomes among Elderly Men with Incident Prostate Cancer" (2015). Graduate Theses, Dissertations, and Problem Reports. 6483.

https://researchrepository.wvu.edu/etd/6483

This Dissertation is protected by copyright and/or related rights. It has been brought to you by the The Research Repository @ WVU with permission from the rights-holder(s). You are free to use this Dissertation in any way that is permitted by the copyright and related rights legislation that applies to your use. For other uses you must obtain permission from the rights-holder(s) directly, unless additional rights are indicated by a Creative Commons license in the record and/ or on the work itself. This Dissertation has been accepted for inclusion in WVU Graduate Theses, Dissertations, and Problem Reports collection by an authorized administrator of The Research Repository @ WVU.

For more information, please contact researchrepository@mail.wvu.edu. 


\title{
Impact of Chronic Conditions on Treatment, Cancer-and Non-Cancer Outcomes among Elderly
} Men with Incident Prostate Cancer

\author{
Amitkumar Dineshchandra Raval \\ Dissertation submitted \\ to the School of Pharmacy \\ at West Virginia University \\ in partial fulfillment of the requirements for the degree of \\ Doctor of Philosophy in \\ Health Outcomes Research \\ Usha Sambamoorthi, Ph.D., Chair \\ S.Suresh Madhavan, M.B.A, Ph.D. \\ Malcolm Mattes, M.D. \\ Wenhui Wei, MBA, Ph.D. \\ Xiaoyun Pan, Ph.D. \\ Department of Pharmaceutical Systems and Policy \\ Morgantown, West Virginia \\ 2015
}

Keywords: Prostate Cancer, Cardio-metabolic conditions, Metformin, Hospitalizations

Copyright 2015 Amitkumar Dineshchandra Raval 


\title{
ABSTRACT \\ Impact of Chronic Conditions on Treatment, Cancer-and Non-Cancer Outcomes among Elderly Men with Incident Prostate Cancer
}

\author{
Amitkumar Dineshchandra Raval
}

Prostate Cancer is the most commonly observed non-skin cancer among the elderly men aged 65 years and older in the United States. Nearly one third of elderly men diagnosed with incident prostate cancer have pre-existing chronic conditions. Therefore, among elderly men with prostate cancer, management for cancer and chronic conditions should be optimized to improve healthcare outcomes. Previous literature majorly focused on the risk and management of cancer in the presence of number of conditions, although, it is known that more than $70 \%$ of chronic conditions among men diagnosed with prostate cancer were either cardio-metabolic, respiratory or mental health conditions. Lack of evidence persists regarding the impact of common types of chronic conditions and their conditions among elderly men with prostate cancer and vice-versa. The current study is an attempt to shrink the knowledge gap to provide actionable strategies to better management of chronic conditions and prostate cancer among elderly men. The three specific aims of the study were to: (1) examine the associations between the types of pre-existing chronic conditions and cancer stage at diagnosis, initial cancer treatment and clinical outcomes after initial cancer treatment; 2) examine the relationship between metformin use and cancer stage at diagnosis, and the initial cancer-treatment; 3) analyze the impact of cancer diagnosis on the risk of non-cancer hospitalizations and evaluate whether the impact of cancer diagnosis on the risk of non-cancer hospitalizations vary by the types of pre-existing chronic conditions among fee-for-service elderly Medicare beneficiaries with incident prostate cancer. The study used a retrospective cohort design, using multiple years (2002-2010) of the cancer registry data from the Surveillance, Epidemiology and End Results (SEER) program linked with the Medicare administrative claims data and the Area Health Resource Files (AHRF). In the first aim, among elderly men with incident prostate cancer $(\mathrm{N}=103,820)$, the cardio-vascular conditions were the most common chronic condition. 1 in 10 elderly men had advanced prostate cancer at diagnosis. Elderly men without cardio-metabolic, respiratory or mental health conditions were more likely to be diagnosed with advanced prostate cancer as compared to those with all the three types of chronic conditions. 3 in 4 elderly men with localized prostate cancer received either radical prostatectomy (RP), radiation therapy (RT) or hormone therapy during the first six-month after cancer diagnosis. As compared to all three types of chronic conditions, those with single types of chronic conditions were less likely to develop bowel, and urinary dysfunctions. In the second aim, the use of metformin was associated with a reduction in the risk of advanced prostate cancer among elderly men diagnosed with prostate cancer and pre-existing diabetes $(\mathrm{N}=2,652)$. In the third study, elderly men diagnosed with prostate cancer had an increase in the risk of non-cancer hospitalizations during the post-cancer period as compared to the pre-cancer period in both unadjusted and adjusted analyses. The highest rates of non-cancer hospitalizations were observed during first four months after the diagnosis of prostate cancer. To summarize, our study confirms that elderly men with incident prostate cancer and multiple types of pre-existing chronic conditions would pose a different degree of risk for the development of advanced prostate cancer. Although the management of chronic conditions such diabetes with metformin may reduce the risk of advanced prostate cancer among elderly men. An overuse of RT/RP in men with different types of chronic conditions and an increase in the non-cancer hospitalizations in the initial period after diagnosis of prostate cancer suggest the scope of optimum use of RT and RP and improvement in the care of chronic conditions. 
DEDICATION

This dissertation is dedicated to my late father Dineshchandra Ratilal Raval (20/5/1952-4/12/1989) who was a victim of cancer. 
I thank Dr. Usha Sambamoorthi for not only serving as dissertation committee chair, but also being an excellent mentor. I was really blessed to have Dr. Sambamoorthi as my new committee chair after my previous advisor left WVU. Dr. Sambamoorthi's style of in-person couching on the analytic and reasoning skills as well as detailed oriented work helped me to solve complex analytic problems and execute difficult tasks with ease. Without her support, I would not have completed this dissertation.

I want to thank my other committee members for their kind support and wise advice. Dr. Suresh Madhavan introduced me to cancer survivorship research and provided many helpful suggestions as this study was supported by his 24HS018622-03 grant from the Agency for Healthcare Research and Quality. I am also thankful to him for providing data access for this project. Dr. Malcolm Mattes's insightful comments and clinical expertise helped me to put this study into perspective. I am grateful to Dr. Xiaoyun Pan and Dr. Wenhui Wei for the guidance in advanced statistical analysis techniques.

I also want to acknowledge the support of the West Virginia (WV) Cohort Center data analyst, Sita Kalidindi and senior students (Pallavi Rane, Ami Vyas, Pramit Nadapara and Neel Shah) who worked on the similar areas under Agency for Healthcare Research \& Quality (AHRQ). Their research work not only sensitized ideas for my dissertation, but initial work of them helped me to better understand the cancer registry data and execution of various programs to create my analytic files for my dissertation. I would also like to thank the WVU family: Dr. Kimberly Kelly, Dr. Ginger Scott, Dr. Gerald Higa, Angie Frame, and all the graduate students for their support and making my time spent at WVY very memorable.

Finally, although words are not enough to thank one's family and friends, I want to thank my mom for her sacrifices she made for me to make me what I am today. I also want to acknowledge love, and support of my sister, brother-in-law and my fiancé during high and low during my graduate studies. 
Table of Contents

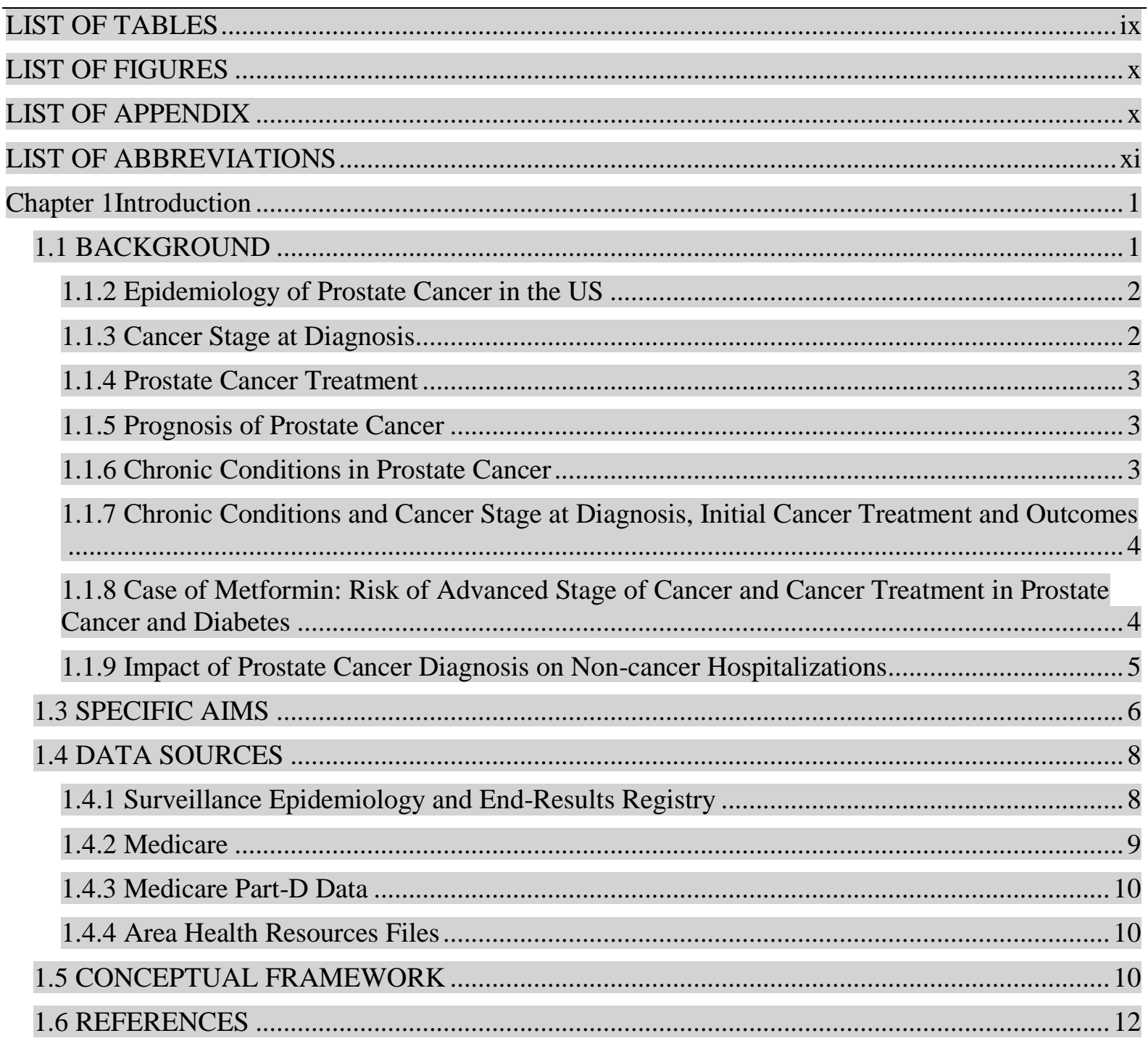

Chapter 2 Types of Chronic Conditions and Cancer Stage at Diagnosis, Initial Cancer Treatment and Clinical Outcomes among Elderly Medicare Beneficiaries with Prostate Cancer ............................... 16

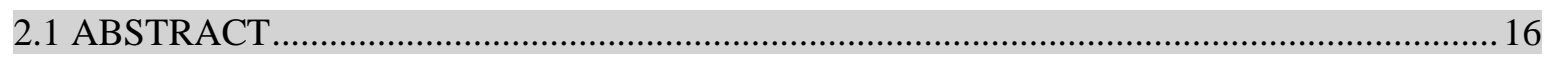

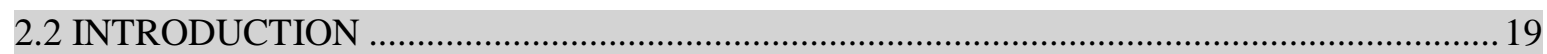

2.2.1 Pre-existing Chronic Conditions and Cancer Stage at Diagnosis ...................................... 19

2.2.2 Pre-existing Chronic Conditions and Prostate Cancer Treatment.......................................... 20

2.2.3 Number or Weighted Score of Chronic Conditions ...................................................... 21

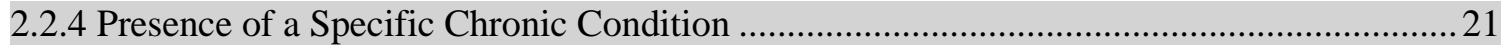

2.2.5 Preexisting Chronic Conditions and Clinical outcomes....................................................... 22

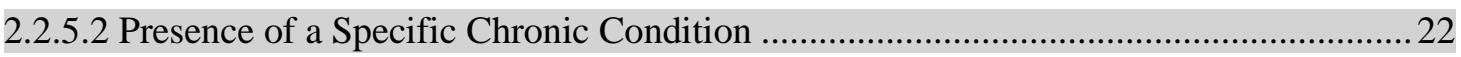

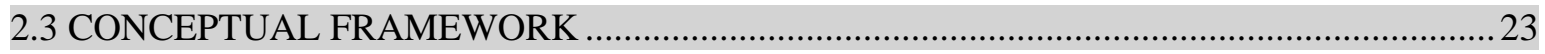

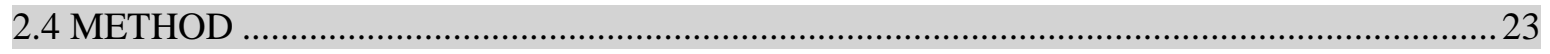

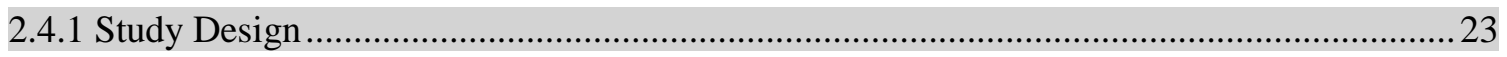

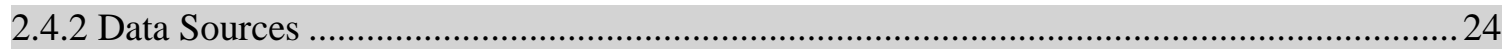

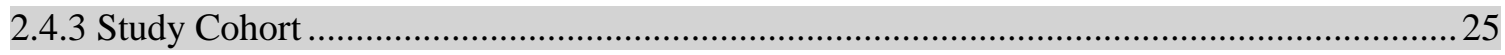


2.4.4 Key Dependent Variables 2.4.4.1 Prostate Cancer Stage at Diagnosis (Localized and Advanced Stage)

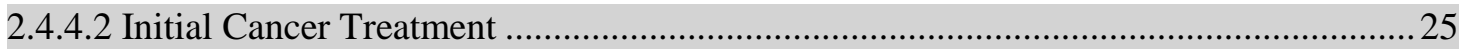

2.4.4.3 Clinical Outcomes-Urinary, bowel, and sexual dysfunction..........................................26

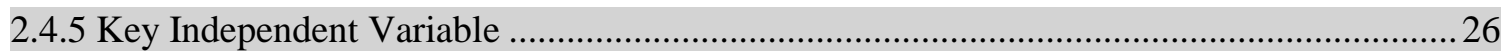

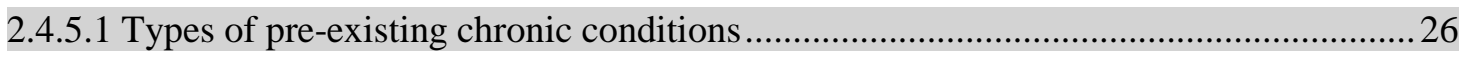

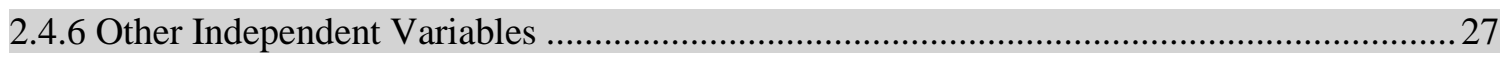

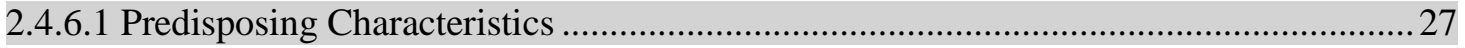

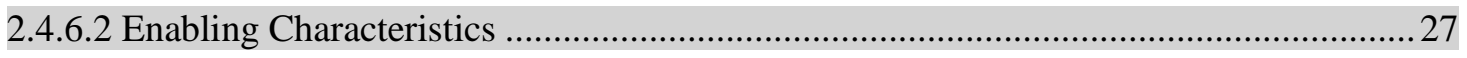

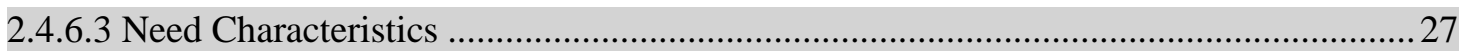

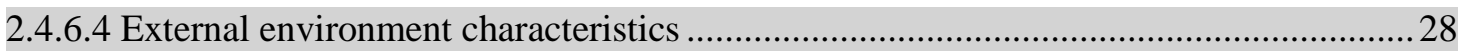

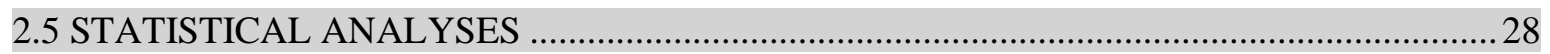

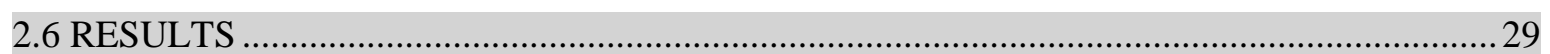

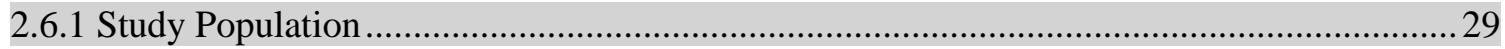

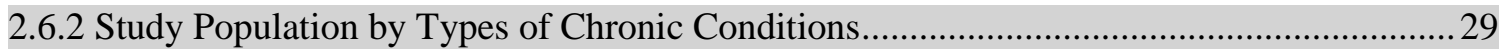

2.6.3 Types of Chronic Conditions and Cancer Stage at Diagnosis ............................................ 30

2.6.4 Types of Chronic Conditions and Initial Cancer Treatment Type ….................................... 30

2.6.5 Types of Chronic Conditions and Clinical Outcomes ...................................................... 31

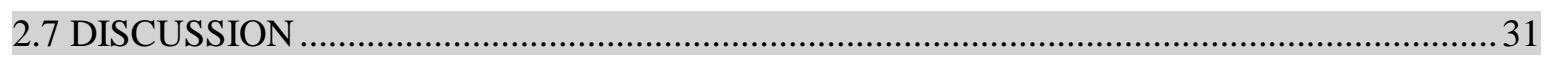

2.7.1 Types of Chronic Conditions and Cancer Stage at Diagnosis ............................................ 32

2.7.2 Types of Chronic Conditions and Initial Cancer Treatment .............................................. 32

2.7.3 Types of Chronic Conditions and Clinical Outcomes....................................................... 34

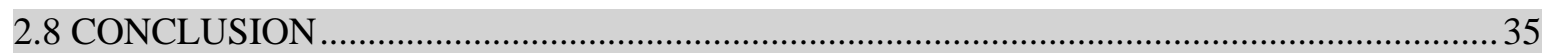

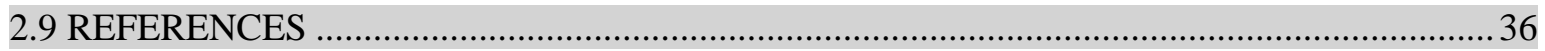

Chapter 3 Association between Metformin Use and Cancer Stage at Diagnosis and Initial Cancer Treatment among Elderly Medicare Beneficiaries with Diabetes and Prostate Cancer .......................51

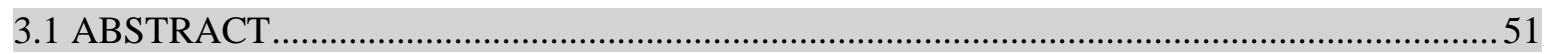

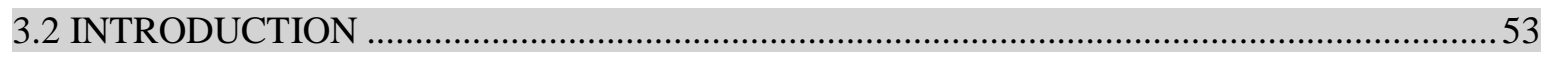

3.2.1 Diabetes and Prostate Cancer Risk and Stage at Diagnosis .............................................53

3.2.2 Anti-diabetes Drugs (Metformin) and Cancer Stage at Diagnosis ....................................... 53

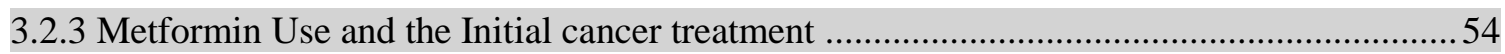

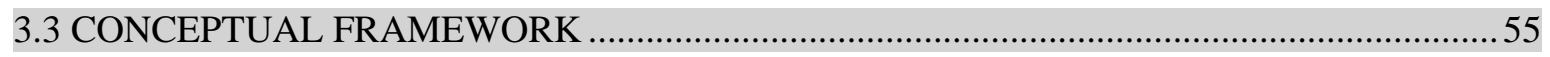

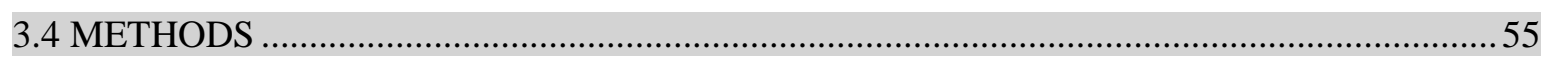

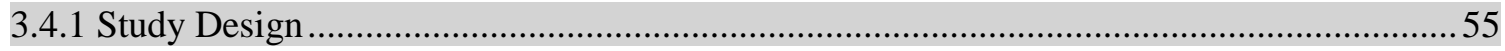

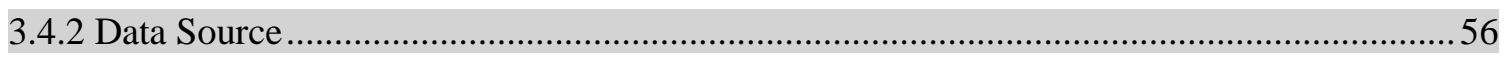

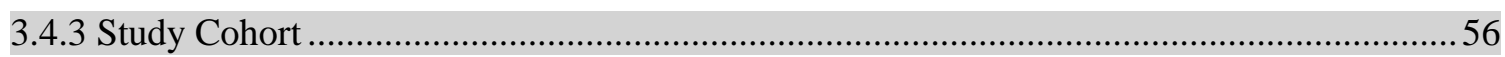

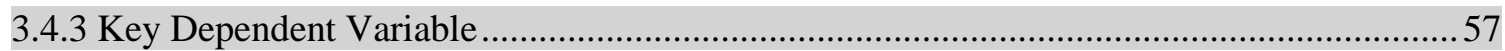

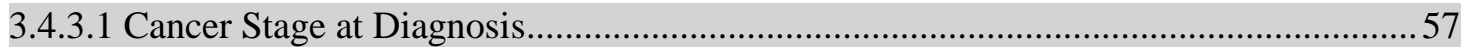

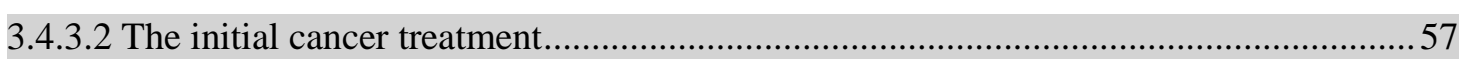

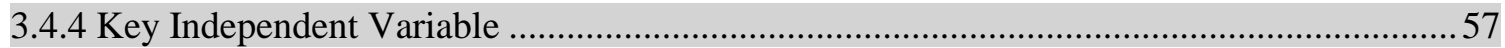


3.4.4.1 Metformin Use.

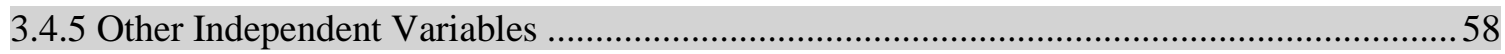

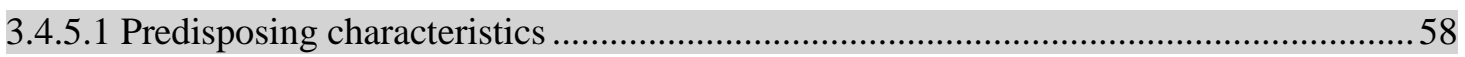

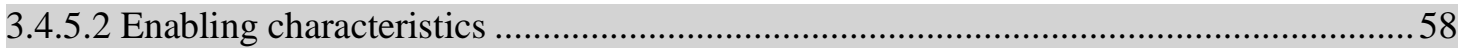

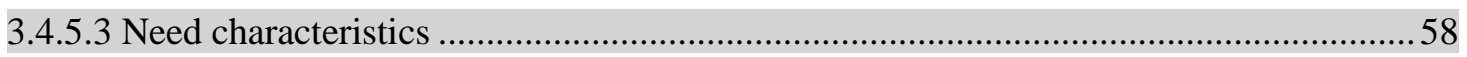

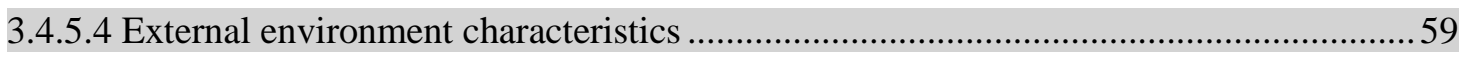

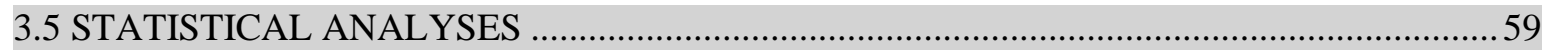

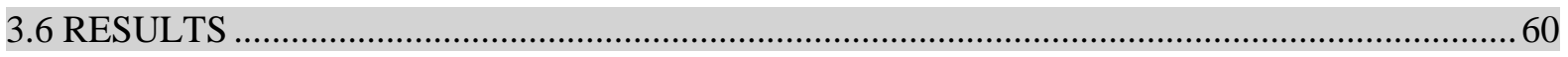

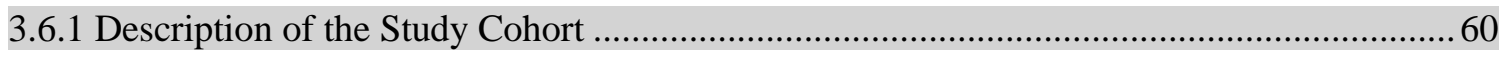

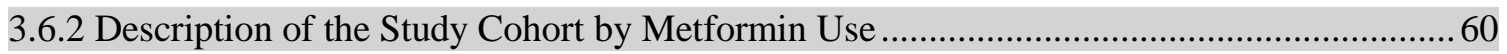

3.6.3 Metformin and Cancer Stage at Diagnosis..................................................................... 60

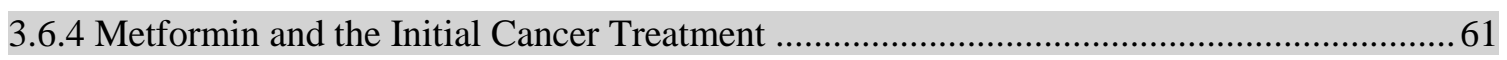

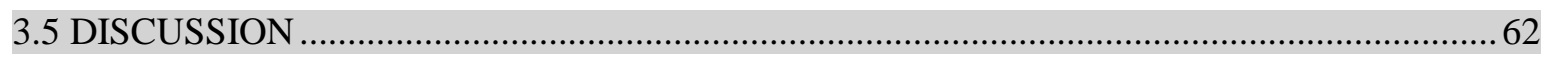

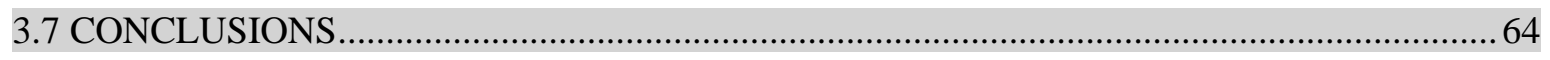

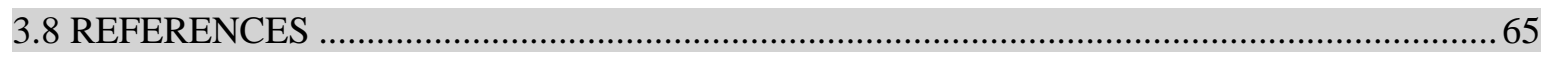

Chapter 4 Impact of Prostate Cancer Diagnosis on Non-Cancer Hospitalizations by Types of Chronic Conditions among Elderly Medicare Beneficiaries with Incident Prostate Cancer................................ 75

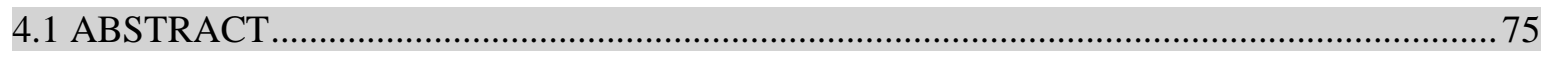

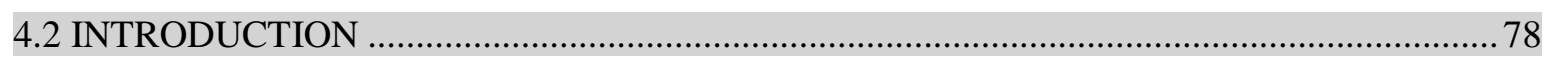

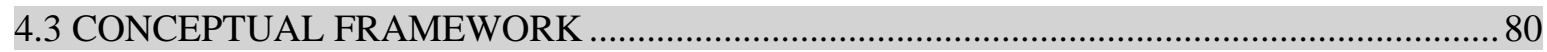

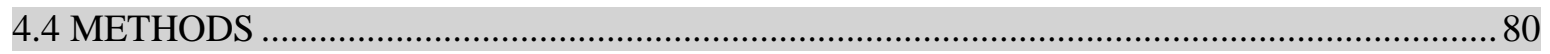

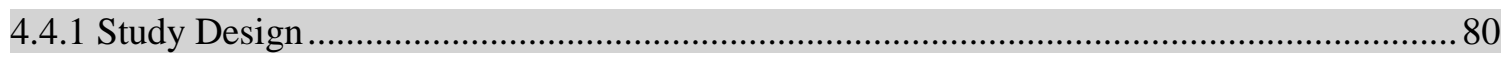

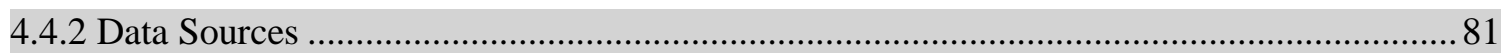

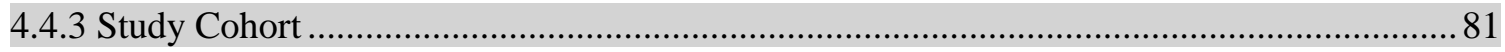

4.4.4 Key Dependent Variable: Non-cancer hospitalizations ..................................................... 81

4.4.5 Key Independent Variable: Types of co-occurring chronic condition (One of the need

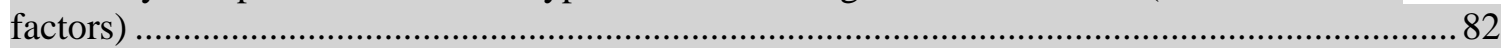

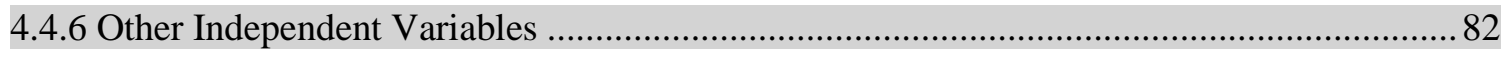

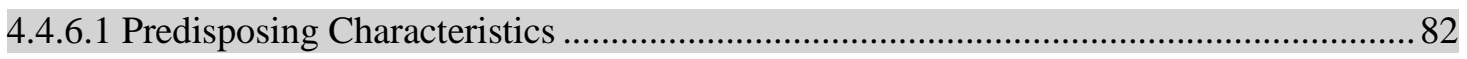

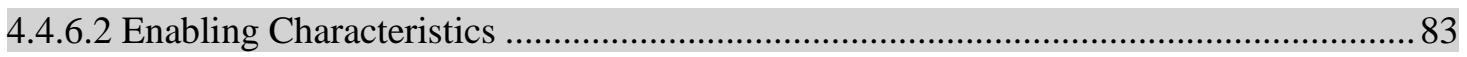

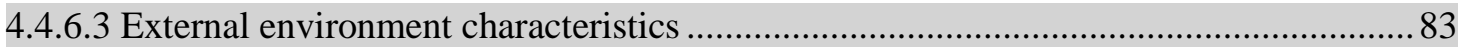

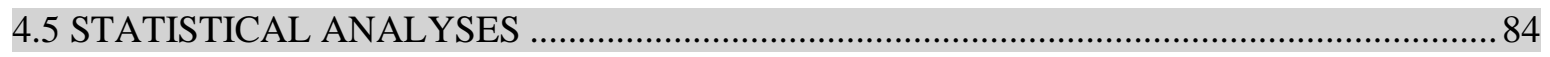

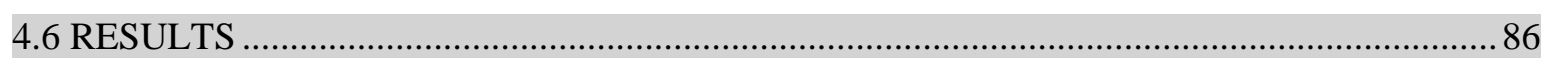

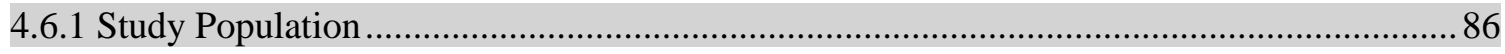

4.6.2 Non-cancer-Hospitalizations during the pre- and post-cancer period................................... 86

4.6.3 Probabilities of Non-cancer hospitalizations by Pre and Post-Cancer Period........................ 87

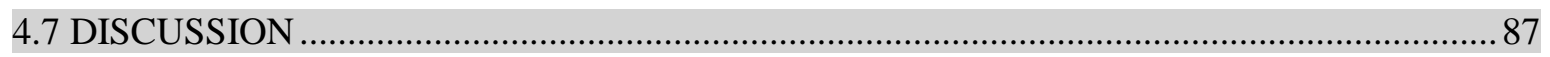

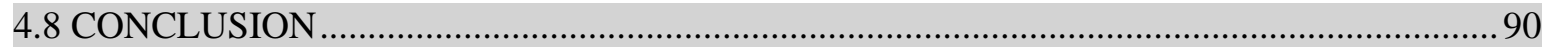

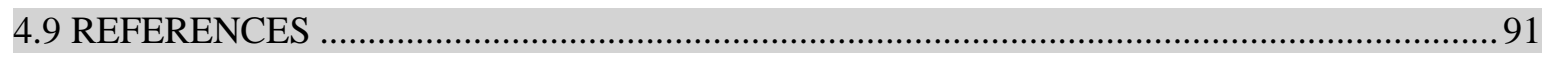

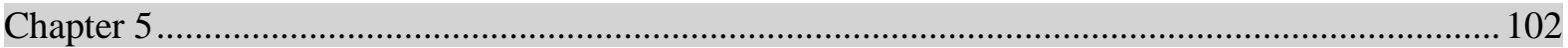




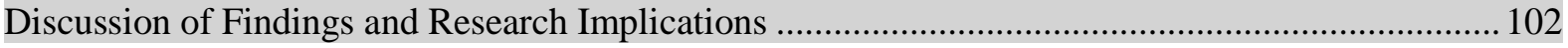

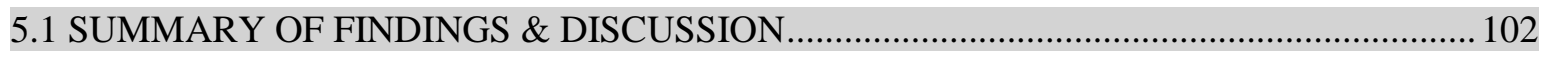

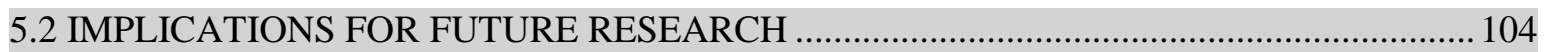

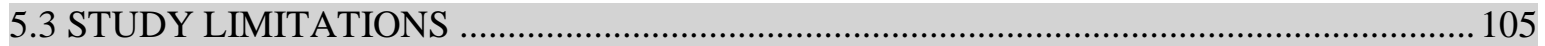

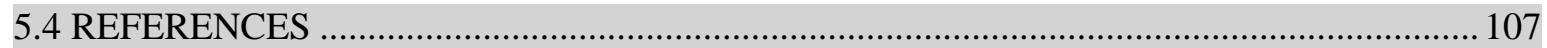


LIST OF TABLES

Table 1.1 American Joint Committee on Cancer (AJCC) Clinical TNM Classification of Prostatic Tumors

Table 1.2 Details of Variables for Each Study Objectives-classified based on ABM

Table 2.1 Characteristics of Elderly Medicare Beneficiaries with Prostate Cancer Surveillance, Epidemiology and End Results (SEER)-Medicare Linked Database-2002-2010.

Table 2.2 Characteristics of Elderly Medicare Beneficiaries with Prostate Cancer by Types of Chronic Conditions

Table 2.3 Number and Percent with Advanced Prostate Cancer and Adjusted Odds Ratios (AOR) and 95\% Confidence Intervals from Logistic Regressions for Advanced Stage Diagnosis among Elderly Medicare Beneficiaries with Prostate Cancer Surveillance, Epidemiology and End Results (SEER)Medicare Linked Database 2002-2010 .

Table 2.4 Number and Percentage with Initial Cancer Treatment by Types of Chronic Conditions Unadjusted and Adjusted Odds Ratios and 95\% Confidence Intervals from Multinomial Logistic Regressions among Elderly Medicare Beneficiaries with Prostate Cancer Surveillance, Epidemiology and End Results (SEER)-Medicare Linked Database 2002-2010.

Table 2.5 Number and Percentage with Poor Clinical Outcomes Unadjusted and Adjusted Odds Ratios and 95\% Confidence Intervals from Logistic Regressions of Clinical Outcomes among Elderly Men with Prostate Cancer by Types of Chronic Conditions Surveillance, Epidemiology and End Results-Medicare Linked Database 2002-2010

Table 3.1 Characteristics of the Study Cohort Elderly Medicare Beneficiaries with Diabetes and Incident Prostate Cancer by Metformin Use SEER-Medicare-linked Database 2007- 2010.

Table 3.2 Adjusted Odds Ratios and 95\% Confidence Intervals from Logistic Regressions On Metformin Use among Elderly Medicare Beneficiaries with diabetes Incident Prostate Cancer Surveillance, Epidemiology and End Results (SEER)-Medicare Linked Data-2007-2010

Table 3.3 Number and IPTW-Adjusted Percentage of Men with Localized Versus and Advanced Stage of Prostate Cancer by Metformin Use Elderly Medicare Beneficiaries with Diabetes and Incident Prostate Cancer Surveillance, Epidemiology and End Results (SEER)-Medicare Linked Database-2007-2010

Table 3.4 Numbers and IPTW-Adjusted Percentages of the Initial Cancer Treatment Categories ..... 71

Table 4.1 Characteristics of Elderly Medicare Beneficiaries with Incident Prostate Cancer Surveillance, Epidemiology and End Results (SEER)- Medicare Linked Database 2000 - 2010 .93

Table 4.2 Number and Percentage with Non-Cancer Related Hospitalizations during the Pre- and Post-Cancer Period.

Table 4.3 Adjusted Odds Ratios and 95\% Confidence Intervals (CI) of Cancer Diagnosis Period From GEE models on Non-Cancer Related Hospitalizations Elderly Men with Incident Prostate Cancer Surveillance, Epidemiology and End Results (SEER)-Medicare Linked Database-2000-2010

Table 4.4 Predicted Probabilities with 95\% Confidence Intervals for Types of Chronic Conditions From GEE analysis on Non-Cancer Related Hospitalizations Elderly Medicare Beneficiaries with Incident Prostate Cancer Surveillance, Epidemiology and End Results (SEER)-Medicare Linked Database-2000-2010 


\section{LIST OF FIGURES}

Figure 2.1 Study Theoretical Framework: Adaptation of Andersen Behavioral Model for Aim 1 ......23

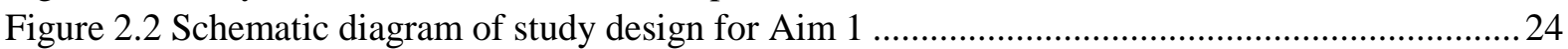
Figure 3.1: Study Theoretical Framework: Adaptation of Andersen Behavioral Model for Aim 2 .....55 Figure 3. 2 Schematic Presentation of Study Design for Aim 2 …................................................56 Figure 4. 1 Study Theoretical Framework: Adaptation of Andersen Behavioral Model for Aim 3 ..... 80 Figure 4. 2 Schematic Presentation of Study Design for Aim 3 ........................................................ 81

\section{LIST OF APPENDIX}

Appendix 2.1 Study Cohort Development Flow diagram for Study Population of Elderly Medicare Beneficiaries diagnosed with Prostate Cancer.

Appendix 2.2 Codes to Identify Radiation Therapy, Surgery, Hormonal and Chemotherapy

Utilization Among Elderly Medicare Beneficiaries with Prostate Cancer Using Surveillance, Epidemiology and End Results-Medicare Linked Data 2002-2010

Appendix 2.3 Diagnostic and Procedure codes used to Identify Clinical Outcomes-Bowel, Sexual and Urinary Dysfunctions Among Elderly Men with Prostate cancer using SEER-Medicare data from the year 2002 to 2010

Appendix 2.4 Codes Used to Identify the Types of Chronic Conditions and List of Other Chronic Conditions Among Elderly Medicare Beneficiaries with Incident Prostate Cancer Surveillance, Epidemiology, End-Results-Medicare Linked Data of 2002 to 2009.

Appendix 3.1 Study Cohort Development Flow diagram for Study Population of Elderly Medicare Beneficiaries diagnosed with Prostate Cancer and Diabetes

Appendix 3.2 Codes and Algorithms to Identify Diabetes Severity Diabetes Complication Severity Index (DCSI) developed by Young et al. and modified by Chang et al.

Appendix 4. 1 Study Cohort Development Chart for Study Population of Elderly Medicare Beneficiaries diagnosed with Prostate Cancer. . .98

Appendix 4. 2 Sensitivity Analysis: Non-cancer hospitalization definition 2-Number and Percentage with Non-Cancer Related Hospitalizations during the Pre- and Post-Cancer Period Elderly Medicare beneficiaries with Incident Prostate Cancer Surveillance, Epidemiology and End Results (SEER)Medicare Linked Database-2002-2010

Appendix 4. 3 Sensitivity Analysis: Non-cancer hospitalization definition 2-Adjusted Odds Ratios and 95\% Confidence Intervals (CI) of Cancer Diagnosis Period From GEE models on Non-Cancer Related Hospitalizations Elderly Men with Incident Prostate Cancer Surveillance, Epidemiology and End Results (SEER)-Medicare Linked Database-2000-2010

Appendix 4. 4 Sensitivity Analysis: Non-cancer hospitalization definition 2-Predicted Probabilities with 95\% Confidence Intervals for Types of Chronic Conditions From GEE analysis on Non-Cancer Related Hospitalizations Elderly Medicare Beneficiaries with Incident Prostate Cancer Surveillance, Epidemiology and End Results (SEER)-Medicare Linked Database-2000-2010 


\section{LIST OF ABBREVIATIONS}

\begin{tabular}{|c|c|}
\hline ADT & Androgen Deprivation Therapy \\
\hline AHRF & Area Healthcare Resource File \\
\hline AJCC & American Joint Committee on Cancer \\
\hline AMPK & Adenosine Monophosphate-activated Protein Kinase \\
\hline ASCVD & Atherosclerotic Cardiovascular Disease \\
\hline AUA & American Urology Association \\
\hline CCI & Charlson Comorbidity Index \\
\hline CI & Confidence Interval \\
\hline $\mathrm{CM}$ & Cardii-metabolic (conditions) \\
\hline CMS & Center for Medicare and Medicaid \\
\hline COPD & Chronic Obstructive Pulmonary Disease \\
\hline CSME & Cystoid Macular Edema/Degeneration \\
\hline DCSI & Diabetes Complications Severity Index \\
\hline DHHS & Department of Health and Human Services \\
\hline DME & Durable Medical Equipment \\
\hline FIPS & Federal Information Processing Standard \\
\hline GEE & Generalized Estimating Equations \\
\hline HHA & Home Health Agencies \\
\hline HMO & Health Maintenance Organizations \\
\hline ICD-9 CM & International Classification of Diseases, Ninth Revision, Clinical Modification \\
\hline IHD & Ischemic Heart Disease \\
\hline IOM & Institute of Medicine \\
\hline IPTW & Inverse Probability Treatment Weights \\
\hline LE & Lower Extremity \\
\hline LHRH & Luteinizing Hormone Releasing hormone \\
\hline MEDPAR & Medicare Provider Analysis and Review \\
\hline MH & Mental Health (conditions) \\
\hline mTOR & Mammalian Target of Rapamycin \\
\hline $\mathrm{NCCN}$ & National Cancer Comprehensive Network \\
\hline NDC & National Drug Codes \\
\hline NOS & Not Otherwise Specified \\
\hline OCS & Office of Cancer Survivorship \\
\hline OR & Odds Ratio \\
\hline OUTPT & Outpatient \\
\hline Pca & Prostate Cancer \\
\hline PCP & Primary Care Physician \\
\hline PDE & Part D Event \\
\hline PEDSF & Patient Entitlement and Diagnosis Summary File \\
\hline PSA & Prostate Specific Antigen \\
\hline PVD & Peripheral Vascular Disease \\
\hline QIC & Quasi-likelihood Information Criterion \\
\hline RESP & Respiratory (conditions) \\
\hline RP & Radical Prostatectomy \\
\hline RT & Radiation Therapy \\
\hline SAS & Statistical Analysis System \\
\hline SEER & Surviellance Epidemiology End-Results \\
\hline Sig. & Level of Significance \\
\hline T2DM & Type 2 Diabetes Mellitus \\
\hline TIA & Transient Ischemic Attack \\
\hline TNM & Tumor, Nodes, and Metastasis Staging System \\
\hline US & United States \\
\hline
\end{tabular}




\section{Chapter 1}

\subsection{BACKGROUND}

Many elderly men (age $\geq 65$ years) in the United States (US) have pre-existing chronic conditions when diagnosed with cancer. The presence of pre-existing chronic conditions is associated with poor quality of life and cancer-related outcomes. But limited evidence exists on how particular types of chronic conditions affect the risk of aggressive cancer, cancer treatment and clinical outcomes among elderly men with cancer. The management of some of the pre-existing chronic conditions may have beneficial effects on cancer care. For example, the management of pre-existing diabetes with metformin may not only improve the outcomes of diabetes, but also reduce the risk of cancer incidence or prevent aggressive cancers. Conversely, the diagnosis of cancer can be eclipse the management of pre-existing chronic conditions and affect non-cancer outcomes such as hospitalizations not related to the cancer. To date, no population-based study has addressed this issue among elderly men with cancer.

Therefore, the overall goal of the dissertation is to analyze the influence of pre-existing chronic conditions on cancer stage, cancer treatment, cancer-related clinical outcomes, and non-cancer outcomes (example: hospitalizations), and analyze the impact of pharmacological agents (example: metformin) used to manage pre-existing conditions (example: diabetes) on the cancer stage and the treatment for cancer. For the purposes of this dissertation, elderly men with prostate cancer was selected because of it is the leading cancer in men accounting for one fourth of all cancer cases diagnosed in the US.

The dissertation is organized as follows: Chapter 1 briefly describes the epidemiology of prostate cancer in the US, the prevalence of pre-existing chronic conditions among men with prostate cancer, the need for the study, conceptual framework for the study, and data sources for the study. Chapter 2 focuses on the association between the types of chronic conditions and stage of diagnosis, the treatment for prostate cancer and clinical outcomes. Chapter 3 emphases on the relationship management of pre-existing condition, specifically type 2 diabetes mellitus (T2DM) on the stage of cancer diagnosis and the treatment for cancer. In this chapter, the management of type 2 diabetes 
mellitus (T2DM) with metformin, as the first-line treatment for diabetes on the stage of cancer and the cancer treatment is analyzed. The chapter 4 highlights the relationship between pre-existing chronic conditions and non-cancer hospitalizations. Chapter 5 summarizes the study findings from the chapters 2,3 , and 4, and includes a discussion of the findings, implications and recommendations for future research. The advantages and limitations of the study findings are also included.

\subsubsection{Epidemiology of Prostate Cancer in the US}

Prostate cancer is a cancer of a gland surrounding the neck of the urinary bladder in men. It is the most common non-cutaneous (non-skin) cancer among men in the US. As of 2015, nearly 2.9 million prostate cancer survivors are alive, and about 222,800 new cases of prostate cancer are expected in 2015. Prostate cancer accounts for $24 \%$ of all cancer cases (1). Nearly two thirds of prostate cancer cases are diagnosed among the elderly men aged 65 years and older (2).

\subsubsection{Cancer Stage at Diagnosis}

Prostate cancer is often diagnosed at an earlier stage. The American Joint Committee on Cancer (AJCC) defines the stage of Prostate cancer by the Tumor, Nodes, and Metastasis (TNM) Staging System. The TNM system classifies a stage based on the spread of tumors to the local area, node, or metastases to other organs. "T" denotes for the extent of tumors in the prostate gland, "N" denotes spread of cancer to lymph nodes and " $\mathrm{M}$ " indicates the absence or presence of distant metastasis to bone, lung, liver or brain. Early stage tumors are confined to the prostate gland with no spread of cancer to the seminal vesicles or the lymph nodes or metastases (T1-T2, N0, M0). These types of cancers are also termed as localized or organ-confined cancer. Four in every five incident prostate cases $(80 \%)$ are diagnosed at the localized stage of prostate cancer.

Locally advanced prostate cancer, i.e. aggressive types of prostate cancer usually spreads to nearby regions of the prostate, including seminal vesicles, pelvic nodes, neck of the bladder or back passage or metastasis in bone or other organs (T3-T4, N1, M1). Less than one in every five incident prostate cancer cases are diagnosed as advanced prostate cancer. Details of the TNM staging for the prostate cancer are provided in Table $1.1(3)$. 


\subsubsection{Prostate Cancer Treatment}

Treatment options for prostate cancer vary depending on age, cancer stage, and the presence of serious chronic conditions. Traditional treatment modalities for prostate cancer include surgery to remove the tumors (radical prostatectomy - RP), radiation therapy (RT), and hormone therapy. The use of aggressive therapies such as RP or RT can lead to adverse clinical outcomes such as the bowel, sexual, urinary dysfunction. Further, due to a prolonged survival and early stage diagnosis of prostate cancer, men with prostate cancer can survive up to 10 years without even any aggressive treatment. Therefore, the treatment guideline recommends no use of aggressive treatment among those with limited survival benefits to avoid risk of adverse effects of treatment (4).

\subsubsection{Prognosis of Prostate Cancer}

With an estimated 27,540 deaths in 2015, prostate cancer is the second-leading cause of cancer death in men. However, in the last decade, the mortality rates among men with prostate cancer have declined. (1). In fact, men diagnosed with prostate cancer have a good survival profile with10year relative survival rates (compared to non-cancer cases) range: 90-100\%, even without aggressive treatments $(\mathrm{RP} / \mathrm{RT})$ if diagnosed as localized prostate cancer. However, men with advanced prostate cancer have a very poor survival profile with 5-year survival rate of only $23 \%$ (1). Therefore, there are needs to develop the strategy to reduce the risk of advanced prostate cancer.

\subsubsection{Chronic Conditions in Prostate Cancer}

Nearly one-third (30.5\%) of elderly men with prostate cancer had at least one pre-existing chronic conditions. Among those with pre-existing chronic conditions $30 \%$ had 2 or more chronic conditions (5). Among elderly men with prostate cancer, the commonly prevalent chronic condition was diabetes $(13.1 \%)$. Other highly prevalent conditions were: chronic obstructive pulmonary disease (COPD) (9.8\%), depression (8.0\%), congestive heart failure (5.7\%), and coronary artery disease $(2.9 \%)$.

An overwhelming majority of elderly men (84\%) died due to causes other than cancer; in the US, between 1973 and 2008, chronic conditions were the leading causes of death in men with prostate 
cancer (6). Therefore, an understanding the role of particular types of chronic conditions in prostate cancer can provide insight to better manage the chronic conditions as well as prostate cancer.

\subsubsection{Chronic Conditions and Cancer Stage at Diagnosis, Initial Cancer Treatment and Outcomes}

A large body of evidence suggests that men with a greater number of chronic conditions are associated with poor clinical outcomes-bowel, sexual and urinary dysfunction as compared to those with lower numbers of chronic conditions. With regards to cancer stage at diagnosis, the conflicting evidence exist on the presence of chronic conditions and diagnosis of advanced prostate cancer. In terms of the cancer treatment, guidelines recommend no aggressive prostate cancer treatment with RP or RT for individuals with multiple chronic conditions $(4,7)$. However, in the real-world practice settings, mixed evidence exists on receipt of cancer treatment among individuals with chronic conditions and prostate cancer (8-10).

Although elderly men with prostate cancer suffer from different types of chronic conditions, the association between the types of chronic conditions on the cancer stage at diagnosis, initial cancer treatment and clinical outcomes have not been studied. Most of the previous studies focused on the healthcare outcomes as a function of a co-morbidity index, such as Charlson Comorbidity Index (CCI), a numerator for describing weighted/un-weighted number of diseases with little consideration of how specific combinations of conditions affected outcomes among the individuals with prostate cancer. Different types of co-occurring chronic conditions may have a differential impact on cancer stage at the diagnosis, initial cancer treatment and clinical outcomes among men with prostate cancer. Understanding the differential impact is important to develop strategies, which can minimize the negative impact of the chronic conditions on prostate cancer care.

\subsubsection{Case of Metformin: Risk of Advanced Stage of Cancer and Cancer Treatment in Prostate}

\section{$\underline{\text { Cancer and Diabetes }}$}

As stated before, diabetes is highly prevalent among men with prostate cancer (5). Metformin is the first-line of treatment for diabetes with proven safety and efficacy in reducing the risk of cardiovascular disease and improving the blood sugar levels. Recently, due to anti-cancer properties 
of metformin, a significant level of experimental evidence suggests the metformin could be associated with reduced risk of advanced stage of cancer at diagnosis (11) (12-14) (22) (12). In addition, it is also found to be beneficial alone with primary cancer therapy such as RT in reducing the all-cause mortality and cancer-related mortality. Therefore, the use of metformin could influence the initial cancer treatment decision. However, at present, none of the studies have examined the role of metformin on the risk of advanced prostate cancer and influence the initial cancer treatment among elderly men with prostate cancer.

\subsubsection{Impact of Prostate Cancer Diagnosis on Non-cancer Hospitalizations}

As stated above, nearly one third of elderly men with prostate cancer have pre-existing chronic conditions. A diagnosis of incident of prostate cancer may generate the psychological stress and if serious enough could lead to cardiovascular events. The diagnosis of cancer can take the attention away from the management of chronic conditions and all attention may be directed towards cancer care. This may lead to worsening of the pre-existing chronic conditions and can increase the risk of non-cancer hospitalizations. However, none of the studies assessed the impact of diagnosis of prostate cancer on non-cancer hospitalizations among elderly men with incident prostate cancer. An analysis of the extent to which cancer diagnosis affects non-cancer hospitalizations and whether the impact of cancer on non-cancer hospitalizations varies by chronic conditions will inform the payors, program planners and policy-makers and encourage investments in better management of chronic conditions.

\subsection{RATIONALE FOR CLASSIFYING TYPES OF CHRONIC CONDITIONS}

As stated above, men with prostate cancer suffer from multiple conditions. However, operationalizing the measurement of the types of chronic conditions is challenging. For example, if one tries to study chronic conditions and their combinations using only the sixteen chronic conditions captured by the CCI, one can expect 120 two-disease combinations, 560 three-disease combinations, 1,820 four-disease combinations with more than 20 trillion combinations of chronic conditions overall. If one selects chronic conditions based on 30 chronic conditions of Elixhauser index (15), or 53 chronic conditions in elderly men based on Piette et al. framework of chronic conditions 
management (16), one will have even more combinations. While it is possible to use the number of chronic conditions such analyses may not provide any actionable intelligence.

Therefore, in this dissertation, an attempt is made to define clinically meaningful combinations of chronic conditions based on a conceptual framework from the Department of Health and Human Services (DHSS) on research, policy, program, and practice (17). Based on this framework, the following chronic conditions were selected: diabetes, coronary artery disease, cardiac arrhythmia, COPD, asthma, depression, schizophrenia, generalized anxiety disorders, bipolar disorders, and psychosis.

These conditions were grouped based on specific organ domains (22) and synergism in treatment patterns and self-management approaches. For example, management of cardio-metabolic conditions such as diabetes and heart disease have similar treatment patterns and self-management approaches. Thus, the chronic conditions were grouped into three major domains: cardio-metabolic diseases (diabetes, and heart disease), respiratory disease (COPD and asthma), and mental health conditions (depression, bipolar disorders, schizophrenia, psychoses, and anxiety). We classified these domains into eight mutually exclusive categories of chronic conditions: (1) cardio-metabolic conditions only; (2) mental health conditions only; (3) respiratory conditions only; (4) cardiometabolic and mental health conditions, (5) cardio-metabolic and respiratory conditions, (6) respiratory and mental health conditions, (7) all the three conditions, and 8) none of the three types of chronic conditions. However, one should note that such grouping of chronic conditions reflect the presence or absences of multi-organ system conditions, therefore, the types of chronic conditions no longer represent the number of chronic conditions. For example, elderly men grouped in the only cardio-metabolic conditions group may have three chronic conditions-diabetes, coronary artery disease and congestive heart failure, whereas, elderly men with all three types of conditions may also have three chronic conditions such as diabetes, COPD and depression.

\subsection{SPECIFIC AIMS}

The specific aims of the dissertation are as below: 
Specific Aim 1: To examine the associations between the types of pre-existing chronic conditions and cancer stage at diagnosis, initial cancer treatment and clinical outcomes after initial cancer treatment among fee-for-service Medicare beneficiaries with incident prostate cancer.

Objective 1.1: To examine the association between the types of chronic conditions and cancer stage at diagnosis among fee-for-service Medicare beneficiaries with incident prostate cancer.

Hypothesis: Elderly Medicare beneficiaries with only cardio-metabolic, only mental health and only respiratory conditions will be more likely to be diagnosed with advanced prostate cancer as compared to those with all three-cardio-metabolic, mental health, and respiratory conditions.

Objective 1.2: To analyze the association between the types of chronic conditions and cancer treatment (RP, RT, Hormone therapy and No treatment) among fee-for-service Medicare beneficiaries with incident prostate cancer.

Hypothesis: Elderly Medicare beneficiaries with only cardio-metabolic, only mental health and only respiratory conditions will be more likely to receive aggressive treatment (RP/RT) as compared to those with all the three types of chronic conditions.

Objective 1.3: To determine the association between the types of chronic conditions and clinical outcomes (bowel, sexual, urinary dysfunctions) after initial cancer treatment among fee-for-service Medicare beneficiaries with incident prostate cancer.

Hypothesis: Elderly Medicare beneficiaries with only cardio-metabolic, only mental health and only respiratory conditions will be less likely to have bowel, sexual and urinary dysfunctions as compared to elderly men with all the three cardio-metabolic, mental health and respiratory conditions.

Note: In all the analyses that compared across the types of chronic conditions, the reference group was those with all the three types of chronic conditions because the presence of multi-organ system chronic conditions were considered as more serious clinical conditions and expected to be associated with localized stage at diagnosis, no cancer treatment and poor clinical outcomes as compared to other chronic conditions groups.

Specific Aim 2: To examine the relationship between metformin use and cancer stage at diagnosis, and the initial cancer-treatment among elderly (age $\geq 66$ years) fee-for-service Medicare beneficiaries with pre-existing diabetes and incident prostate cancer.

Objective 2.1: To investigate the association between metformin use and cancer stage at diagnosis among elderly fee-for-service Medicare beneficiaries with diabetes and incident prostate cancer.

Hypothesis: Among elderly men with diabetes and incident prostate cancer, metformin users will be less likely to be diagnosed with advanced prostate cancer as compared to non-users.

Objective 2.2: To analyze the association between metformin use and initial cancer treatment among elderly fee-for-service Medicare beneficiaries with diabetes and incident prostate cancer.

Hypothesis: Among elderly men with diabetes and prostate cancer, metformin users will be more likely to receive radiation therapy as compared to non-users; metformin users will be more likely to receive hormone therapy as compared to non-users.

Specific Aim 3: To analyze the impact of cancer diagnosis on the risk of non-cancer hospitalizations and evaluate whether the impact of cancer diagnosis on the risk of non-cancer hospitalizations vary by the types of pre-existing chronic conditions among fee-for-service Medicare beneficiaries with incident prostate cancer.

Objective 3.1: To analyze the association between cancer diagnosis and the risk of non-cancer hospitalizations by comparing the rates of hospitalizations between the pre- and post-cancer periods among elderly men with incident prostate cancer. 
Hypothesis: The unadjusted rates and the adjusted risk of non-cancer hospitalizations will be higher during the post-cancer period as compared to the pre-cancer period.

Objective 3.2: To determine the varying associations between the cancer diagnosis and the risk of non-cancer hospitalizations by the types of chronic conditions among elderly fee-for-service Medicare beneficiaries with incident prostate cancer.

Hypothesis: Elderly Medicare beneficiaries with pre-existing cardio-metabolic conditions will have the greatest increase in the risk of non-cancer hospitalizations (from pre-cancer to post-cancer period) as compared to those with other types of chronic conditions.

\subsection{DATA SOURCES}

The cancer registry data from the Surveillance, Epidemiology and End Results (SEER) program linked with the Medicare administrative claims data were utilized for all the specific aims. These data were augmented with the county-level information contained in the Area Health Resource Files (AHRF).

\subsubsection{Surveillance Epidemiology and End-Results Registry}

The SEER registries are one of the few resources of population-based cancer data in the US Since 1973, SEER registries have been collecting precise and accurate information on cancer stage, treatment, diagnostic procedures, and clinical variables on all newly diagnosed cancer by specific regions under the auspices of the National cancer Institute. The SEER data is released with a lag period of three years. At present, the released SEER data contain a total of 7,397,159 malignant cases of cancers and majorities of these cancer cases are diagnosed among the elderly population aged 65 years and over. The SEER data represent $28 \%$ of the US population from 18 registries or regions of SEER (18).

The Patient Entitlement and Diagnosis Summary File (PEDSF) file is a customized file from the SEER program. It has information on the cancer diagnosis for up to ten cancers, types of cancers, their stages, demographic attributes, marital status, and tumor characteristics at the time of each cancer diagnosis. 


\subsubsection{Medicare}

The Medicare is the US Government mandated insurance program covering $97 \%$ of the US population aged 65 years and older (19). The NCI links the cases of SEER with Medicare cases using an algorithm based on the social security number, last name, first name and birth date of an individual. A total of $93 \%$ percent of men aged 65 years and older in SEER has been linked to Medicare enrollment records (20). More than 95\% of the Medicare beneficiaries receive Part A and B coverage benefits. Medicare Part A benefits cover the use of inpatient care in hospitals or skilled nursing facilities, home health care, and hospice care, whereas Part B benefits cover the use of physician services and outpatient care. However, the Medicare part of data does not capture all the claims for the beneficiaries enrolled under the Health Maintenance Organizations (HMOs) as these organizations do not have a mandatory requirement to submit all their services claims to Center for Medicare and Medicaid (CMS). Therefore, managed care enrollees during the study period were excluded from the study.

\section{SEER-Medicare Linked Data}

A common encrypted identification number is provided for each enrollee so that the PEDSF file can be lined with the Medicare files. Medicare data consisted of Medicare Provider Analysis and Review (MEDPAR) files, the Carrier Claims (old name Physician/Supplier (NCH)), Outpatient (OUTPT), Home Health Agencies (HHA), Hospice, Durable Medical Equipment (DME) and Part D Event (PDE). The MEDPAR file includes Medicare Part A claims records from any short term or long term hospital or skilled nursing facility stay from each calendar year, while the outpatient file has Part B claims for outpatient visits. Each record represents an episode of hospital stay, and has up to 10 diagnoses according to the International Classification of Diseases, Ninth Revision, Clinical Modification (ICD-9-CM), and 10 ICD-9-CM procedures during each stay, date of admission, the date of discharge. The carrier file represents billing records from physicians and non-institutional providers and has procedure codes according to the Healthcare Common Procedure Coding System (HCPCS) and the Common Procedural Terminology, 4th Edition (CPT-4), and ICD-9-CM procedure 
codes with service dates (19). In the current study, we utilized data from the PEDSF file linked with MEDPAR, OUTPT, and NCH files for the years 2000 to 2010.

\subsubsection{Medicare Part-D Data}

Medicare introduced optional/volunteer Part D plans in 2006 that cover prescription drugs benefit through enrollment in a Medicare Advantage Prescription drug Plan (MA-PD) or a stand-alone drug plan (PDP). The plan typically starts with an annual deductible, after which Medicare pays for the prescription drugs up to an initial coverage limit with certain coinsurance associated with those payments. Medicare Part D plans stop payments once individuals reach the annual coverage limit until the cost of prescription drugs reaches to catastrophic limit. The phase between the annual coverage limit and catastrophic coverage is called "donut hole" or "Medication coverage gap" where enrollees have to pay the entire prescription drug costs out-of-pocket (21). Almost half of the Medicare Part A and B enrollee were also enrolled in Part D plans (22). We utilized the data of the Medicare Part D file (PDE) file in addition to PEDSF, MEDPAR, OUTPT and NCH for the years 2007 to 2010 . We examined prostate cancer cases diagnosed between 2008 and 2009 so that we could study their medication status in a year prior to diagnosis of cancer.

\subsubsection{Area Health Resources Files}

The Area Health Resources Files (AHRF) is a nationwide county-level health resource files in the US. The file provides information for about 6,000 county-level variables including healthcare facilities, health professionals, resource scarcity, health status, economic activity, health training programs and socioeconomic characteristics (23). County-level healthcare resources were derived from the Area Health Resource files (AHRF). These included the number of radiation -oncology units, and urology units. The SEER-Medicare files were linked with AHRF using a five-digit Federal Information Processing Standard (FIPS) code at the state and county level (23).

\subsection{CONCEPTUAL FRAMEWORK}

The framework of the expanded Behavioral Model of Health Services Use, widely known as the Andersen Healthcare Utilization and Behavioral Model (ABM) was used to select the independent 
variables for all our specific aims (24). The ABM model and adapted versions of this model have been extensively used in the health services research to study the factors associated with the healthcare service use and healthcare outcomes.

The ABM model states that the use of healthcare services is determined by individual and contextual determinants. These determinants can be categorized into four groups: 1) Predisposing factors: These factors represent the unique feature of individuals that tend to represent more or less use the healthcare services. In the current study, we included age, sex, race/ethnicity, marital status of the individuals as predisposing factors. 2) Enabling factors: These factors serve as conditions that enable individuals to use healthcare services. Based on the model, we considered income, education as well as cancer treatment, and medications as potential enabling factors for the healthcare service. 3) Need factors: These are the factors requiring the need to use healthcare services at the individual level. Based on the definition, we included the presence of chronic conditions and stage of prostate cancer as need factors. 4) External environment: These are the set of factors facilitating the use of healthcare services related to the structure of services in the geographical areas near to the individuals. The presence of healthcare providers in nearby regions such as number of radiation oncologists and urologists in the county of residence of the individuals was included as external environment characteristics. Details on the each type of independent variables for each study aims are provided in Table 1.2. 


\subsection{REFERENCES}

1. American Cancer Society. Cancer Facts \& Figures 2015. Atlanta: American Cancer Society; 2015.

2. Siegel RL, Miller KD, Jemal A. Cancer statistics, 2015. CA: A Cancer Journal for Clinicians. 2015;65(1):529.

3. Edge S, Byrd DR, Compton CC, Greene FL, Trotti A. American Joint Committee on Cancer staging manual, 7th edn . New York : Springer, 2010.

4. Mohler JL, Kantoff PW, Armstrong AJ, Bahnson RR, Cohen M, D'Amico AV, et al. Prostate cancer, version 2.2014. J Natl Compr Canc Netw. 2014 May;12(5):686-718.

5. Edwards BK, Noone AM, Mariotto AB, Simard EP, Boscoe FP, Henley SJ, et al. Annual Report to the Nation on the status of cancer, 1975-2010, featuring prevalence of comorbidity and impact on survival among persons with lung, colorectal, breast, or prostate cancer. Cancer. 2014 May 1;120(9):1290-314.

6. Epstein MM, Edgren G, Rider JR, Mucci LA, Adami HO. Temporal trends in cause of death among Swedish and US men with prostate cancer. J Natl Cancer Inst. 2012 Sep 5;104(17):1335-42.

7. Thompson I, Thrasher JB, Aus G, Burnett AL, Canby-Hagino ED, Cookson MS, et al. Guideline for the management of clinically localized prostate cancer: 2007 update. J Urol. 2007 Jun;177(6):2106-31.

8. Houterman S, Janssen-Heijnen ML, Hendrikx AJ, van den Berg HA, Coebergh JW. Impact of comorbidity on treatment and prognosis of prostate cancer patients: a population-based study. Crit Rev Oncol Hematol. 2006 Apr;58(1):60-7.

9. Nguyen PL, Chen MH, Hoffman KE, Chen RC, Hu JC, Bennett CL, et al. Cardiovascular comorbidity and treatment regret in men with recurrent prostate cancer. BJU Int. 2012 Jul;110(2):201-5.

10. Daskivich TJ, Chamie K, Kwan L, Labo J, Palvolgyi R, Dash A, et al. Overtreatment of men with low-risk prostate cancer and significant comorbidity. Cancer. 2011 May 15;117(10):2058-66.

11. Ashokkumar N, Pari L, Rao C. Effect of N-benzoyl-D-phenylalanine and metformin on insulin receptors in neonatal streptozotocin-induced diabetic rats: studies on insulin binding to erythrocytes. Arch Physiol Biochem. 2006 Jul;112(3):174-81.

12. Hadad SM, Fleming S, Thompson AM. Targeting AMPK: a new therapeutic opportunity in breast cancer. Crit Rev Oncol. 2008;67(1):1-7.

13. Huang X, Wullschleger S, Shpiro N, McGuire V, Sakamoto K, Woods Y, et al. Important role of the LKB1AMPK pathway in suppressing tumorigenesis in PTEN-deficient mice. Biochem J. 2008 Portland Press Limited;412(2):211-21.

14. Bitting RL, Armstrong AJ. Targeting the PI3K/Akt/mTOR pathway in castration-resistant prostate cancer. Endocr Relat Cancer. 2013 May 20;20(3):R83-99.

15. Elixhauser A, Steiner C, Harris DR, Coffey RM. Comorbidity measures for use with administrative data. Med Care. 1998 Jan;36(1):8-27.

16. Piette JD, Kerr EA. The Impact of Comorbid Chronic Conditions on Diabetes Care. Diabetes Care. 2006 March 01;29(3):725-31.

17. Goodman RA, Posner SF, Huang ES, Parekh AK, Koh HK. Defining and measuring chronic conditions: imperatives for research, policy, program, and practice. Prev Chronic Dis. 2013 Apr 25;10:E66. 
18. Surveillance, Epidemiology, and End Results (SEER) Program. SEER*Stat Database: Incidence-SEER 18 Regs Public Use, Nov. 2012 Sub (2000-2010)-Linked to County Attributes-Total US, 1969-2011 Counties. Bethesda, MD: National Cancer Institute, Division of Cancer Control and Population Sciences, Surveillance Research Program, Cancer Statistics Branch; 2013. .

19. Warren JL, Klabunde CN, Schrag D, Bach PB, Riley GF. Overview of the SEER-Medicare data: content, research applications, and generalizability to the United States elderly population. Med Care. 2002 Aug;40(8 Suppl):IV,3-18.

20. SEER-Medicare: How the SEER \& Medicare data are linked? [Internet].; 2013 []. Available from: http://appliedresearch.cancer.gov/seermedicare/aboutdata/.

21. Medicare's New Adventure: The Part D Drug Benefit. The Commonwealth Fund; 2006. [Internet].; 2014 []. Available from: http://www.commonwealthfund.org/ /media/files/publications/fund-

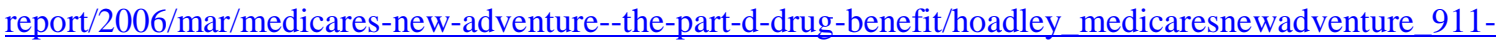
pdf.pdf.

22. Number of SEER-Medicare Patients with Part D Enrollment by Calendar Year Diagnosis Years 1986-2007 [Internet].; 2013 []. Available from: http://appliedresearch.cancer.gov/seermedicare/aboutdata/enrollees.html.

23. Area Resource File: Overview [Internet].; 2014 []. Available from: http://ahrf.hrsa.gov/overview.htm.

24. Andersen RM. Revisiting the behavioral model and access to medical care: does it matter? J Health Soc Behav. 1995 Mar;36(1):1-10. 


\section{Primary tumor $(T)$ - clinical}

TX Primary tumor cannot be assessed

T0 No evidence of primary tumor

T1 Clinically inapparent tumor neither palpable nor visible by imaging

T1a Tumor incidental histological finding in $\leq 5 \%$ of tissue resected

T1b Tumor incidental histological finding in $>5 \%$ of tissue resected

T1c Tumor identified by needle biopsy (e.g. because of elevated PSA)

T2 Tumor confined within prostate

T2a Tumor involves $\leq$ one-half of one lobe

$\mathrm{T} 2 \mathrm{~b}$ Tumor involves > one-half of one lobe but not both lobes

T2c Tumor involves both lobes

T3 Tumor extends through the prostate capsule

T3a Extrascapular extension (unilateral or bilateral)

T3b Tumor invades seminal vesicle(s)

T4 Tumor is fixed or invades adjacent structures other than seminal vesicles such as external sphincter, rectum, bladder, levator muscles, and/or pelvic wall

\section{Regional lymph nodes (N)}

NX Regional lymph nodes were not assessed

N0 No regional lymph node metastasis

N1 Metastases in regional lymph node(s)

\section{Distant metastasis (M)}

M0 No distant metastasis

M1 Distant metastasis

M1a Non-regional lymph node(s)

M1b Bone(s)

M1c Other site(s) with or without bone disease 
Table 1. 2 Details of Variables for Each Study Objectives-classified based on ABM

\begin{tabular}{|c|c|c|c|c|c|}
\hline Objective & $\begin{array}{l}\text { Dependent } \\
\text { Variable (s) }\end{array}$ & Predisposing & Enabling & Need & $\begin{array}{l}\text { External } \\
\text { Environment }\end{array}$ \\
\hline 1.1 & Cancer Stage & $\begin{array}{l}\text { Age, } \\
\text { Race/ethnicity, } \\
\text { Marital status }\end{array}$ & $\begin{array}{l}\text { Income, Education, } \\
\text { Cancer screening, } \\
\text { Primary care visit }\end{array}$ & $\begin{array}{l}\text { Number of } \\
\text { other chronic } \\
\text { conditions }\end{array}$ & $\begin{array}{l}\text { Region, } \\
\text { Urology } \\
\text { Units, } \\
\text { Oncology } \\
\text { units }\end{array}$ \\
\hline 1.2 & $\begin{array}{l}\text { Initial Cancer } \\
\text { Treatment }\end{array}$ & $\begin{array}{l}\text { Age, } \\
\text { Race/ethnicity, } \\
\text { Marital status }\end{array}$ & $\begin{array}{l}\text { Income, Education, } \\
\text { Primary care visit }\end{array}$ & $\begin{array}{l}\text { Number of } \\
\text { other chronic } \\
\text { conditions, } \\
\text { Cancer Stage }\end{array}$ & $\begin{array}{l}\text { Region, } \\
\text { Urology } \\
\text { Units, } \\
\text { Oncology } \\
\text { units }\end{array}$ \\
\hline 1.3 & $\begin{array}{l}\text { Clinical } \\
\text { Outcomes - } \\
\text { Bowl } \\
\text { Dysfunction } \\
\text { Sexual } \\
\text { Dysfunction } \\
\text { Urinary } \\
\text { Dysfunction }\end{array}$ & $\begin{array}{l}\text { Age, } \\
\text { Race/ethnicity, } \\
\text { Marital status }\end{array}$ & $\begin{array}{l}\text { Income, } \\
\text { Education, } \\
\text { Cancer-treatment, } \\
\text { Primary care visit }\end{array}$ & $\begin{array}{l}\text { Number of } \\
\text { other chronic } \\
\text { conditions, } \\
\text { Cancer Stage }\end{array}$ & $\begin{array}{l}\text { Region, } \\
\text { Urology } \\
\text { Units, } \\
\text { Oncology } \\
\text { units }\end{array}$ \\
\hline 2.1 & $\begin{array}{l}\text { Non-cancer } \\
\text { Hospitalizations }\end{array}$ & $\begin{array}{l}\text { Age, } \\
\text { Race/ethnicity, } \\
\text { Marital status }\end{array}$ & $\begin{array}{l}\text { Income, } \\
\text { Education, } \\
\text { Cancer treatment, } \\
\text { Primary care visit }\end{array}$ & $\begin{array}{l}\text { Number of } \\
\text { other chronic } \\
\text { conditions, } \\
\text { Cancer Stage }\end{array}$ & $\begin{array}{l}\text { Region, } \\
\text { Urology } \\
\text { Units, } \\
\text { Oncology } \\
\text { units }\end{array}$ \\
\hline 2.2 & $\begin{array}{l}\text { Non-cancer } \\
\text { Hospitalization }\end{array}$ & $\begin{array}{l}\text { Age, } \\
\text { Race/ethnicity, } \\
\text { Marital status }\end{array}$ & $\begin{array}{l}\text { Income, } \\
\text { Education, } \\
\text { Cancer treatment, } \\
\text { Primary care visit }\end{array}$ & $\begin{array}{l}\text { Number of } \\
\text { other chronic } \\
\text { conditions, } \\
\text { Cancer Stage }\end{array}$ & $\begin{array}{l}\text { Region, } \\
\text { Urology } \\
\text { Units, } \\
\text { Oncology } \\
\text { units }\end{array}$ \\
\hline 3.1 & $\begin{array}{l}\text { Advanced } \\
\text { Prostate Cancer }\end{array}$ & $\begin{array}{l}\text { Age, } \\
\text { Race/ethnicity, } \\
\text { Marital status }\end{array}$ & $\begin{array}{l}\text { Income, } \\
\text { Education, } \\
\text { Cancer Screening, } \\
\text { Primary care visit }\end{array}$ & $\begin{array}{l}\text { Number of } \\
\text { other chronic } \\
\text { conditions, } \\
\text { Cancer Stage }\end{array}$ & $\begin{array}{l}\text { Region, } \\
\text { Urology } \\
\text { Units, } \\
\text { Oncology } \\
\text { units }\end{array}$ \\
\hline 3.2 & $\begin{array}{l}\text { Initial Cancer } \\
\text { Treatment }\end{array}$ & $\begin{array}{l}\text { Age, } \\
\text { Race/ethnicity, } \\
\text { Marital status }\end{array}$ & $\begin{array}{l}\text { Income, } \\
\text { Education, } \\
\text { Cancer-treatment, } \\
\text { Primary care visit }\end{array}$ & $\begin{array}{l}\text { Number of } \\
\text { other chronic } \\
\text { conditions, } \\
\text { Cancer Stage }\end{array}$ & $\begin{array}{l}\text { Region, } \\
\text { Urology } \\
\text { Units, } \\
\text { Oncology } \\
\text { units }\end{array}$ \\
\hline
\end{tabular}

Note: Income and Education were 2000 census-track level data provided in SEER-PEDSF file.

Number of Urology and Radiation-Oncology units are county-level variables derived from Areas Healthcare Resource file (AHRF). The quartiles of these variables were created for data analysis purpose. 


\section{Chapter 2 \\ Types of Chronic Conditions and Cancer Stage at Diagnosis, Initial Cancer Treatment and Clinical Outcomes among Elderly Medicare Beneficiaries with Prostate Cancer}

\subsection{ABSTRACT}

OBJECTIVE: To examine the associations between types of chronic conditions and cancer stage at diagnosis, initial cancer treatment and clinical outcomes among elderly Medicare Beneficiaries with prostate cancer.

METHODS: A population-based observational cohort study was conducted using the Surveillance, Epidemiology and End-Results (SEER)-Medicare linked database. The study population consisted of elderly men ( $\geq 66$ years) with prostate cancer diagnosed between 2002 and $2009(\mathrm{~N}=103,820)$.

Cancer stage at diagnosis (localized versus advanced) was derived using the American Joint Committee on Cancer (AJCC)-Tumor Node Metastasis (TNM) system. The initial cancer treatment was identified during the six months after cancer diagnosis and was categorized in a hierarchical order and consisted of: 1) Radical Prostatectomy (RP); 2) Radiation Therapy (RT); 3) Hormone therapy; and 4) No treatment. Clinical outcomes (bowel dysfunction (yes/no), sexual dysfunction (yes/no) and urinary dysfunction (yes/no)) were measured during one year after the initial cancer treatment.

Chronic conditions were identified during one year before the date of cancer diagnosis and classified as the presence or absence of 1) cardio-metabolic conditions; 2) mental health conditions; and 3) respiratory conditions. These three types of conditions were further classified into the following eight mutually exclusive categories: (1) cardio-metabolic conditions only; (2) mental health conditions only; (3) respiratory conditions only; (4) cardio-metabolic and mental health conditions; (5) cardio-metabolic and respiratory conditions; (6) mental health and respiratory conditions; (7) all three- cardio-metabolic, mental health and respiratory conditions; and 8) none of the three types of conditions. The independent variables were selected based on the Anderson Healthcare Behavior Model (ABM) and consisted of predisposing (age, race/ethnicity, marital status), enabling (income, education, access to care, cancer screening), need (number of other chronic conditions), and externalenvironment (regions, number of radiation-oncology and urology units in a county of residence) factors. 
Significant differences in cancer stage at diagnosis, initial cancer treatment, and clinical outcomes by types of chronic conditions were examined with chi-square tests. Multivariable logistic regressions were used to test the adjusted associations between types of chronic conditions and cancer stage at diagnosis, and clinical outcomes. Multinomial logistic regression was used to test the adjusted associations between types of chronic conditions and receipt of initial cancer treatment.

RESULTS: In our study cohort, $46.2 \%$ did not have any cardio-metabolic, mental health, and respiratory conditions; $35.4 \%$ had only cardio-metabolic conditions; $8 \%$ had both cardio-metabolic and respiratory conditions; $5.2 \%$ had only respiratory conditions; $2.1 \%$ had both cardio-metabolic and mental health conditions; $1.9 \%$ had only mental health conditions; and $0.4 \%$ had both mental health and respiratory conditions.

Cancer Stage at Diagnosis: $94.6 \%$ of the men were diagnosed with localized prostate cancer and $5.4 \%$ were diagnosed with advanced prostate cancer. There was a significant association between types of chronic conditions and cancer stage at diagnosis. The highest percentage $(5.8 \%)$ of advanced prostate cancer diagnosis was observed among elderly men with none of the three types of chronic conditions. After controlling for predisposing, enabling, need and external environment factors, elderly men with none of the three types of chronic conditions were $44 \%$ more likely to be diagnosed with advanced prostate cancer as compared to men with all the three types of chronic conditions (Adjusted Odds Ratio $(\mathrm{AOR})=1.44 ; 95 \%$ Confidence Interval $(\mathrm{CI}): 1.03,2.00)$.

Initial Cancer Treatment: In our study cohort, $19.7 \%$ did not receive any cancer treatment; $46.9 \%$ received RT; $22 \%$ received RP; and $11.4 \%$ received hormone therapy. The highest percentage of no prostate cancer treatment (24.4\%) was observed among men with all the three types of chronic conditions and lowest percentage of no prostate cancer treatment was observed among men with only respiratory conditions (18.3\%). In multinomial logistic regression analysis, elderly men with none of the three types of chronic conditions (AOR $=1.32 ; 95 \%$ CI: $1.07,1.61)$ or only one type of chronic physical condition - only cardio-metabolic conditions (AOR $=1.25 ; 95 \% \mathrm{CI}: 1.02,1.53)$ or only respiratory conditions $(\mathrm{AOR}=1.36 ; 95 \% \mathrm{CI}: 1.09,1.69)$ were more likely to receive $\mathrm{RP}$ as compared to those with all the three types of chronic conditions. These groups were also more likely to receive 
RT. Additionally, elderly men with cardio-metabolic and mental health conditions or respiratory conditions were more likely to receive RT. Those with only mental health conditions were as likely as those with all the three types of chronic conditions to receive initial cancer treatment. There were no statistically significant relationships between types of chronic conditions and receipt of only hormone therapy among elderly men with prostate cancer.

Clinical Outcomes: Overall, $14.5 \%$ elderly men had bowel dysfunction; $16.1 \%$ had sexual dysfunction; and 35.0\% had urinary dysfunction. In multivariable logistic regression, elderly men with none of the three types of conditions $(\mathrm{AOR}=1.33$; 95\% CI: $1.09,1.63)$ or only respiratory (AOR $=1.26 ; 95 \%$ CI: $1.02,1.56)$ or only mental health conditions (AOR $=1.32 ; 95 \%$ CI: $1.05,1.67)$ were more likely to have sexual dysfunction as compared to those with all the three types of chronic conditions. There were no consistent patterns with regard to types of chronic conditions and clinical outcomes.

CONCLUSIONS: Elderly men with none of the three types of chronic conditions were more likely to be diagnosed with advanced prostate cancer. All chronic condition categories (except for only mental health conditions and both mental health and respiratory conditions) were associated with an increased likelihood of the initial cancer treatment with either RP or RT as compared to all the three types of chronic conditions. Varying associations between types of chronic conditions and clinical outcomes were found.

IMPLICATIONS: Among elderly men without cardio-metabolic, respiratory and mental health conditions, strategies to reduce the risk of advanced prostate cancer are needed. Although there is no evidence that RP or RT can be beneficial in long-term for elderly men with prostate cancer and preexisting chronic conditions, an overwhelming majority of men with chronic conditions received prostate cancer treatment. Men with prostate cancer and some types of chronic conditions may need heightened surveillance, monitoring and therapies to reduce the risk of sexual dysfunction after the initial cancer treatment. 


\subsection{INTRODUCTION}

Chronic conditions are highly prevalent among elderly men diagnosed with prostate cancer in the United States (US). Nearly one-third (31\%) of elderly men with incident prostate cancer have at least one pre-existing chronic condition out of sixteen chronic conditions included in the Charlson Comorbidity Index (CCI) (1). The presence of pre-existing chronic conditions can affect the cancer stage at diagnosis (2-7), receipt of the initial cancer treatment (8-12) and clinical outcomes such as bowel, sexual, and urinary dysfunctions (13-15) among elderly men with prostate cancer. With regards to types of chronic conditions, cardio-metabolic conditions (diabetes and cardiovascular diseases) are the most common pre-existing chronic conditions (18.1\%), followed by respiratory conditions (example - chronic obstructive pulmonary disease (COPD) (9.8\%), (1) and mental health conditions (example - depression) (9\%) among elderly men with prostate cancer (16). Therefore, the presence of such different types of chronic conditions can differently influence the development of cancer, cancer treatment and outcomes among elderly men with prostate cancer.

\subsubsection{Pre-existing Chronic Conditions and Cancer Stage at Diagnosis}

More than two-third cases of prostate cancer are diagnosed at localized stages ( $\leq \mathrm{T} 2$ stage), in which tumors are confined to the prostate gland, whereas less than one third of cases of prostate cancer are diagnosed at advanced stages where tumors have spread to nearby tissue, bone or organs. The diagnosis of prostate cancer at localized stage is desirable as compared to diagnosis of advanced stage due to an excellent survival profile with localized stage even without active cancer treatment. The presence of chronic conditions can affect the cancer stage at diagnosis for many reasons. There are conflicting theories on the relationship between the presence of chronic conditions and risk of advanced prostate cancer at diagnosis. One of these theories hypothesizes that individuals with chronic conditions may be less likely to be diagnosed at an advanced prostate cancer because of increased contact with the healthcare system for care of their chronic conditions (17). Conversely, the theory on competing demand postulates that the care of chronic conditions may take the attention away from early symptoms of cancer and that individuals with chronic conditions may be more likely to be diagnosed with an advanced prostate cancer. The role of competing demands for care of 
chronic conditions on advanced prostate cancer at diagnosis has been documented. For example, men with prostate cancer and pre-existing congestive heart failure were more likely to be diagnosed with advanced prostate cancer as compared to those without congestive heart failure (2).

Further, the association between pre-existing chronic conditions and cancer stage at diagnosis may differ by types of chronic conditions, due to the differential patho-physiological pathways of underlying diseases and prostate cancer. For example, the presence of cardiovascular disease may increase the risk of prostate cancer as it shares common risk factors for the development of aggressive prostate cancer, such as the presence of high cholesterol level and obesity $(18,19)$. Few studies have investigated the relationship between specific types of chronic conditions and the cancer stage at diagnosis. Findings from these studies suggest that the association between pre-existing chronic conditions and cancer stage at diagnosis depends on the types of chronic conditions. For example, three studies in the US using the population-level data found that the presence of diabetes was associated with an increase in the risk of advanced stage or grade of prostate cancer at diagnosis (2022). With respect to the mental health conditions, a retrospective study found that elderly men with depression were not more likely to be diagnosed with higher grade of prostate cancer as compared to those without depression (9). While these studies have highlighted the relationship between specific chronic conditions and cancer stage at diagnosis, many of these studies are outdated, singleinstitutional database studies and suffer from the risk of detection bias because screening for prostate cancer was not included as one of the independent variables (4-6). Furthermore, none of these studies examined the association between types of chronic conditions combinations and cancer stage at diagnosis among elderly men. Understanding the association between types of chronic conditions and cancer stage at diagnosis is critical for patient-centered care and individualized treatment (23).

\subsubsection{Pre-existing Chronic Conditions and Prostate Cancer Treatment}

As elderly men with prostate cancer are at greater risk for mortality due to non-cancer conditions as compared to risk for mortality due to cancer, the practitioners determine the course of cancer treatment after taking into account the presence of pre-existing chronic conditions in addition to the cancer stage at diagnosis and life-expectancy. The clinical practice guidelines of the American 
Urology Association (AUA) (24) and the National Cancer Comprehensive Network (NCCN) (25) recommend against the use of aggressive treatment such as RP or RT among men who have serious chronic conditions.

\subsubsection{Number or Weighted Score of Chronic Conditions}

Some studies have investigated the association between chronic conditions and the use of aggressive cancer treatment such as RP or RT among elderly men with prostate cancer. Using the Charlson Comorbidity Index (CCI) score to summarize the impact of chronic conditions, prior studies have found varying associations between the presence of chronic conditions and receipt of a particular prostate cancer treatment. Two retrospective studies reported that men with prostate cancer and greater CCI scores were less likely to receive RP/RT as compared to those with lower CCI scores (10, 26). Conversely, two studies reported that men with prostate cancer having greater CCI scores were more likely to receive RP/RT as compared to those with lower CCI scores (11) (12). Finding from these studies suggests that weighted summary scores such as CCI may not be useful in determining the course of prostate cancer treatment. Therefore, it is important to analyze the relationship between specific types of chronic conditions and receipt of a particular cancer treatment among men with prostate cancer.

\subsubsection{Presence of a Specific Chronic Condition}

Two previous studies have examined the association between specific chronic conditions and prostate cancer treatment. Hall et al. found that men with congestive heart failure were less likely to receive surgical therapy as compared to those without congestive heart failure (27). Using the SEERMedicare linked data, Prasad et al. reported that men with depression were less likely to receive aggressive therapy such as RP or RT as compared to those without depression (6). While these studies suggest the presence of single chronic condition such as congestive heart failure or depression would have negative impact on the receipt of RP/RT among men with prostate cancer, these studies did not account for the presence of other co-existing chronic conditions. However, in the real-world practice settings, elderly men have multiple chronic conditions which may determine the course of cancer treatment. To the best of our knowledge, studies analyzing the associations between types of 
chronic conditions and their combinations and receipt of initial treatment for prostate cancer are nonexistent.

\subsubsection{Preexisting Chronic Conditions and Clinical outcomes}

\subsubsection{Number or Weighted Score of Chronic Conditions:}

Among elderly men with prostate cancer, pre-existing chronic conditions can affect clinical outcomes such as bowel, urinary and sexual dysfunctions. A handful of real-world observational studies have examined the relationship between CCI and the risk of urinary, bowel and sexual dysfunctions following the initial cancer treatment among elderly men with prostate cancer. These studies reported that elderly men with higher CCI scores were more likely to have bowel, and urinary dysfunctions $(13-15,28)$ as compared to those with lower CCI scores. Using the SEER-Medicare linked data, one study found that CCI scores were not associated with sexual dysfunction (29). Again, the weighted summary scores such as CCI may not be useful in determining strategies for reducing the risk of these dysfunctions after the initial cancer treatment. Therefore, it is important to analyze the relationship between specific types of chronic conditions and clinical outcomes.

\subsubsection{Presence of a Specific Chronic Condition}

A single-institutional study by Hamstra et al. analyzed relationship between the presence of myocardial infarction and the risk of bowel dysfunction among men with prostate cancer. The authors found that men with myocardial infarction were more likely to have bowel dysfunction during the three years after the initial cancer treatment as compared to those without myocardial infarction (13). To the best of our knowledge, none of the population-based studies examined the association between types of chronic conditions and the clinical outcomes such as bowel, urinary and sexual dysfunction among elderly men with prostate cancer.

Based on the literature review till date, it can be concluded that knowledge gaps exist in how specific combinations of conditions affect the cancer stage at diagnosis, the initial cancer treatment and the clinical outcomes among elderly men with incident prostate cancer. Examining the associations between combinations of chronic conditions and cancer stage at diagnosis, initial cancer 
treatment and clinical outcomes are important because such analyses can inform the practitioners on the management of cancer care in the presence of chronic conditions. Therefore, the primary objectives of the current study are to examine the associations between the types of pre-existing chronic conditions and cancer stage at diagnosis, initial cancer treatment, and clinical outcomes after the initial cancer treatment among fee-for-service Medicare beneficiaries with incident prostate cancer.

\subsection{CONCEPTUAL FRAMEWORK}

To accomplish the objectives, the current study adapted the Andersen Behavioral Model, which is described in chapter 1 . The selected predisposing, enabling, need and external environmentrelated factors for the current study are depicted in Figure 2.1 .

Figure 2.1 Study Theoretical Framework: Adaptation of Andersen Behavioral Model for Aim 1

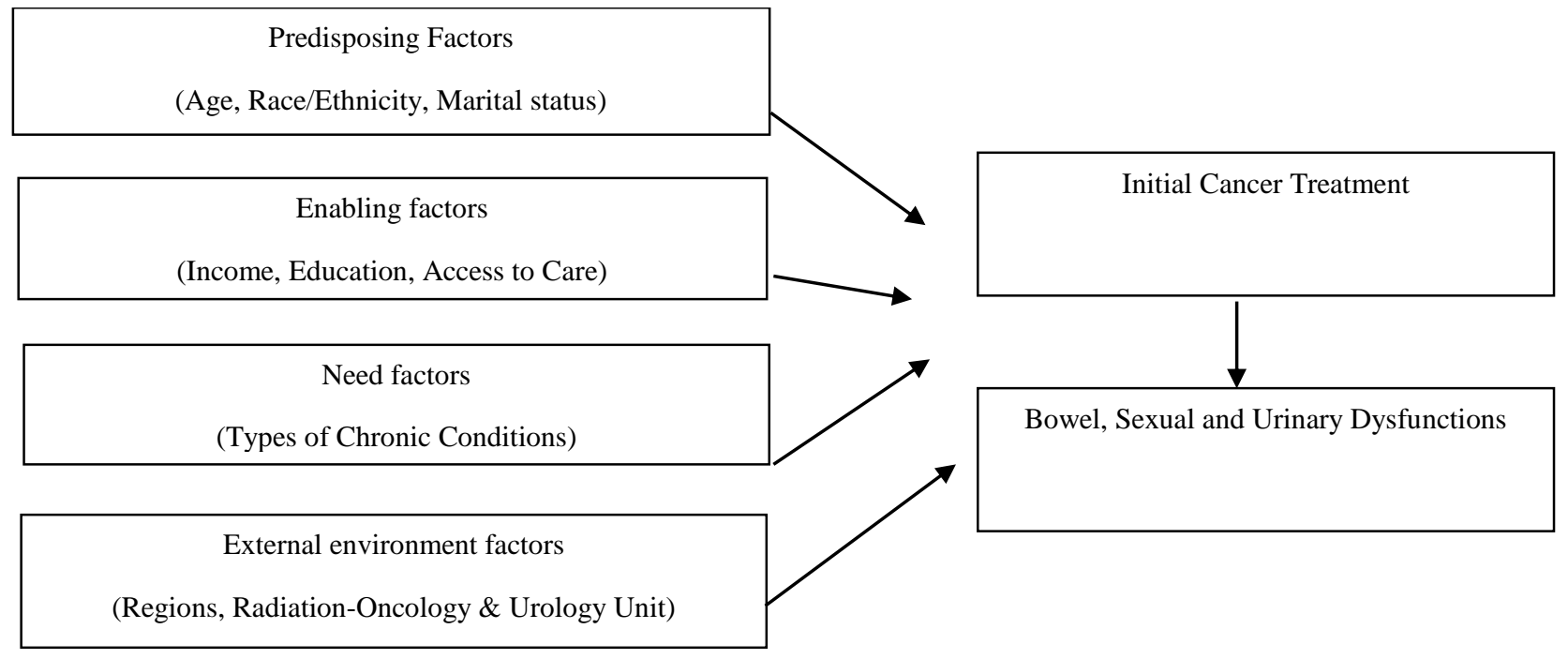

\subsection{METHOD}

\subsubsection{Study Design}

This study adopted a retrospective cohort design with a baseline and follow-up period. The date of diagnosis of prostate cancer was considered as the index date. The baseline period was defined as one year before the index date. All independent variables were identified during the baseline period. For objective 1.2, the follow-up period consisted of 6 months after prostate cancer diagnosis. Initial prostate cancer treatment was measured during this period. For objective 1.3, the clinical 
outcomes were identified during a 12 months after the end of initial prostate cancer treatment (i.e. end of 6-months) as depicted in the Figure 2.2.

Figure 2.2 Schematic diagram of study design for Aim 1

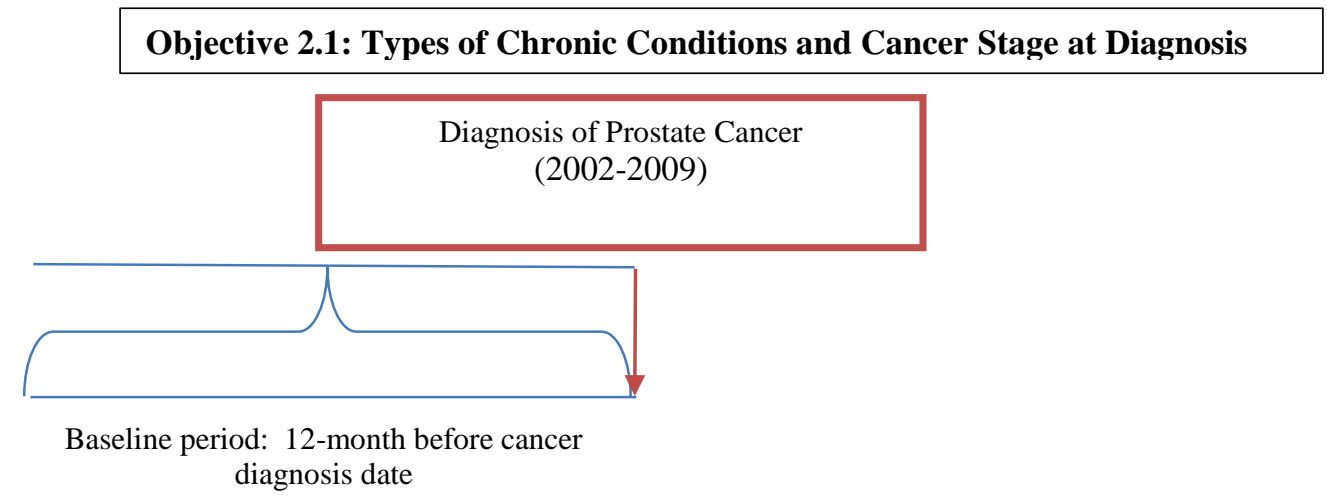

Objective 2.2: Types of Chronic Conditions and Cancer Treatment

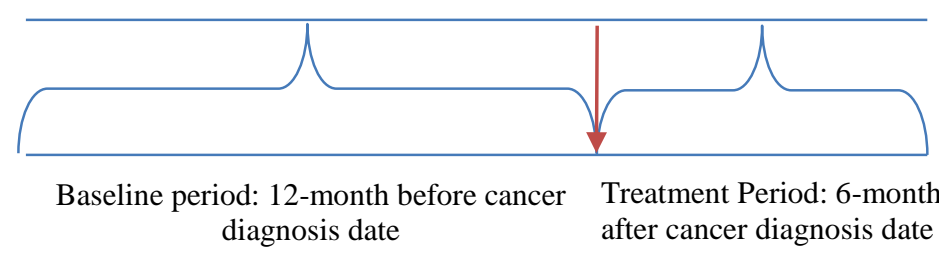

Objective 2.3: Types of Chronic Conditions and Clinical Outcomes

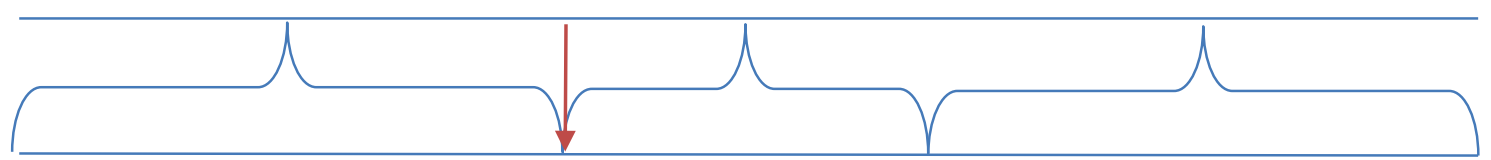

Baseline period: 12-month Treatment Period: 6 month 2001- 2009

Follow-up Period: 12-month after Initial Cancer Treatment

\subsubsection{Data Sources}

The current study utilized the SEER-Medicare linked database. The SEER part of the data comprised of cancer cases diagnosed with prostate cancer between January $1^{\text {st }} 2002$ and December $31^{\text {st }} 2009$ and linked with Medicare claims of those individuals for the years January $1^{\text {st }} 2000$ and December $31^{\text {st }} 2010$. The detailed description of the data sources are provided in Chapter 1 . 


\subsubsection{Study Cohort}

The study cohort was based on 358,439 men with an incident diagnosis of prostate cancer. Elderly men with multiple cancers $(\mathrm{N}=65,794)$, diagnosed with prostate cancer at the time of autopsy or on the death certificate $(\mathrm{N}=2,944)$, younger than 66 years of age $(\mathrm{N}=97,159)$, died during the observation period ( $\mathrm{N}=41,205)$, did not have continuous fee-for-service enrollment in Medicare Part A and $\mathrm{B}$ during the observation period $(\mathrm{N}=70,559)$, and missing information on cancer stage at diagnosis, race, income, education or county of residence $(\mathrm{N}=5,390)$ were excluded. Thus, the final cohort consisted of 103,820 elderly men with incident prostate cancer. Appendix 2.1 depicts the analytical population selection process.

\subsubsection{Key Dependent Variables}

\subsubsection{Prostate Cancer Stage at Diagnosis (Localized and Advanced Stage)}

We utilized the American Joint Committee on Cancer (AJCC) Tumor-Node-Metastases (TNM) classifications for staging of prostate cancer. Based on the TNM systems, we classified cases with into two categories: localized and advanced prostate cancer at diagnosis. Localized prostate cancer was classified as the cancer with $\mathrm{T} 1$ to $\mathrm{T} 2$ clinical stage with no regional lymph node (NX-N0) or distant metastasis (M0) (24). Advanced prostate cancer was classified as the cancer with T3 or T4 clinical stage with or without regional lymph node (N1) or distant metastasis (M1).

\subsubsection{Initial Cancer Treatment}

The receipt of initial prostate cancer treatment as either receipt of radiation or surgical therapy or hormonal therapy was identified using inpatient, outpatient and carrier files with appropriate ICD-9 CM diagnostic and procedure codes, CPT or HCPCS codes or Revenue Center code during the six months after the index date $(30,31),(32)$. Appendix 2.2 comprises the details of the codes used to identify the initial cancer treatment in the current study.

We classified elderly men with the initial cancer treatment receipt into four hierarchical groups: 1) those who received RP alone or along with any RT or hormone therapy; 2) those who received RT alone or along with hormone therapy; 3) those who only received hormone therapy; and 
3) no-treatment group as those who did not receive RP or RT or hormone therapy during six-month after the diagnosis of prostate cancer. Such grouping of the initial cancer treatment hierarchical preference for particular cancer treatments among men with prostate cancer.

\subsubsection{Clinical Outcomes-Urinary, bowel, and sexual dysfunction}

Clinical outcomes consisted of bowel, sexual, and urinary dysfunction during one-year after the initial cancer treatment. The bowel, sexual and urinary dysfunction were identified using ICD-9 CM codes and HCPCS codes from the inpatient, outpatient and carrier claims files during one year after the initial cancer treatment. Codes used to define urinary, bowel, and sexual dysfunction are listed in Appendix 2.3. Bowel dysfunction included the presence of rectal hemorrhage, ulcers, fistulas, hyperbaric oxygen therapy and rectal repairs; and sexual dysfunction included the presence of impotence, penile prostheses, and intra-cavernosal injections; (33), whereas, urinary dysfunction included the presence of any of the following conditions: incontinence, obstruction, irradiation cystitis, bladder hemorrhage, and urinary fistulas (34).

\subsubsection{Key Independent Variable}

\subsubsection{Types of pre-existing chronic conditions}

Chronic conditions were identified using one inpatient or two outpatient claims with ICD-9 $\mathrm{CM}$ diagnostic codes and procedure codes during the year before diagnosis of prostate cancer (Appendix 2.4). To define clinically meaningful combinations of chronic conditions, we used several criteria. These included: high prevalence, specific organ domains, common pathophysiology and synergistic management of different chronic conditions (35). Based on prior literature, as well as the conceptual model of chronic condition measurement, we selected 12 chronic conditions. These 12 chronic conditions were among the 20 conditions selected by the US Department of Health and Human Services (DHS) for research, policy, program and practice (36). These 12 conditions were grouped into 3 broad categories using specific organ domains, common pathophysiology and synergistic management. They were: cardio-metabolic conditions (diabetes, coronary artery disease, congestive heart failure, and cardiac arrhythmia), mental health conditions (anxiety, bipolar disorders, 
depression, schizophrenia, post-traumatic stress disorders, and psychosis) and respiratory conditions (COPD and asthma). Such type of classifications have been used in many studies (37-39).

Furthermore, we classified these three types of chronic conditions into eight mutual categories: (1) cardio-metabolic conditions only (2) mental health conditions only; (3) respiratory conditions only (4) cardio-metabolic and mental health conditions; (5) cardio-metabolic and respiratory conditions; (6) mental health and respiratory conditions (7) all three -cardio-metabolic, mental health and respiratory conditions; and 8) none of the three types of conditions.

\subsubsection{Other Independent Variables}

\subsubsection{Predisposing Characteristics}

In the current study, the demographic factors such as age, race/ethnicity, and marital status were included as the predisposing characteristics. Age at the time of diagnosis was identified from the PEDSF file and was categorized into two groups (66 to 74 years, 75 and above years). Race/ Ethnicity and marital status were also derived from the PEDSF file. Race/Ethnicity was categorized into four groups: White, African American, Hispanic, and other. Marital status was categorized into four groups: Married, Divorced/Separated, Unmarried, and others.

\subsubsection{Enabling Characteristics}

Enabling factors were income, education, access to care and prostate-cancer screening. The PEDSF file contains census-tract level information on income and education variables. Access to care was defined using a proxy measure in the absence of any direct measures for the same. The proxy for access to care was measured using visits to a primary care provider during the baseline period (40). The HCPCS codes of 84152, 84154, 84153, G0103 represented prostate-specific-antigen (PSA) test, and these codes were used to identify prostate-cancer screening during the bassline period.

\subsubsection{Need Characteristics}

During the baseline period, a very small percentage of men $(<5 \%)$ had at least one of the other forty-two conditions, which were not captured by the cardio-metabolic, mental health, and respiratory conditions. These forty-two other conditions were derived using the list of chronic 
conditions from published literature (41). The total number of other chronic conditions were calculated and categorized into two groups as the presence of either " $0-1$ condition" or "two or more conditions".

\subsubsection{External environment characteristics}

External environment characteristics comprised of individual's-resident level county, region, county-level radiation oncology units and urology units. The 18 regions of SEER were categorized into four groups:1) Northeast-which included two registries of Connecticut, and New Jersey;2) South- which included five registries from Atlanta, Greater Georgia, Kentucky, Louisiana, and Rural Georgia,; 3) North-Central which included two registries from Detroit and Iowa; and 4) West which included registries from Arizona, Alaska, Greater California, Cherokee Nation, Hawaii, Los Angeles, New Mexico, San Francisco-Oakland, San Jose-Monterey, Seattle-Puget Sound, and Utah (42).

County-level healthcare resources were derived from the AHRF. The AHRF is a nationwide county-level health resource files in the US. The file provides information for about 6,000 countylevel variables including healthcare facilities, health professionals, resource scarcity, health status, economic activity, health training programs and socioeconomic characteristics (42). The number of radiation -oncology units, and urology units in counties were used to present county-level healthcare resources. SEER-Medicare files were matched with the AHRF using FIPS state and county codes (42). For the purposes of our analyses, the quartiles of radiation-oncology units and urology units at the county level were calculated. In addition, the year of diagnosis was also considered as an external factor.

\subsection{STATISTICAL ANALYSES}

The significant differences in predisposing, enabling, need, and external environment characteristics by types of pre-existing chronic conditions among the men with incident prostate cancer were tested using chi-square tests. Multivariable binary logistic regressions were used to test the association between types of chronic conditions and advanced prostate cancer diagnosis after controlling for the predisposing, enabling, need, and external environment characteristics. 
Multinomial logistic regressions were performed to test the association between types of chronic conditions and cancer treatment receipt (RP, RT, hormone therapy only) after controlling predisposing, enabling, need and external environment characteristics. In this regression, cancer stage was additionally included as a need factor. Separate multivariable binary logistic regressions were used to test the associations between types of chronic conditions and clinical outcomes (urinary, bowel or sexual dysfunction) after adjusting for predisposing, enabling including the initial cancer treatment, need, and external environment characteristics. In these regressions, cancer stage at diagnosis was included as a need factor and cancer treatment was included as an enabling factor. All statistical analyses were carried out using Statistical Analysis System (SAS) version 9.4 (SAS Institute Inc., Cary, NC).

\subsection{RESULTS}

\subsubsection{Study Population}

Table 2.1 summarizes the characteristics of the study cohort $(\mathrm{N}=103,820)$. A majority of men were diagnosed with prostate cancer between age 66 and 74 years (59.7\%). The study cohort was primarily white (81.7\%) and married (67.1\%). Nearly three-quarters of men had at least one primary care visit $(73.8 \%)$ and had a screening of PSA (81.6\%) during the baseline period.

\subsubsection{Study Population by Types of Chronic Conditions}

Table 2.2 reports the characteristics of the study population by types of chronic conditions. Overall, $53.8 \%$ of the study population had either cardio-metabolic, mental health, or respiratory conditions during the year before the prostate cancer diagnosis and $46.2 \%$ had none of these conditions. The majority of men had only cardio-metabolic conditions (35.4\%), followed by men with both cardio-metabolic and respiratory conditions (8\%), only respiratory conditions (5.2\%), cardiometabolic and mental health conditions (2.1\%), only mental health conditions (1.9\%), and mental health conditions and respiratory conditions $(0.4 \%)$. Significant differences in the predisposing, enabling, need and external environment factors by types of chronic conditions were observed among elderly men with incident prostate cancer. 


\subsubsection{Types of Chronic Conditions and Cancer Stage at Diagnosis}

Table 2.3 describes the relationship between the types of chronic conditions and cancer stage at diagnosis. Overall, $94.6 \%$ of the study cohort were diagnosed with localized prostate cancer and $5.4 \%$ were diagnosed with advanced prostate cancer. Significant relationships between types of chronic conditions and cancer stage at diagnosis were observed. As compared to men with all the three types of chronic conditions (4.5\%), a lower percentage of men with cardio-metabolic and respiratory conditions were diagnosed (3.8\%) with advanced prostate cancer.

Table 2.3 also reports the adjusted odds ratios (AOR) and 95\% Confidence Intervals (CI) from the logistic regression on cancer stage at diagnosis. After adjusting for predisposing, enabling, need and external environment factors among elderly men with prostate cancer, elderly men with none of the three conditions were $44 \%$ more likely to be diagnosed with advanced prostate cancer as compared to those with all the three types of chronic conditions $(\mathrm{aOR}=1.44,95 \% \mathrm{CI}=1.03,2.00)$.

\subsubsection{Types of Chronic Conditions and Initial Cancer Treatment Type}

Tables 2.4 displays the rates of initial cancer treatment by types of chronic conditions. Overall, $80.3 \%$ of the study population received either RP/RT or hormone therapy during the six months after the cancer diagnosis: 22 received RP; $46.9 \%$ received RT; $11.4 \%$ received hormone therapy, and $19.7 \%$ received no treatment for cancer. The highest rate of RP was observed among those with only mental health conditions (24.4\%), whereas, the highest rate of RT was observed among elderly men with cardio-metabolic conditions (49\%). The highest rates of only hormone therapy $(16.2 \%)$ or no treatment $(24.4 \%)$ were observed among elderly men with prostate cancer with all the three types of chronic conditions (59.4\%).

The bottom panel of Table 2.4 displays the AORs and 95\% CIs from the multinomial logistic regression on initial cancer treatment categories. After adjusting for predisposing, enabling, need factors including cancer stage, and external-environment factors, elderly men with none of the three types of chronic conditions $(\mathrm{AOR}=1.32,95 \% \mathrm{CI}: 1.07,1.61)$ or only cardio-metabolic conditions $(\mathrm{AOR}=1.25 ; 95 \% \mathrm{CI}: 1.02,1.53)$ or only respiratory conditions $(\mathrm{AOR}=1.36 ; 95 \% \mathrm{CI}: 1.09,1.69)$ 
were more likely to receive RP as compared to those with all the three types of chronic conditions. These groups were also more likely to receive RT. Additionally, elderly men with cardio-metabolic and mental health conditions (AOR: $1.2795 \%$ CI: 1.04, 1.57) or respiratory conditions (AOR: 1.49, 95\% CI: $1.24,1.79)$ were more likely to receive RT. Those with only mental health conditions were as likely as those with all the three types of chronic conditions to receive initial cancer treatment. There were no statistically significant relationships between types of chronic conditions and receipt of only hormone therapy among elderly men with prostate cancer.

\subsubsection{Types of Chronic Conditions and Clinical Outcomes}

Table 2.5 reports rates of clinical outcomes by the types of chronic conditions. Overall, $14.5 \%$ elderly men had any bowel dysfunction; $16.1 \%$ had any sexual dysfunction and $35.0 \%$ had any urinary dysfunction. Elderly men with prostate cancer and all the three types of chronic conditions had the highest rate of bowel dysfunction (20.5\%) whereas, men with mental health conditions had the highest rate of sexual dysfunction (17.4\%) and men with cardio-metabolic and mental health conditions had the highest rates of urinary dysfunction (44.3\%).

In adjusted analysis, as compared to men with all the three types of chronic conditions, elderly men with none of the three types of conditions (AOR $=1.33$; 95\% CI: $1.09,1.63$ ) or only respiratory $(\mathrm{AOR}=1.26 ; 95 \% \mathrm{CI}: 1.02,1.56)$ or only mental health conditions $(\mathrm{AOR}=1.32 ; 95 \% \mathrm{CI}$ : $1.05,1.67)$ were more likely to have sexual dysfunction. There were no consistent patterns with regard to the types of chronic conditions and bowel dysfunction or urinary dysfunction.

\subsection{DISCUSSION}

One in two elderly men (54\%) with incident prostate cancer had pre-existing cardiometabolic, mental health, and respiratory conditions. The commonly prevalent types of chronic conditions were: cardio-metabolic and respiratory chronic conditions (15\%). These findings suggest that elderly men with prostate cancer have significant disease burden prior to the diagnosis of prostate cancer. 


\subsubsection{Types of Chronic Conditions and Cancer Stage at Diagnosis}

In our study cohort, 1 in 10 elderly men with prostate cancer were diagnosed with advanced prostate cancer. Our study estimates are consistent with published literature on rates of advanced prostate cancer $(6,9,43)$. A statistically significant association was observed between the absence of any three types of chronic conditions and diagnosis with advanced prostate cancer. Closer examination of our results suggested that a significantly lower proportion of elderly men with none of three types of chronic conditions had a PSA screening and visit to a primary care physician in the year before the diagnosis of cancer as compared to those with any other types of chronic conditions. Taken together these findings confirm that contact with healthcare system may improve screening for prostate cancer (44). Elderly men with cardio-metabolic, respiratory and mental health conditions are more likely to use healthcare before diagnosis of cancer as compared to those without these chronic conditions leading to higher likelihood of prostate cancer screening. Therefore, our study findings suggest a strong need for the screening those with none of the three common types of chronic conditions.

\subsubsection{Types of Chronic Conditions and Initial Cancer Treatment}

Nearly 4 in 5 elderly men with incident prostate cancer received initial cancer treatment. Our findings are consistent with published studies $(8,27)$. Elderly men with none of the three types of chronic conditions were more likely to receive initial cancer treatment compared to those with all the three types of chronic conditions. Clinical guidelines encourage aggressive treatment with RP or RT for men without pre-existing chronic conditions because these men are considered relatively healthy and therefore may have long-term survival benefit and a better prognosis from initial cancer treatment.

Despite the availability of accurate estimates of survival based on age, CCI score and cancer stage $(8,9,11)$, in the current study, elderly men with prostate cancer and some types of chronic conditions were more likely to be treated for cancer as compared to those with all the three types of chronic conditions. These findings suggest that many elderly men with chronic conditions may have been treated aggressively even though such treatment may not be provide long-term survival benefit 
(45) and may lead to adverse health consequences (46). The current guidelines for prostate cancer treatment recommend "no aggressive treatment should be an option for those with serious chronic conditions". However, these guidelines did not explicitly define the term "serious chronic conditions". Therefore, the providers may not consider the presence of some types of chronic conditions serious enough and may have treated the cancer aggressively with RP/RT. Our study reinforces the need for clinical guidelines that explicitly incorporate the types of chronic conditions along with age and life-expectance to recommend cancer treatment options for elderly men with prostate cancer.

Even after controlling for cancer stage and other factors, men with prostate cancer and only cardio-metabolic conditions were more likely to receive RP/RT as the initial cancer treatment as compared to those with all the three types of chronic conditions. Prior research has shown that elderly men with cardio-metabolic conditions may not benefit from cancer treatment as these men are at high risk for non-cancer related mortality $(8,45)$. There is also evidence of the increased risk for adverse clinical outcomes such as poor wound healing, risk of perioperative complications due to cancer treatment $(47,48)$ and treatment regret $(46)$ among elderly men with prostate cancer and pre-existing cardio-metabolic conditions. Our study findings highlight the need to increase the physician and patient awareness of adverse risks associated with active treatment among elderly men with prostate cancer and cardio-metabolic conditions.

Another noteworthy finding from this study is that the elderly men with prostate cancer and only mental health conditions and both mental health conditions and respiratory conditions did not have significant differences between the receipt of RP/RT treatment. These findings are not consistent with a published study in which elderly men with prostate cancer and mental health conditions were less likely to receive aggressive treatment compared to elderly men without mental health conditions. The difference in findings could be explained by lack of adjustments for types of chronic conditions in the current study (6). 


\subsubsection{Types of Chronic Conditions and Clinical Outcomes}

Consistent with previous studies (13-15), poor clinical outcomes were common among elderly men with prostate cancer during the year after the initial cancer treatment. The current study is the first of its kind to examine the relationships between types of chronic conditions and clinical outcomes. Elderly men with only mental health conditions and only respiratory conditions were more likely to have sexual dysfunction as compared to men with all the three types of chronic conditions. Similar to our study finding, Punnen et al. found that the presence of depression was associated with sexual dysfunction even after controlling for the primary treatment choice among men with prostate cancer in a prospective cohort study (49). Therefore, our study findings reinforce the need to actively monitor men with prostate cancer and only mental conditions or only respiratory conditions for reducing the risk of sexual dysfunction.

Elderly men with prostate cancer and some types of chronic conditions were less likely to experience bowel and urinary dysfunction, after controlling for the initial cancer treatment and other factors. For example, as compared to those with all the three types of chronic conditions, elderly men with prostate cancer and only cardio-metabolic or only respiratory conditions were less likely to have bowel and urinary dysfunction. The reasons for why these particular combinations are associated with lower risk of bowel and urinary dysfunction are unknown. Future research needs to explore the possible patho-physiological link between types of chronic conditions and bowel and urinary dysfunction.

Finally, our study findings have to be interpreted in the context of strengths and limitations. Our study is timely and is aligned with the goals of the Office of Cancer Survivorship (OCS) and Institute of Medicine (IOM) regarding the management of prostate cancer in the presence of preexisting chronic conditions. To the best of our knowledge, our study is the largest population-based registry study to assess the association between the types of chronic conditions and the cancer stage at diagnosis, initial cancer treatment and clinical outcomes among elderly men with prostate cancer.

As the current study used observational data, the causal associations between the presence of types of chronic condition and cancer stage at diagnosis, and outcomes could not be determined. Our 
study population was restricted to men older than 66 years and older. Therefore, the findings cannot be generalizable to younger men with prostate cancer. In addition, as the presence of chronic conditions were identified using validated codes from the literature, the clinical severity and seriousness of chronic conditions could not be measured which would also be important factors for the initial cancer treatment receipt and outcomes among men with prostate cancer. Furthermore, the current study utilized the registry-linked administrative claims data which have either no information or limited validity of codes to identify body-mass index, exercise and smoking status, which may be associated severity, cancer stage at diagnosis, treatment receipt and clinical outcomes.

\subsection{CONCLUSION}

Elderly men with none of the three types of chronic conditions were more likely to be diagnosed with advanced prostate cancer. Therefore, strategies to reduce the risk of diagnosing prostate cancer at an advanced stage are needed and tailored to such subgroup of population.

All chronic condition categories (except for only mental health conditions and both mental health and respiratory conditions) were associated with an increased likelihood of initial cancer treatment with either RP or RT as compared to all the three types of chronic conditions. Although there is no evidence that RP or RT can be beneficial in long-term for elderly men with prostate cancer and pre-existing chronic conditions, an overwhelming majority of men with chronic conditions received cancer treatment. Future research is needed to identify the long-term benefit and risk of treatment in subgroup of population with different types of chronic conditions.

Although men with some types of chronic conditions were associated with increased risk of sexual dysfunction, no consistent patterns were found for other clinical outcomes by types of chronic conditions. Therefore, men with prostate cancer and some types of chronic conditions i.e. mental health conditions may need heightened surveillance, monitoring and therapies to reduce the risk of sexual dysfunction after the initial cancer treatment. 


\subsection{REFERENCES}

1. Edwards BK, Noone AM, Mariotto AB, Simard EP, Boscoe FP, Henley SJ, et al. Annual Report to the Nation on the status of cancer, 1975-2010, featuring prevalence of comorbidity and impact on survival among persons with lung, colorectal, breast, or prostate cancer. Cancer. 2014 May 1;120(9):1290-314.

2. Xiao H, Tan F, Goovaerts P, Adunlin G, Ali AA, Gwede CK, et al. Impact of Comorbidities on Prostate Cancer Stage at Diagnosis in Florida. Am J Mens Health. 2014 Dec 26.

3. Fleming ST, McDavid K, Pearce K, Pavlov D. Comorbidities and the risk of late-stage prostate cancer. ScientificWorldJournal. 2006 Jul 28;6:2460-70.

4. Oakley-Girvan I, Kolonel LN, Gallagher RP, Wu AH, Felberg A, Whittemore AS. Stage at diagnosis and survival in a multiethnic cohort of prostate cancer patients. Am J Public Health. 2003 Oct;93(10):1753-9.

5. Xu H, Jiang HW, Ding GX, Zhang H, Zhang LM, Mao SH, et al. Diabetes mellitus and prostate cancer risk of different grade or stage: a systematic review and meta-analysis. Diabetes Res Clin Pract. 2013 Mar;99(3):241-9.

6. Prasad SM, Eggener SE, Lipsitz SR, Irwin MR, Ganz PA, Hu JC. Effect of depression on diagnosis, treatment, and mortality of men with clinically localized prostate cancer. J Clin Oncol. 2014 Aug 10;32(23):2471-8.

7. Severi G, Baglietto L, Muller DC, English DR, Jenkins MA, Abramson MJ, et al. Asthma, asthma medications, and prostate cancer risk. Cancer Epidemiol Biomarkers Prev. 2010 Sep;19(9):2318-24.

8. Albertsen PC, Moore DF, Shih W, Lin Y, Li H, Lu-Yao GL. Impact of comorbidity on survival among men with localized prostate cancer. J Clin Oncol. 2011 Apr 1;29(10):1335-41.

9. Daskivich TJ, Fan KH, Koyama T, Albertsen PC, Goodman M, Hamilton AS, et al. Effect of age, tumor risk, and comorbidity on competing risks for survival in a U.S. population-based cohort of men with prostate cancer. Ann Intern Med. 2013 May 21;158(10):709-17.

10. Schymura MJ, Kahn AR, German RR, Hsieh MC, Cress RD, Finch JL, et al. Factors associated with initial treatment and survival for clinically localized prostate cancer: results from the CDCNPCR Patterns of Care Study (PoC1). BMC Cancer. 2010 Apr 19;10:152,2407-10-152.

11. Daskivich TJ, Chamie K, Kwan L, Labo J, Palvolgyi R, Dash A, et al. Overtreatment of men with low-risk prostate cancer and significant comorbidity. Cancer. 2011 May 15;117(10):2058-66.

12. Daskivich TJ, Lai J, Dick AW, Setodji CM, Hanley JM, Litwin MS, et al. Variation in treatment associated with life expectancy in a population-based cohort of men with early-stage prostate cancer. Cancer. 2014 Dec 1;120(23):3642-50.

13. Hamstra DA, Stenmark MH, Ritter T, Litzenberg D, Jackson W, Johnson S, et al. Age and comorbid illness are associated with late rectal toxicity following dose-escalated radiation therapy for prostate cancer. Int J Radiat Oncol Biol Phys. 2013 Apr 1;85(5):1246-53.

14. Sooriakumaran P, Nyberg T, Akre O, Haendler L, Heus I, Olsson M, et al. Comparative effectiveness of radical prostatectomy and radiotherapy in prostate cancer: observational study of mortality outcomes. BMJ. 2014 Feb 26;348:g1502.

15. Nam RK, Cheung P, Herschorn S, Saskin R, Su J, Klotz LH, et al. Incidence of complications other than urinary incontinence or erectile dysfunction after radical prostatectomy or radiotherapy for prostate cancer: a population-based cohort study. Lancet Oncol. 2014 Feb;15(2):223-31.

16. Jayadevappa R, Malkowicz SB, Chhatre S, Johnson JC, Gallo JJ. The burden of depression in prostate cancer. Psychooncology. 2012 Dec;21(12):1338-45.

17. Feinstein A. The pre-therapeutic classification of co-morbidity in chronic disease. J Chron Dis. 1970;23:455-468. .

18. Thomas JA,2nd, Gerber L, Banez LL, Moreira DM, Rittmaster RS, Andriole GL, et al. Prostate cancer risk in men with baseline history of coronary artery disease: results from the REDUCE Study. Cancer Epidemiol Biomarkers Prev. 2012 Apr;21(4):576-81.

19. Platz EA, Till C, Goodman PJ, Parnes HL, Figg WD, Albanes D, et al. Men with Low Serum Cholesterol Have a Lower Risk of High-Grade Prostate Cancer in the Placebo Arm of the Prostate Cancer Prevention Trial. Cancer Epidemiology Biomarkers \& Prevention. 2009 November 01;18(11):2807-13. 
20. Abdollah F, Briganti A, Suardi N, Gallina A, Capitanio U, Salonia A, et al. Does diabetes mellitus increase the risk of high-grade prostate cancer in patients undergoing radical prostatectomy? Prostate Cancer Prostatic Dis. 2011 Mar; 14(1):74-8.

21. Kang J, Chen MH, Zhang Y, Moran BJ, Dosoretz DE, Katin MJ, et al. Type of diabetes mellitus and the odds of Gleason score 8 to 10 prostate cancer. Int J Radiat Oncol Biol Phys. 2012 Mar $1 ; 82(3):$ e463-7.

22. Mitin T, Chen MH, Zhang Y, Moran BJ, Dosoretz DE, Katin MJ, et al. Diabetes mellitus, race and the odds of high grade prostate cancer in men treated with radiation therapy. J Urol. 2011 Dec;186(6):2233-7.

23. Valderas JM, Starfield B, Sibbald B, Salisbury C, Roland M. Defining comorbidity: implications for understanding health and health services. Ann Fam Med. 2009 Jul-Aug;7(4):357-63.

24. Thompson I, Thrasher JB, Aus G, Burnett AL, Canby-Hagino ED, Cookson MS, et al. Guideline for the management of clinically localized prostate cancer: 2007 update. J Urol. 2007

Jun;177(6):2106-31.

25. Mohler J, Bahnson RR, Boston B, Busby JE, D'Amico A, Eastham JA, et al. NCCN clinical practice guidelines in oncology: prostate cancer. J Natl Compr Canc Netw. 2010 Feb;8(2):162-200.

26. Desch CE, Penberthy L, Newschaffer CJ, Hillner BE, Whittemore M, McClish D, et al. Factors that determine the treatment for local and regional prostate cancer. Med Care. 1996 Feb;34(2):152-62. 27. Hall HI, Satariano WA, Thompson T, Ragland KE, Van Den Eeden SK, Selvin S. Initial treatment for prostate carcinoma in relation to comorbidity and symptoms. Cancer. 2002 Dec 1;95(11):2308-15. 28. Berge V, Thompson T, Blackman D. Additional surgical intervention after radical prostatectomy, radiation therapy, androgen-deprivation therapy, or watchful waiting. Eur Urol. 2007 Oct;52(4):103643.

29. Chen AB, D'Amico AV, Neville BA, Earle CC. Patient and treatment factors associated with complications after prostate brachytherapy. J Clin Oncol. 2006 Nov 20;24(33):5298-304.

30. Trantham LC, Nielsen ME, Mobley LR, Wheeler SB, Carpenter WR, Biddle AK. Use of prostatespecific antigen testing as a disease surveillance tool following radical prostatectomy. Cancer. 2013 Oct 1;119(19):3523-30.

31. Wong YN, Mitra N, Hudes G, Localio R, Schwartz JS, Wan F, et al. Survival associated with treatment vs observation of localized prostate cancer in elderly men. JAMA. 2006 Dec 13;296(22):2683-93.

32. Shahinian VB, Kuo YF, Freeman JL, Goodwin JS. Determinants of androgen deprivation therapy use for prostate cancer: role of the urologist. J Natl Cancer Inst. 2006 Jun 21;98(12):839-45.

33. Hu JC, Gold KF, Pashos CL, Mehta SS, Litwin MS. Temporal trends in radical prostatectomy complications from 1991 to 1998. J Urol. 2003 Apr;169(4):1443-8.

34. Begg CB, Riedel ER, Bach PB, Kattan MW, Schrag D, Warren JL, et al. Variations in morbidity after radical prostatectomy. N Engl J Med. 2002 Apr 11;346(15):1138-44.

35. Fortin M, Dubois MF, Hudon C, Soubhi H, Almirall J. Multimorbidity and quality of life: a closer look. Health Qual Life Outcomes. 2007 Aug 6;5:52.

36. Goodman RA, Posner SF, Huang ES, Parekh AK, Koh HK. Defining and measuring chronic conditions: imperatives for research, policy, program, and practice. Prev Chronic Dis. 2013 Apr 25;10:E66.

37. Meraya AM, Raval AD, Sambamoorthi U. Chronic condition combinations and health care expenditures and out-of-pocket spending burden among adults, Medical Expenditure Panel Survey, 2009 and 2011. Prev Chronic Dis. 2015 Jan 29;12:E12.

38. Kadam UT, Uttley J, Jones PW, Iqbal Z. Chronic disease multimorbidity transitions across healthcare interfaces and associated costs: a clinical-linkage database study. BMJ Open. 2013 Jul 19;3(7):10.1136/bmjopen,2013-003109. Print 2013.

39. Vyas A, Pan X, Sambamoorthi U. Chronic Condition Clusters and Polypharmacy among Adults. Int J Family Med. 2012;2012:193168.

40. Yu JB, Soulos PR, Herrin J, Cramer LD, Potosky AL, Roberts KB, et al. Proton versus intensitymodulated radiotherapy for prostate cancer: patterns of care and early toxicity. J Natl Cancer Inst. 2013 Jan 2;105(1):25-32.

41. Piette JD, Kerr EA. The Impact of Comorbid Chronic Conditions on Diabetes Care. Diabetes Care. 2006 March 01;29(3):725-31. 
42. Area Resource File: Overview [Internet].; 2014 []. Available from: http://ahrf.hrsa.gov/overview.htm.

43. Ellis SD, Blackard B, Carpenter WR, Mishel M, Chen RC, Godley PA, et al. Receipt of National Comprehensive Cancer Network guideline-concordant prostate cancer care among African American and Caucasian American men in North Carolina. Cancer. 2013 Jun 15;119(12):2282-90.

44. Terret C, Castel-Kremer E, Albrand G, Droz JP. Effects of comorbidity on screening and early diagnosis of cancer in elderly people. The Lancet Oncology 2015/06;10(1):80-7.

45. Chamie K, Daskivich TJ, Kwan L, Labo J, Dash A, Greenfield S, et al. Comorbidities, treatment and ensuing survival in men with prostate cancer. J Gen Intern Med. 2012 May;27(5):492-9.

46. Nguyen PL, Chen MH, Hoffman KE, Chen RC, Hu JC, Bennett CL, et al. Cardiovascular comorbidity and treatment regret in men with recurrent prostate cancer. BJU Int. 2012 Jul;110(2):2015.

47. Vavassori V, Fiorino C, Rancati T, Magli A, Fellin G, Baccolini M, et al. Predictors for rectal and intestinal acute toxicities during prostate cancer high-dose 3D-CRT: results of a prospective multicenter study. Int J Radiat Oncol Biol Phys. 2007 Apr 1;67(5):1401-10.

48. Tucker SL, Dong L, Bosch WR, Michalski J, Winter K, Mohan R, et al. Late rectal toxicity on RTOG 94-06: analysis using a mixture Lyman model. Int J Radiat Oncol Biol Phys. 2010 Nov 15;78(4):1253-60.

49. Punnen S, Cowan JE, Dunn LB, Shumay DM, Carroll PR, Cooperberg MR. A longitudinal study of anxiety, depression and distress as predictors of sexual and urinary quality of life in men with prostate cancer. BJU Int. $2013 \mathrm{Jul} ; 112(2)$ :E67-75. 
Table 2. 1

Characteristics of Elderly Medicare Beneficiaries with Prostate Cancer Surveillance, Epidemiology and End Results (SEER)-Medicare Linked

Database-2002-2010

\begin{tabular}{lrrl}
\hline \multicolumn{1}{c}{ Characteristics } & Total & \% \\
\hline All & & $\mathbf{1 0 3 , 8 2 0}$ & \\
Types of Chronic Conditions & & \\
CM only & 36,719 & 35.4 \\
MH only & 1,940 & 1.9 \\
RESP only & 5,421 & 5.2 \\
CM + MH & 2,169 & 2.1 \\
CM + RESP & 8,216 & 7.9 \\
MH + RESP & 416 & 0.4 \\
None & 48,009 & 46.2 \\
All Three & 930 & 0.9 \\
\hline
\end{tabular}

Age at diagnosis, years

$\begin{array}{lll}66-74 & 62,020 & 59.7\end{array}$

$\begin{array}{lll}75+ & 41,800 \quad 40.3\end{array}$

Race/ethnicity

$\begin{array}{lll}\text { Whites } & 84,787 & 81.7\end{array}$

$\begin{array}{lrl}\text { African-American } & 11,679 & 11.2\end{array}$

Hispanic/Latino $\quad 2,148 \quad 2.1$

$\begin{array}{lrr}\text { Others } & 5,206 & 5.0\end{array}$

Marital status

Unmarried

$6,954 \quad 6.7$

Married

$69,628 \quad 67.1$

Divorced/Separated

$13,599 \quad 13.1$

Others

$13,639 \quad 13.1$

\begin{tabular}{crr}
\hline \multicolumn{3}{c}{ Enabling Characteristics } \\
\hline Quartile of median census 2000 income & & \\
$\$ 7-\$ 34,522$ & 25,920 & 25.0 \\
$\$ 34,523-46,224$ & 25,959 & 25.0 \\
$\$ 46,229-62,764$ & 25,947 & 25.0 \\
$\$ 62,767-200,008$ & 25,994 & 25.0 \\
Quartile of median census 2000 education & 25,946 & 25.0 \\
$0-8.52$ & 26,057 & 25.1 \\
$8.53-15.16$ & 25,821 & 24.9 \\
$15.17-26.09$ & 25,996 & 25.0 \\
$26.1-100$ & & \\
PSA Screening & 84,681 & 81.6 \\
Yes & 19,139 & 18.4 \\
No & & \\
Visit to a PCP & 76,632 & 73.8 \\
Yes & 27,188 & 26.2 \\
\hline No & & \\
\hline
\end{tabular}


Table 2. 1

Characteristics of Elderly Medicare Beneficiaries with Prostate Cancer Surveillance, Epidemiology and End Results (SEER)-Medicare Linked

Database-2002-2010

\begin{tabular}{|c|c|c|}
\hline Characteristics & Total & $\%$ \\
\hline \multicolumn{3}{|c|}{ External-Environment Characteristics } \\
\hline \multicolumn{3}{|l|}{ SEER-Regions } \\
\hline Northeast & 20,726 & 20.0 \\
\hline South & 25,185 & 24.3 \\
\hline North-Central & 12,907 & 12.4 \\
\hline West & 45,002 & 43.3 \\
\hline \multicolumn{3}{|c|}{ Quartile of Radiation Oncology } \\
\hline 0 to 1 & 27,475 & 26.5 \\
\hline 2 to 6 & 23,281 & 22.4 \\
\hline 7 to 22 & 27,014 & 26.0 \\
\hline 23 to 147 & 26,050 & 25.1 \\
\hline \multicolumn{3}{|c|}{ Quartile of Urology Centers } \\
\hline 0 to 3 & 25,677 & 24.7 \\
\hline 4 to 16 & 25,467 & 24.5 \\
\hline 17 to 44 & 27,053 & 26.1 \\
\hline 45 to 343 & 25,623 & 24.7 \\
\hline \multicolumn{3}{|l|}{ Year of diagnosis } \\
\hline $2002-2005$ & 53,412 & 51.4 \\
\hline 2006-2009 & 50,408 & 48.6 \\
\hline \multicolumn{3}{|c|}{ Cancer Stage at Diagnosis } \\
\hline \multicolumn{3}{|l|}{ Stage } \\
\hline Localized & 98,264 & 94.6 \\
\hline Advanced & 5,556 & 5.4 \\
\hline
\end{tabular}

\begin{tabular}{ccc}
\hline Treatment & 22,864 & 22.0 \\
RP & 48,684 & 46.9 \\
RT & 11,803 & 11.4 \\
Hormone Therapy & 20,469 & 19.7 \\
None & & \\
\hline \multicolumn{2}{c}{ Clinical Outcomes } \\
Bowel Dysfunction & 15,043 & 14.5 \\
Yes & 88,777 & 85.5 \\
No & & \\
Sexual Dysfunction & 16,707 & 16.1 \\
Yes & 87,113 & 83.9 \\
No & & \\
Urinary Dysfunction & 36,368 & 35.0 \\
Yes & 67,452 & 65.0 \\
No & &
\end{tabular}

Notes: Based on the data of 103,820 elderly men aged 66 years and older diagnosed with prostate cancer between 2002 and 2009 using a Surveillance, Epidemiology, and End-Results (SEER)-linked Medicare data.

Abbreviations: CM: Cardio-metabolic conditions; MH: Mental health conditions; PCP: Primary Care Physician; PSA: Prostate-Specific Antigen Test; RESP: Respiratory conditions; RP: Radical Prostatectomy; RT: Radiation Therapy 
Table 2. 2

Characteristics of Elderly Medicare Beneficiaries with Prostate Cancer by Types of Chronic Conditions Surveillance, Epidemiology and End Results (SEER)-Medicare Linked Database-2002-2010

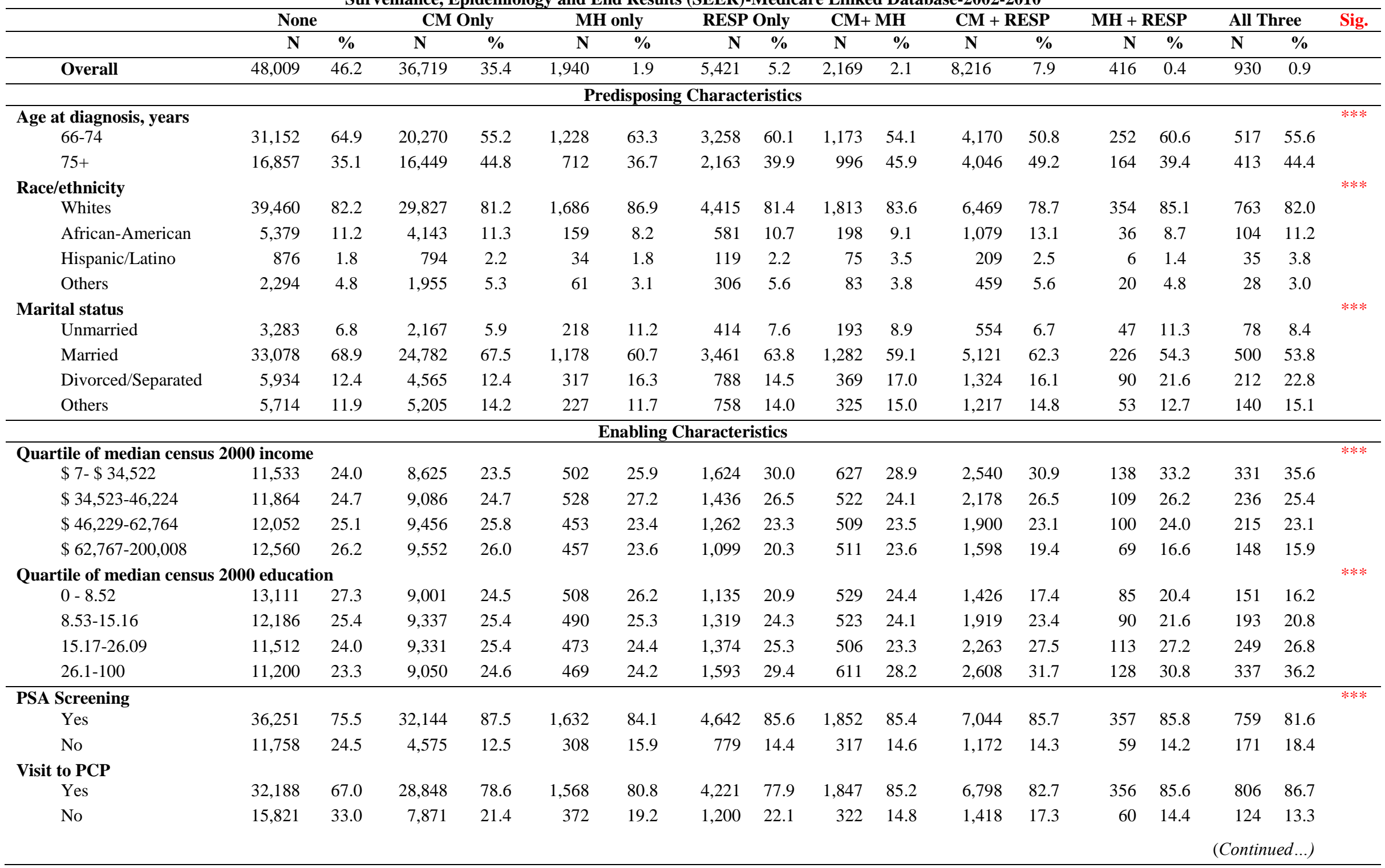


Table 2. 2

Characteristics of Elderly Medicare Beneficiaries with Prostate Cancer by Types of Chronic Conditions

Surveillance, Epidemiology and End Results (SEER)-Medicare Linked Database-2002-2010

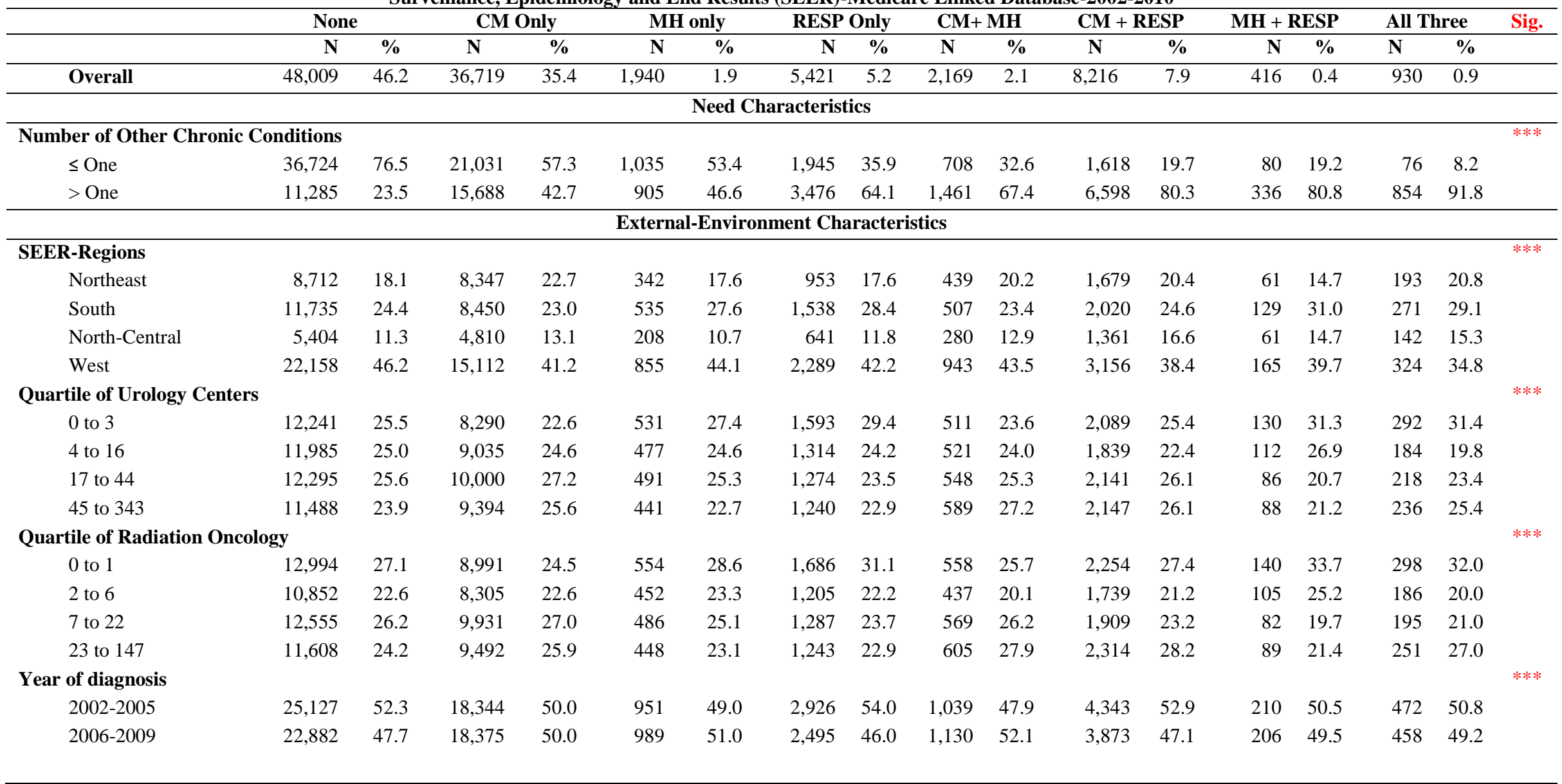

Notes: Based on 103,820 elderly men aged 66 years and older diagnosed with incident prostate cancer between 2002 and 2009. Significant group differences by types of chronic conditions combinations were based on Chi-square tests.

Abbreviations: CM: Cardio-metabolic conditions; MH: Mental health conditions; PCP: Primary Care Physician; PSA: Prostate-Specific Antigen Test; RESP: Respiratory conditions; Sig: Level of Significance; $* * * \mathrm{p}<.001 ; * * .001 \leq \mathrm{p}<.01 ; * .01 \leq \mathrm{p}<.05$ 
Table 2.3

Number and Percent with Advanced Prostate Cancer and Adjusted Odds Ratios (AOR) and 95\% Confidence Intervals from Logistic Regressions for Advanced Stage Diagnosis among Elderly Medicare Beneficiaries with Prostate Cancer Surveillance, Epidemiology and End Results (SEER)-Medicare Linked Database 2002-2010

\begin{tabular}{|c|c|c|c|c|c|c|c|c|}
\hline & \multicolumn{4}{|c|}{ Cancer Stage } & \multicolumn{4}{|c|}{$\begin{array}{c}\text { Logistic Regressions on } \\
\text { Advanced Prostate Cancer Diagnosis }\end{array}$} \\
\hline & \multicolumn{2}{|c|}{ Localized } & \multicolumn{2}{|c|}{ Advanced } & \multirow[b]{2}{*}{ Sig. } & \multirow[b]{2}{*}{ AOR } & \multirow[b]{2}{*}{$95 \% \mathrm{CI}$} & \multirow[b]{2}{*}{ Sig. } \\
\hline & $\mathbf{N}$ & $\%$ & $\mathbf{N}$ & $\%$ & & & & \\
\hline \multicolumn{9}{|c|}{ Types of Chronic Conditions } \\
\hline None & 45,209 & 94.2 & 2,800 & 5.8 & $* * *$ & 1.44 & {$[1.03,2.00]$} & $*$ \\
\hline CM only & 34,916 & 95.1 & 1,803 & 4.9 & & 1.29 & {$[0.93,1.80]$} & \\
\hline MH only & 1,838 & 94.7 & 102 & 5.3 & & 1.33 & {$[0.90,1.95]$} & \\
\hline RSP only & 5,151 & 95.0 & 270 & 5.0 & & 1.32 & {$[0.93,1.88]$} & \\
\hline $\mathrm{CM}+\mathrm{MH}$ & 2,086 & 96.2 & 83 & 3.8 & & 0.89 & {$[0.60,1.33]$} & \\
\hline $\mathrm{CM}+\mathrm{RSP}$ & 7,783 & 94.7 & 433 & 5.3 & & 1.37 & {$[0.97,1.92]$} & \\
\hline $\mathrm{MH}+\mathrm{RSP}$ & 393 & 94.5 & 23 & 5.5 & & 1.24 & {$[0.71,2.17]$} & \\
\hline All Three & 888 & 95.5 & 42 & 4.5 & & Ref & & \\
\hline
\end{tabular}

Notes: Based on 103,820 elderly men aged 66 years and older diagnosed with prostate cancer between 2002 and 2009. Significant differences by types of chronic conditions were based on the log-likelihood test using a logistic regression for adjusted models and chi-square tests for difference in the rates of stages by types of chronic conditions. Adjusted model controlled for predisposing (age, race/ethnicity, marital status), enabling (income, education, access to care, cancer screening), need (number of other chronic conditions), and external-environment factors (county level-radiation unit, urology units, regions, year of diagnosis)

Abbreviations: CM: Cardio-metabolic conditions; MH: Mental health conditions; RESP: Respiratory conditions; Ref: Reference Group; Sig: Level of Significance; *** $\mathrm{p}<.001$; **. $.001 \leq \mathrm{p}<.01 ; * .01 \leq \mathrm{p}<.05$ 
Table 2.4

Number and Percentage with Initial Cancer Treatment by Types of Chronic Conditions Unadjusted and Adjusted Odds Ratios and 95\% Confidence Intervals from Multinomial Logistic Regressions among Elderly Medicare Beneficiaries with Prostate Cancer Surveillance, Epidemiology and End Results (SEER)-Medicare Linked Database 2002-2010

\begin{tabular}{|c|c|c|c|c|c|c|c|c|c|}
\hline & \multicolumn{2}{|c|}{$\mathbf{R P}$} & \multicolumn{2}{|c|}{ RT } & \multicolumn{2}{|c|}{ Hormone Therapy } & \multicolumn{2}{|c|}{ None } & \multirow[t]{2}{*}{ Sig. } \\
\hline & $\mathbf{N}$ & $\%$ & $\mathbf{N}$ & $\%$ & $\mathbf{N}$ & $\%$ & $\mathbf{N}$ & $\%$ & \\
\hline All & 22,864 & 22.0 & 48,684 & 46.9 & 11,803 & 11.4 & 20,469 & 19.7 & \\
\hline \multicolumn{3}{|c|}{ Types of Chronic Conditions } & & & & & & & $* * *$ \\
\hline None & 11,648 & 24.3 & 22,130 & 46.1 & 4,553 & 9.5 & 9,678 & 20.2 & \\
\hline CM Only & 7,300 & 19.9 & 18,008 & 49.0 & 4,487 & 12.2 & 6,924 & 18.9 & \\
\hline MH Only & 473 & 24.4 & 819 & 42.2 & 221 & 11.4 & 427 & 22.0 & \\
\hline RESP Only & 1,212 & 22.4 & 2,537 & 46.8 & 678 & 12.5 & 994 & 18.3 & \\
\hline $\mathbf{C M}+\mathbf{M H}$ & 484 & 22.3 & 895 & 41.3 & 322 & 14.8 & 468 & 21.6 & \\
\hline CM + RESP & 1,459 & 17.8 & 3,758 & 45.7 & 1,334 & 16.2 & 1,665 & 20.3 & \\
\hline MH + RESP & 91 & 21.9 & 182 & 43.8 & 57 & 13.7 & 86 & 20.7 & \\
\hline All Tr & 197 & 21.2 & 355 & 38.2 & 151 & 16.2 & 227 & 24.4 & \\
\hline \multicolumn{10}{|c|}{ Adjusted Model-Initial Cancer Treatment (Ref $=$ No Treatment) } \\
\hline & \multicolumn{3}{|c|}{$\mathbf{R P}$} & \multicolumn{3}{|c|}{ RT } & \multicolumn{3}{|c|}{ Hormone Therapy } \\
\hline & AOR & $95 \% \mathrm{CI}$ & Sig. & AOR & $95 \% \mathrm{CI}$ & Sig. & $\overline{\mathrm{AOR}}$ & $95 \% \mathrm{CI}$ & Sig. \\
\hline \multicolumn{10}{|c|}{ Types of Chronic Conditions } \\
\hline None & 1.32 & {$[1.07,1.61]$} & *** & 1.44 & {$[1.20,1.71]$} & **** & 0.91 & {$[0.73,1.14]$} & \\
\hline CM Only & 1.25 & {$[1.02,1.53]$} & $*$ & 1.69 & {$[1.41,2.01]$} & $* * *$ & 1.12 & {$[0.90,1.40]$} & \\
\hline MH Only & 1.12 & {$[0.88,1.43]$} & & 1.17 & {$[0.95,1.44]$} & & 0.92 & {$[0.70,1.21]$} & \\
\hline RESP Only & 1.36 & {$[1.09,1.69]$} & $* *$ & 1.63 & {$[1.35,1.97]$} & $* * *$ & 1.11 & {$[0.88,1.40]$} & \\
\hline $\mathbf{C M}+\mathbf{M H}$ & 1.20 & {$[0.95,1.53]$} & & 1.27 & {$[1.04,1.57]$} & $*$ & 1.14 & {$[0.88,1.48]$} & \\
\hline CM + RESP & 1.07 & {$[0.87,1.33]$} & & 1.49 & {$[1.24,1.79]$} & $* * *$ & 1.18 & {$[0.94,1.47]$} & \\
\hline MH + RESP & 1.09 & {$[0.75,1.57]$} & & 1.31 & {$[0.96,1.80]$} & & 1.02 & {$[0.68,1.53]$} & \\
\hline All Three & Ref & & & Ref & & & & & \\
\hline
\end{tabular}

Notes: Based on 103,820 elderly men aged 66 years and older diagnosed with prostate cancer between 2002 and 2009. Significant differences in initial cancer treatment by types of chronic conditions were based on chi-square test.

Significant differences by types of chronic conditions were based on the log-likelihood test using a logistic regression for adjusted model. Adjusted model controlled for predisposing (age, race/ethnicity, marital status), enabling (income, education, access to care, cancer screening), need (number of other chronic conditions, cancer stage), and external-environment factors (county level-radiation unit, urology units, regions, year of diagnosis)

Abbreviations: CM: Cardio-metabolic conditions; MH: Mental health conditions; Ref: Reference Group; RESP: Respiratory conditions; Sig: Level of Significance; *** $\mathrm{p}<.001 ; * * .001 \leq \mathrm{p}<.01 ; * .01 \leq \mathrm{p}<.05$ 


\section{Table 2. 5}

Number and Percentage with Poor Clinical Outcomes Unadjusted and Adjusted Odds Ratios and 95\% Confidence Intervals from Logistic Regressions of Clinical Outcomes among Elderly Men with Prostate Cancer by Types of Chronic Conditions Surveillance, Epidemiology and End Results-Medicare Linked Database 20022010

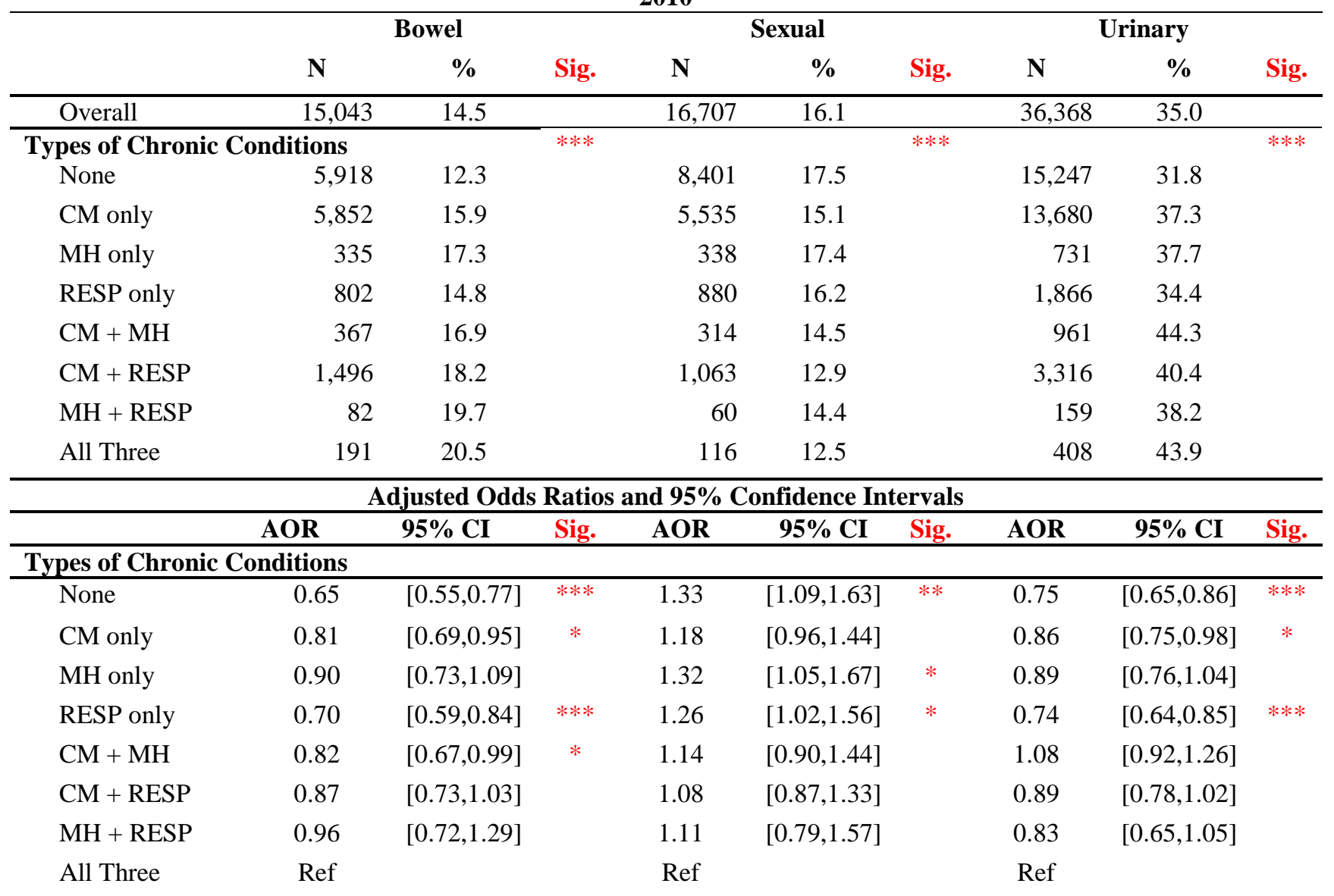

Notes: Based on 103,820 elderly men aged 66 years and older diagnosed with prostate cancer between 2002 and 2009. Significant differences by types of chronic conditions were based on the log-likelihood test using a logistic regression for adjusted models and chi-square tests for difference in the rates of stages by types of chronic conditions. Adjusted model controlled for predisposing (age, race/ethnicity, marital status), enabling (income, education, access to care, cancer screening, cancer treatment), need (number of other chronic conditions, cancer stage), and external-environment factors (county levelradiation unit, urology units, regions, year of diagnosis)

Abbreviations: CM: Cardio-metabolic conditions; MH: Mental health-conditions; RESP: Respiratory conditions; Sig: Level of Significance; $* * * \mathrm{p}<.001 ; * * .001 \leq \mathrm{p}<.01 ; * .01 \leq \mathrm{p}<.05$ 
Appendix 2. 1 Study Cohort Development Flow diagram for Study Population of Elderly Medicare Beneficiaries diagnosed with Prostate Cancer

Men with prostate cancer identified using SEER site recode: 54 and ICD-10-Code: C61.9

$$
\mathrm{N}=358,439
$$

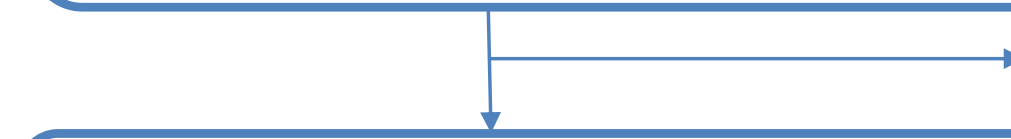

Men diagnosed with prostate cancer as a primary cancer and alive at the time of diagnosis

$$
\mathrm{N}=289,701
$$

Men diagnosed with prostate cancer as primary cancer, malignant tumor and alive at the time of diagnosis

$$
\mathrm{N}=192,501
$$

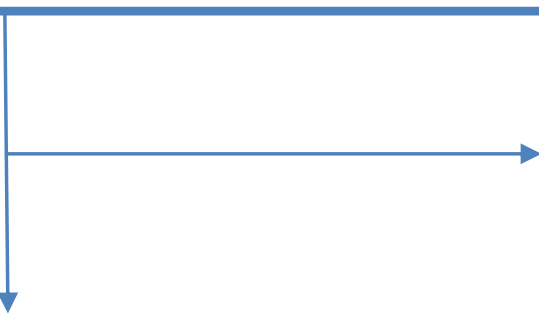

Elderly Men diagnosed with prostate cancer as primary cancer at age 66 years and above, malignant tumor, alive at the time of diagnosis and diagnosed between 2002 to 2009 with 18-month continuous eligibility in Medicare Part A and B and No HMO enrollment during 1 year before diagnosis $\mathrm{N}=109,215$

Elderly Men diagnosed with prostate cancer as primary cancer at age 66 years and above, malignant tumor, alive at the time of diagnosis, diagnosed during 2002 to 2009 , and

$$
\mathrm{N}=\mathbf{1 0 3 , 8 2 0}
$$

- Men with carcinoma in-situ $(\mathrm{N}=40)$

- Men diagnosed with prostate cancer at age 65 years or younger $(\mathrm{N}=97,159)$

Reasons for exclusion:

- Men with cancer having unclear vital status $(\mathrm{N}=41,205)$

- Men with diagnosed prostate cancer before $2002(\mathrm{~N}=1)$

- No Part A and B enrollment and enrollment HMO (N =70,559)

Reasons for exclusion:

- Missing stage $(\mathrm{N}=4,852)$

- Missing information on race $(\mathrm{N}=87)$

- Missing information on education or income $(\mathrm{N}=58)$

- Missing information on county level variables $(\mathrm{N}=393)$ 


\section{Appendix 2.2}

Codes to Identify Radiation Therapy, Surgery, Hormonal and Chemotherapy Utilization Among Elderly Medicare Beneficiaries with Prostate Cancer Using Surveillance, Epidemiology and End Results-Medicare Linked Data 2002-2010

\begin{tabular}{|c|c|c|c|c|c|c|}
\hline \multirow{2}{*}{ Types of Therapy } & \multicolumn{3}{|c|}{ Medicate Claims } & \multicolumn{3}{|c|}{ SEER } \\
\hline & $\begin{array}{l}\text { ICD-9 Procedure } \\
\text { codes in } \\
\text { MedPAR/SAF }\end{array}$ & $\begin{array}{l}\text { ICD-9 diagnostic } \\
\text { codes in } \\
\text { MedPAR/SAF }\end{array}$ & CPT-4 codes in NCH/Carrier file/SAF & $\begin{array}{c}\text { Surgery } \\
\text { codes }\end{array}$ & $\begin{array}{c}\text { Radiation } \\
\text { codes }\end{array}$ & $\begin{array}{c}\text { Revenue } \\
\text { center codes }\end{array}$ \\
\hline \multicolumn{7}{|c|}{ Active Treatment } \\
\hline \multicolumn{7}{|l|}{ Radical Prostatectomy } \\
\hline & $60.2-60.6$ & & $\begin{array}{l}55810,55812,55840,55842,55845, \\
55815,55866\end{array}$ & $30,50,80$ & & \\
\hline \multicolumn{7}{|l|}{ Radiation Therapy } \\
\hline & $\begin{array}{l}\text { 9221-9229, } \\
9227,4604,4610\end{array}$ & V58.0, V66.1, V67.1 & $\begin{array}{l}\text { 55859, 55860, 55862, 55865, 55875, } \\
7687376968,77261-77299,77300,77301 \\
\text { 77305, 77326, 77327, 77328, 77338, } \\
\text { 77401-77499, 77520, 77522, 77523, } \\
\text { 77525, 77750-77799, C1164, C1174, } \\
\text { C1325, C1350 C1700-C1712, C1715- } \\
\text { C1720, C1728 C1790-C1806, C2638 } \\
\text { C2639, C2640 C2641, G0256, G0261, } \\
\text { Q3001, 0073T }\end{array}$ & & $1-5,7$ & 0330 or 0333 \\
\hline \multicolumn{7}{|l|}{ Hormonal therapy } \\
\hline & 62.4 & & $\begin{array}{l}\text { J1950, J9217-J9219, J9202, J9165, 54520, } \\
54521,54522,54530,54535\end{array}$ & 01, & & \\
\hline
\end{tabular}

Note: 3D-CRT, three-dimensional conformal radiation therapy; MIRP- Minimum Invasive Robotic Radical Prostatectomy; CPT-4, Current Procedural Terminology, fourth edition; ICD-9, International Classification of Diseases, ninth revision; IMRT, intensity-modulated radiation therapy; LRP, Laparoscopic Radical Prostatectomy; Open RP, open radical prostatectomy. 


\section{Appendix 2. 3}

Diagnostic and Procedure codes used to Identify Clinical Outcomes-Bowel, Sexual and Urinary Dysfunctions Among Elderly Men with Prostate cancer using SEER-Medicare data from the year 2002 to 2010

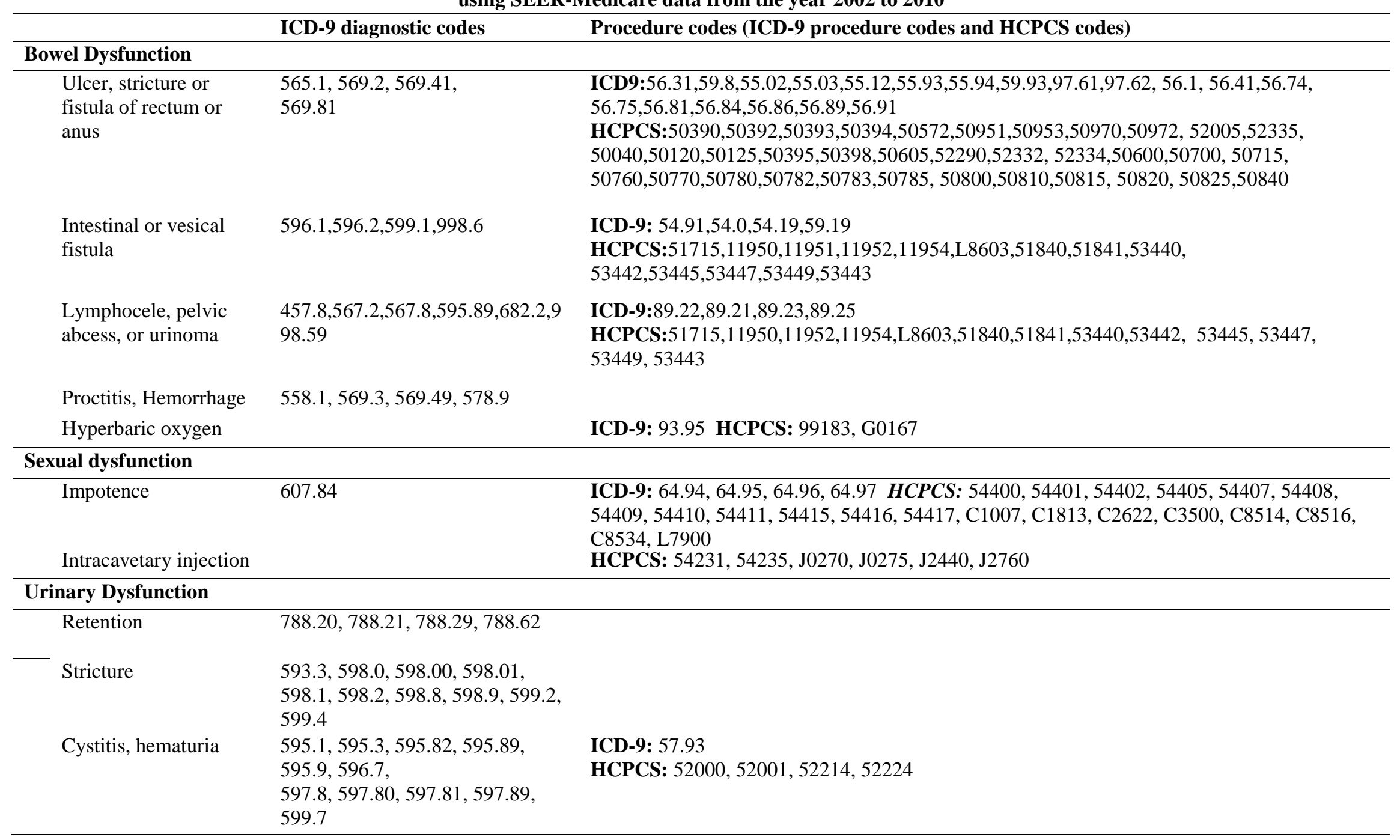


Obstruction

$593.4,596.0,596.3,596.4,596.5$

$596.51,596.52,596.53$

$596.54,596.55,596.59,596.6$

$596.8,596.9,599.6$

Incontinence or

sphincter deficiency

Fistula

$599.82,788.3,788.30,788.31$ $788.32,788.33,788.34,788.35$ $788.36,788.37,788.38,788.39$

$596.1,596.2,599.1,998.6$
ICD-9 : 55.02, 55.03, 55.12, 55.93, 55.94, 56.1, 56.31, 56.41, 56.74, 56.75, 56.81, 56.86, $56.89,56.91,57.0,57.11,57.12,57.17,57.18,57.19,57.21,57.22,57.4,57.41,57.49,57.82$, $57.85,57.89,57.91,57.92,57.93,57.94,57.95,58.0,58.1,58.31,58.39,58.44,58.46,58.47$, $58.5,58.6,58.99,59.8,59.93,59.94,60.21,60.29,60.95,97.61,97.62,97.63,97.64,97.65$, 97.69, 98.19

HCPCS: 50040, 50120, 50125, 50390, 50392-50395, 50398, 50572, 50600, 50605, 50700, 50715, 50760, 50770, 50780, 50782, 50783, 50785, 50800, 50810, 50815, 50820, 50825, 50840, 50951, 50953, 50970, 50972, 51040, 51520, 51600, 51705, 51800, 51820, 51880 52005, 52275, 52276, 52281-52283, 52290, 52310, 52332, 52334, 52335, 52500, 52601, $52612,52614,52620,52620,52630,52640,53000,53010,53020,53400,53405,53410$ $53415,53420,53425,53600,53601,53605,53620,53621,53850,53852$

ICD-9: 58.93, 58.99, 59.3, 59.4, 59.5, 59,6, 59.7, 59.71, 59.72, 59.79

HCPCS: 11950, 11951, 11952, 11954, 51701, 51715, 51840, 51841, 51990, 51992, 53431, 53440, 53442, 53443, 53444, 53445, 53446, 53447,

53448,53449

ICD-9: $56.84,57.83,57.84,58.43$

HCPCS: 45800, 45805, 45820, 45825, 50920, 50930, 53520

HCPCS:E0740,A4335,A5149 
Appendix 2. 4

Codes Used to Identify the Types of Chronic Conditions and List of Other Chronic Conditions Among Elderly Medicare Beneficiaries with Incident Prostate Cancer Surveillance, Epidemiology, End-ResultsMedicare Linked Data of 2002 to 2009

\begin{tabular}{|c|c|}
\hline Types of Chronic Conditions & ICD-9 Diagnostic Codes \\
\hline \multicolumn{2}{|l|}{ Cardio-metabolic Conditions } \\
\hline Coronary Artery Disease & $410,411,412,413,414$ \\
\hline Congestive Heart Failure & $402.01,402.11,402.91,404.01,404.11,404.91,428$ \\
\hline Conductive Disorders & $423,427.31$ \\
\hline Diabetes & 250 \\
\hline \multicolumn{2}{|l|}{ Mental Health Conditions } \\
\hline Depression & $296.2,296.3,311,300.4,311,300.4$ \\
\hline Bipolar Disorders & 296.0, 296.1, 296.4, 296.5, 296.6,296.7, 296.8, 296.9 \\
\hline Schizophrenia & $297,298,299$ \\
\hline Psychoses & 309.81 \\
\hline Anxiety & $300.0,300.2,300.3$ \\
\hline \multicolumn{2}{|l|}{ Respiratory Conditions } \\
\hline COPD and other pulmonary conditions & $490,491,492,495,496,500,501,502,503,504,505,506.4$ \\
\hline Asthma & 493 \\
\hline \multicolumn{2}{|c|}{ List of other Chronic Conditions } \\
\hline \multicolumn{2}{|c|}{$\begin{array}{l}\text { Abuse of drugs, Acute Renal Failure, AIDS, Alcohol abuse, Amputations, Blindness, Cerebrovascular Disease, } \\
\text { Chronic Renal Failure, Cognitive impairment, Dementia, Diverticulitis, End-stage kidney disease, End-stage liver } \\
\text { disease, Epilepsy, Gallstones, Gangrane, Gastritis, Gastroparesis, GERD, Gout, Hemiplegia, Hip Arthoplasty, Low } \\
\text { Back Pain, Multiple sclerosis, Muscular Dystrophy, Nephropathy, Nephrotic syndrome, Osteoarthritis, Parkinson's } \\
\text { Disease, Peptic Ulcers, Peripheral Vascular Disease, Retinopathy, Rheumatoid Arthritis, Spinal Cord Injury } \\
\text { Systemic Lupus Erythematous, Ulcer, Viral Hepatitis }\end{array}$} \\
\hline
\end{tabular}




\section{Chapter 3 \\ Association between Metformin Use and Cancer Stage at Diagnosis and Initial Cancer Treatment among Elderly Medicare Beneficiaries with Diabetes and Prostate Cancer}

\subsection{ABSTRACT}

OBJECTIVE: To examine the associations between metformin use and cancer stage at diagnosis and the initial cancer treatment among elderly men with pre-existing diabetes and incident prostate cancer.

METHODS: A population-based observational cohort study was conducted using the Surveillance, Epidemiology and End-Results (SEER) -Medicare linked database. The study population consisted of elderly men (> 66 years) with pre-existing type 2 Diabetes Mellitus (T2DM) and incident prostate cancer between January 2008 and December $2009(\mathrm{~N}=2,652)$. Cancer stage at diagnosis was categorized into two groups (localized and advanced) based on the American Joint Cancer Committee Tumor-NodeMetastasis (TNM) System classification. The initial cancer treatment during the six months after cancer diagnosis was categorized into four groups using a hierarchy: 1) receipt of radical prostatectomy (RP);2) receipt of radiation therapy (RT); 3) receipt of only hormone therapy; and 4) no cancer treatment.

The key independent variable, metformin use, and other independent variables were measured during the one year period before cancer diagnosis. The independent variables were selected using the Anderson Behavior and Healthcare Utilization Model and consisted of predisposing (age, race/ethnicity, and marital status), enabling (income, education, access to care,), need (severity of diabetes, cancer stage, insulin and other medication use), and external environment factors (region, number of urology and radiation-oncology units measured at the county-level).

Significant unadjusted associations between metformin use and cancer stage at diagnosis and the initial cancer treatment were examined using chi-square tests. The adjusted association between metformin use and cancer stage diagnosis was analyzed using logistic regression after controlling for predisposing, enabling, need, and external environment-related factors. The adjusted association between metformin use and the initial cancer treatment was analyzed using multinomial logistic regression. The reference group for the dependent variable was "no cancer treatment." To control for the observed selection bias in metformin use, inverse probability treatment weights (IPTW) were constructed using logistic regression on 
metformin use. The IPTWs were used in analyses related to the stage of cancer diagnosis and the initial cancer treatment.

RESULTS: In the study cohort, $35.7 \%$ used metformin during the year before diagnosis of prostate cancer. Significant associations were observed between predisposing, enabling, need, and external environment factors and metformin use, suggesting the presence of observed selection bias between metformin users and non-users. Overall, $93.7 \%$ of the study population had a diagnosis of localized prostate cancer and 6.3\% had a diagnosis of advanced prostate cancer. A significantly lower percentage of metformin users were diagnosed with advanced prostate cancer as compared to non-users of metformin $(4.7 \%$ vs. $6.7 \%$, p< 0.03). After controlling for the observed selection bias and other independent variables, metformin use was significantly associated with a reduction in the risk of advanced prostate cancer (Adjusted Odds Ratio, AOR: 0.68, 95\% Confidence Interval, CI: $0.48,0.97)$.

Overall, $72.4 \%$ of the study population received either RP (17.4\%), RT (46.5\%) or hormone therapy (8.6\%) during the six months after the diagnosis of prostate cancer. A higher percentage of metformin users as compared to non-users received RP $(21.3 \%$ vs $16.3 \%, \mathrm{p}<0.001)$. After adjusting for the observed selection bias and other independent variables, metformin users were $45 \%$ more likely to receive $\mathrm{RP}$ as compared to non-users $(\mathrm{AOR}=1.45,95 \% \mathrm{CI}=1.14,1.86)$.

CONCLUSIONS: Among elderly men with T2DM and incident prostate cancer, metformin use was associated with a reduction in the risk of advanced prostate cancer. Metformin users were more likely to receive RP as compared to non-users. No statistically significant relationships were observed between metformin use and RT or hormone therapy.

IMPLICATIONS: The findings from the current study add to the nascent literature on the association between metformin use and the risk for advanced cancer stage and treatment based on observational data. Well-designed, randomized clinical trials are needed to confirm the causal link between metformin use and stage of prostate cancer diagnosis. 


\subsection{INTRODUCTION}

Individuals with diabetes, specifically those with type 2 diabetes mellitus (T2DM) have a higher risk for many cancers such as breast, colon and rectum, endometrium, liver and pancreatic cancers as compared to those without diabetes (1) due to biological mechanisms and shared risk factors (2-4). In preclinical studies, T2DM has been found to be associated with increased levels of plasma insulin, insulin resistance and hyperglycemia, which may have a direct effect on the growth of tumors $(5,6)$ leading to the development of many types of cancers (1).

\subsubsection{Diabetes and Prostate Cancer Risk and Stage at Diagnosis}

In the case of prostate cancer, an inverse relationship between diabetes and cancer risk has been observed (3). However, among men who developed prostate cancer, diabetes was associated with an advanced stage of cancer diagnosis $(7,8)$. Three population-based studies in the Unites States (US) reported that the presence of diabetes was associated with an increased risk for advanced prostate cancer measured either by stage or tumor grade. In case-control studies and cohort studies, men with diabetes were less likely to be diagnosed with localized stage of prostate cancer. The risk ratio (RR) was $0.70,95 \%$ $\mathrm{CI}=0.56-0.86$ for case-control studies and the $\mathrm{RR}$ was $0.72,95 \% \mathrm{CI} 0.67,0.77$ for cohort studies (7). Although the exact biological mechanisms for the link between diabetes and prostate cancer are not known, it is speculated that men with diabetes have low levels of androgen, which may be associated with advanced stage of cancer at diagnosis $(7,8)$.

\subsubsection{Anti-diabetes Drugs (Metformin) and Cancer Stage at Diagnosis}

The main modality of treatment for diabetes is pharmacotherapy with anti-diabetes drugs such as metformin, thiazolidinediones, sulfonylureas, and insulin. All classes of anti-diabetes drugs may indirectly affect the risk of prostate cancer by controlling hyperglycemia. Of special interest is the use of metformin for diabetes management because of its unique actions on insulin resistance and hyperinsulinemia (9) as well as its anti-cancer properties (10-12). A systemic review found that metformin use reduced the risk of prostate cancer among men with diabetes (13) perhaps by regulating adenosine monophosphate-activated protein kinase (AMPK) pathways (10) and mammalian target of rapamycin (mTOR) (12). Based on pre- 
clinical evidence, one can speculate that metformin may also reduce the risk of advanced prostate cancer among men with diabetes and incident prostate cancer (14). This relationship between metformin and advanced prostate cancer diagnosis was explored by one population-based study from Canada (15). The study investigators used a cohort of 119,315 men with diabetes and measured cancer stage using tumor grade. After adjusting for other risk factors, the investigators concluded that metformin use was not associated with the advanced form of cancer diagnosis (23). The investigators of the study did not control for severity of diabetes, which may have affected the findings. Controlling for diabetes severity is important because individuals with severe diabetes have micro and macro-vascular complications and may have adverse pathological profiles (16). Therefore, diabetes severity may alter the relationship between metformin use and cancer stage at diagnosis. Furthermore, the study did not control for the observed selection bias between metformin users and non-users; such bias may lead to misleading findings on the association between metformin use and cancer stage at diagnosis. Thus, there is a need for populationbased studies to examine the relationship between metformin use and cancer stage at diagnosis that control for a comprehensive set of risk factors and the observed selection bias between metformin users and nonusers.

\subsubsection{Metformin Use and the Initial cancer treatment}

Metformin use may also affect the types of initial cancer treatment. The initial cancer treatment is often determined by the stage of diagnosis, life expectancy of an individual and pre-existing chronic conditions (17). However, in the light of recent findings about the anti-cancer properties of metformin, physicians may take into account the use of metformin when deciding the types of initial cancer treatment among men with diabetes and prostate cancer. For example, metformin down regulates the androgenreceptors levels, which in turn maximizes the anti-cancer properties of hormone therapy (18). Radiation therapy along with metformin has been found to inhibit the progression of cancer and reduce mortality among men with prostate cancer (19). Therefore, among men with prostate cancer and diabetes, metformin users may be more likely to receive radiation therapy or hormone therapy as compared to non-users. However, there are no population-based studies examining the association between metformin use and the types of initial cancer treatment among men with diabetes and incident prostate cancer. 
Therefore, the primary objective of the study is to investigate the association between metformin use and cancer stage at diagnosis and the initial cancer treatment among elderly fee-for-service Medicare beneficiaries with T2DM and incident prostate cancer.

\subsection{CONCEPTUAL FRAMEWORK}

We utilized the ABM model (20) described in chapter 1, for selection of independent variables. The depiction of the current study-specific independent characteristics are provided in Figure 3.1.

Figure 3.1: Study Theoretical Framework: Adaptation of Andersen Behavioral Model for Aim 2

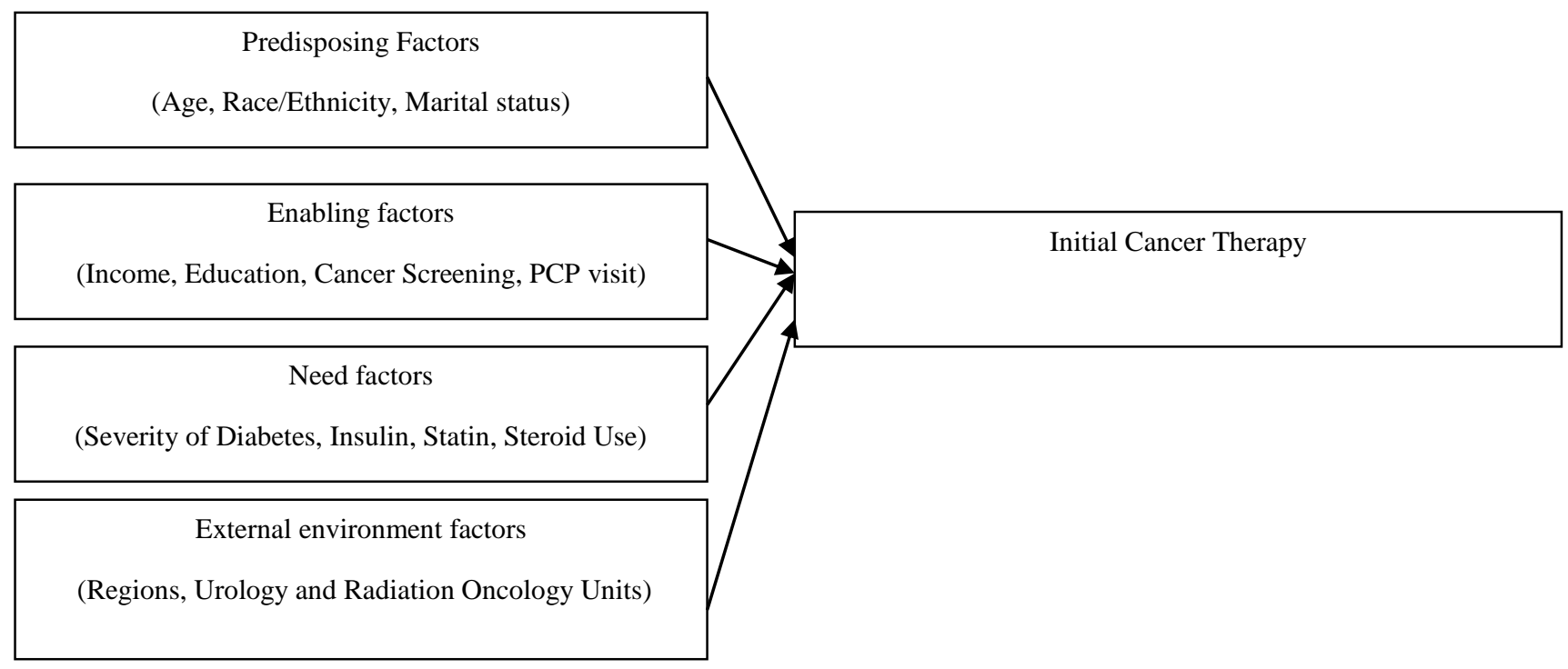

\subsection{METHODS}

\subsubsection{Study Design}

A cohort study design was adopted with a baseline and a follow-up period as depicted in Figure 3.2. The index date was defined as the date of diagnosis of prostate cancer. The baseline period consisted of 12 months before the index date and the follow-up period consisted of 6 months after the index date. Diabetes, metformin use and other independent variables were identified during the baseline period. The types of initial cancer treatment were identified during the follow-up period. 
Figure 3. 2 Schematic Presentation of Study Design for Aim 2

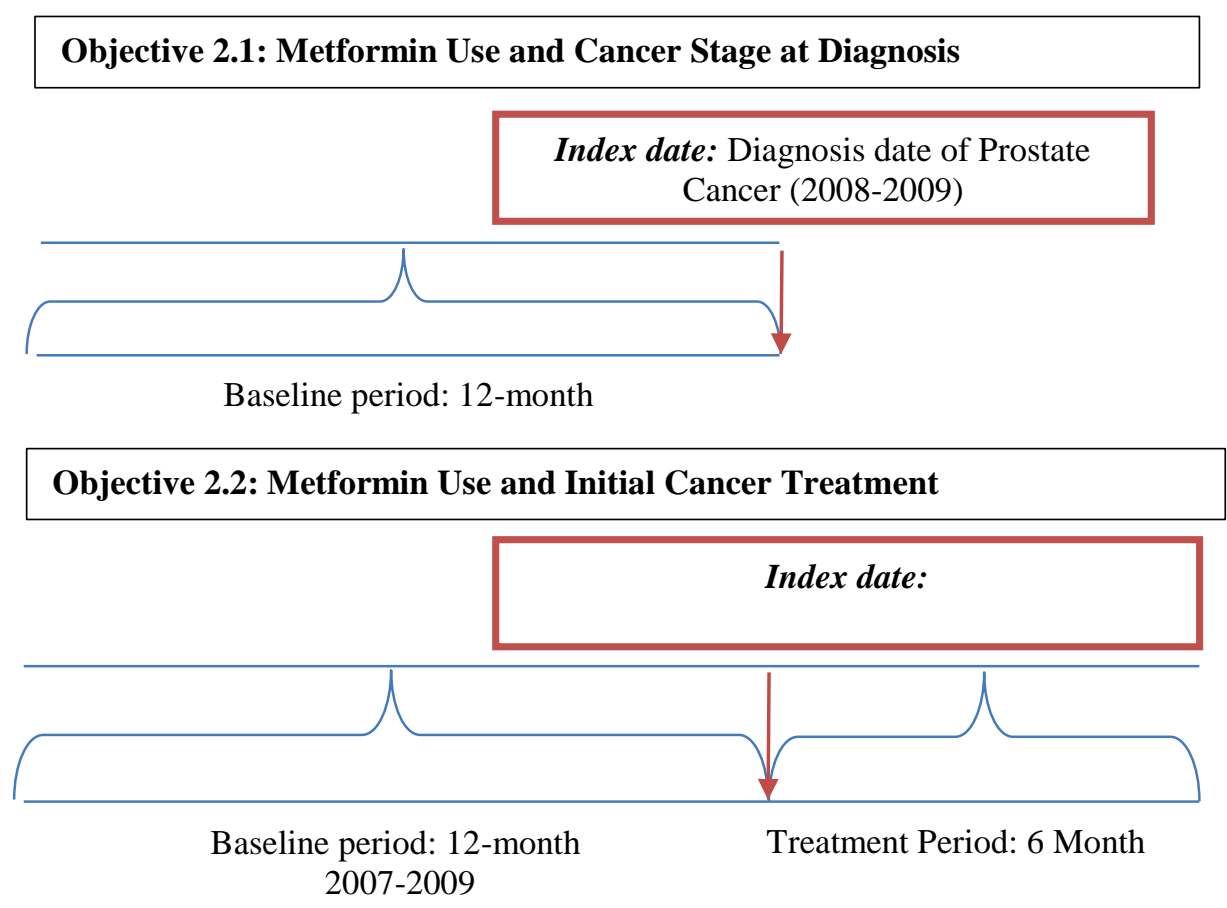

\subsubsection{Data Source}

Data were derived from the SEER-Medicare linked database, details of which are provided in the Chapter 1.

\subsubsection{Study Cohort}

The study cohort comprised of 74,791 men with incident prostate cancer diagnosed between 2008 and 2009. We excluded 7,763 cases for the following reasons: diagnosis of prostate cancer during the autopsy; the presence of multiple cancers; carcinoma in-situ; aged 65 years and younger; died during the study period; enrolled in Medicare Health Maintenance Organizations; not continuously enrolled in Medicare PART A, B, and D during the study period, and missing cancer stage at diagnosis. The details on the study cohort selection process are provided in Appendix 3.1.

After all the exclusions, the final study cohort consisted of 2,652 elderly men with Type 2 diabetes Mellitus (T2DM) and incident prostate cancer. The presence of T2DM was identified using at least one inpatient visit or two or more physician visits with a primary or a secondary diagnosis codes for T2DM (ICD-9-CM codes: $250 . x 0$ or 250. $x 2$ ) during the baseline period. 


\subsubsection{Cancer Stage at Diagnosis}

The American Joint Committee on Cancer (AJCC) Tumor-Node-Metastases (TNM) classification was used to identify the stage of prostate cancer from the PEDSF file. Based on the AJCC-TNM systems, men were classified as having localized cancer stage if they had $\mathrm{T} 1$ or $\mathrm{T} 2$ clinical stage with no regional lymph node (NX-N0) involvement and absence of any distant metastasis (M0) (21). Men were classified as having advanced cancer if they were diagnosed with $\mathrm{T} 3$ or T4 clinical stage with or without regional lymph node (N1) or distant metastasis (M1).

\subsubsection{The initial cancer treatment}

The initial cancer treatment categories were identified using both SEER-PEDSF file and inpatient, outpatient and carrier files using validated algorithms $(22,23,24)$ during the follow-up period. The details on diagnosis and procedure codes used to identify types of cancer treatment are provided in Appendix 2.2. The initial cancer treatment receipt was classified using a hierarchy. Men who received radical prostatectomy (RP) were classified into one group; among men who did not receive $\mathrm{RP}$, receipt of radiation therapy (RT) was considered; among men who did not receive RT, hormone therapy was considered. The final classification consisted of men who: 1) received RP; 2) received RT alone or RT with hormone therapy; 3) those who received only hormone therapy, and 4) those who did not receive any RP, RT or hormone therapy. This hierarchical classification was adopted to reflect clinical practices in the real-world settings $(25,26)$.

\subsubsection{Key Independent Variable}

\subsubsection{Metformin Use}

Metformin use was identified using the Medicare Part-D files. Metformin prescriptions were identified using the national drug codes (NDCs). Men with at least one prescription for metformin during the baseline period were considered as metformin users and men without any prescriptions for metformin were considered as non-users. 


\subsubsection{Other Independent Variables}

\subsubsection{Predisposing characteristics}

Predisposing characteristics consisted of age at diagnosis, race, and marital status. These were identified from the PEDSF file. Age at the time of diagnosis was categorized into two groups (66 to 74 years, $\geq 75$ years). Race/ethnicity was categorized into four groups: White, African American, Latino, and other. Marital status was categorized into four groups: Married, Divorced/Separated, Unmarried, and Others.

\subsubsection{Enabling characteristics}

Enabling characteristics were: education, income, PSA test, visits to primary care physicians, and medication coverage gap. Median income and median education at the census tract of residence were derived from the PEDSF file. Income and education were measured by quartiles. The receipt of PSA test was identified using the following HCPCS codes: 84152, 84153, 84154, and G0103 using the Medicare carrier files during the baseline period. The presence or absence of primary care visits during the baseline was identified using the provider specialty codes (27).

Medication coverage gap (i.e. donut hole) was identified at the time of prostate cancer diagnosis. Coverage gap was defined as follows: 1) donut hole not reached at the time of cancer diagnosis; 2) in donut hole at the time of cancer diagnosis; and 3) out of donut hole at the time of cancer diagnosis.

\subsubsection{Need characteristics}

The severity of diabetes, insulin use, statins use and corticosteroid use were considered as need factors. The Diabetes Complications Severity Index (DCSI) was calculated using the modified algorithm by Chang et al. (28). The DSCI is based on seven categories: retinopathy, nephropathy, neuropathy, cerebrovascular, cardio-vascular, peripheral vascular disease, and metabolic conditions. Based on the severity of particular types of complications, a score of 1 or 2 were assigned to each of the seven categories, with a total DCSI score ranging from 0 to 13. The DCSI scores were grouped into quartiles. Details of the ICD-9-CM codes and the scoring algorithm are provided in Appendix 3.2. The use of 
insulin, statins, and corticosteroids were identified using the NDCs recorded in the Medicare Part D files during the baseline period.

\subsubsection{External environment characteristics}

The SEER data has 18 registries/regions which were categorized into four regions:1) Northeast with two registries of Connecticut, and New Jersey; 2) South, with five registries of Kentucky, Louisiana, Atlanta, Rural Georgia, Greater Georgia; 3) North-Central with two registries of Detroit and Iowa; and 4) West with Hawaii, New Mexico, Seattle-Puget Sound, Utah, San Francisco-Oakland, San Jose-Monterey, Los Angeles, Greater California, Arizona, Alaska, and Cherokee Nation (29). The number of radiation oncology units, and urology units at the county-level (converted into quartiles) were also included (29).

\subsection{STATISTICAL ANALYSES}

Significant group differences in the study population characteristics by metformin use was examined with chi-square tests. A binary logistic regression was used to determine the associations between predisposing, enabling, need, and external environment characteristics and metformin use. Cstatistics and area under the curve were used to assess the model fit. Logistic regression was used to derive inverse probability treatment weights (IPTW) and these IPTWs were used to control for the observed selection bias in regressions on the cancer stage and the initial cancer treatment.

Significant unadjusted associations between metformin use and cancer stage at diagnosis and the initial cancer treatment were examined with chi-square tests. The IPTW-adjusted multivariable logistic regression and multinomial logistic regression were used to analyze the relationship between metformin use and cancer stage at diagnosis and the initial cancer treatment respectively. As the odds ratios and relative risk are approximately similar for the events with low prevalence such as advanced prostate cancer $(\leq 10 \%)(30)$, these terms risk ratio or odds ratio of advanced prostate cancer were used interchangeably. All statistical analyses were carried out using Statistical Analysis System (SAS) version 9.4 (SAS Institute Inc., Cary, NC). 


\subsection{RESULTS}

\subsubsection{Description of the Study Cohort}

The study cohort consisted of 2,757 elderly men with pre-existing diabetes and incident prostate cancer between 2008 and 2009. Table 3.1 represents the characteristics of the study cohort. An overwhelming majority of men were whites (92.3); 59.7\% were diagnosed with prostate cancer between the ages of 66 and 74 years; $58.6 \%$ were married and $46.2 \%$ resided in the Western region of the US. Nearly three-quarters $(70.0 \%)$ of study cohort had a primary care visit. An overwhelming majority (91.1\%) had PSA tests during the baseline period.

\subsubsection{Description of the Study Cohort by Metformin Use}

Table 3.1 also summarizes the characteristics of the study cohort by metformin use. Overall, $35.6 \%$ of the study cohort had at least one prescription of metformin during the baseline period. Significant differences in predisposing, enabling, need and external environment factors by metformin use were observed.

Table 3.2 describes the adjusted odds ratios (AOR) and 95\% CI for the metformin use among elderly men with diabetes and prostate cancer. Elderly men aged 66 to 74 years as compared to those with 75 years and older (AOR: 1.31, 95\% CI: 1.10, 1.56), Latinos as compared to Whites (AOR: $1.62,95 \%$ CI: $1.12,2.34$ ), those who received insulin as compared to no insulin (AOR: $2.95,95 \%$ CI: $1.94,4.49$ ), those who received statins as compared to no statins (AOR: 1.94, 95\% CI: 1.63, 2.31) were more likely to receive metformin. Whereas, elderly men with a severe DCSI score (4 to 13) were less likely to receive metformin as compared to those with zero or one DCSCI score (AOR: 0.65, 95\% CI: 0.51, 0.84).

\section{$\underline{\text { 3.6.3 Metformin and Cancer Stage at Diagnosis }}$}

Table 3.3 describes the relationship between metformin use and cancer stage at diagnosis among elderly men with prostate cancer and diabetes. Overall, $93.7 \%$ of the study population were diagnosed with localized prostate cancer; $6.3 \%$ were diagnosed with advanced prostate cancer. We did not observe a statistically significant difference in the rates of localized or advanced prostate cancer at diagnosis by metformin use. 
Table 3.3 also reports unadjusted odds ratios (OR) and adjusted ORs (AOR) from IPTW logistic regressions for the advanced prostate cancer among elderly men with diabetes and prostate cancer. In unadjusted logistic regression, we observed a significant association between metformin and risk of advanced prostate cancer at diagnosis (OR: $0.69,95 \%$ CI: 0.49, 0.95). After adjusting for predisposing, enabling, need and external-environment factors among elderly men with prostate cancer and diabetes, metformin use was significantly associated with a reduction in the risk of advanced prostate cancer (AOR: $0.68,95 \%$ CI: $0.48,0.97)$.

\subsubsection{Metformin and the Initial Cancer Treatment}

Overall, $72.4 \%$ of the study population received either RP/RT or hormone therapy during the six months after the cancer diagnosis. RT was the most received initial cancer treatment in the study population $(46.5 \%)$, followed by RP $(17.4 \%)$ and only hormone therapy $(8.6 \%)$. Nearly one quarter of the study population did not receive any cancer treatment. A greater proportion of the elderly men using metformin received RP (21.3\%) as compared to those who did not use metformin (16.6\%), whereas, a lower proportion of elderly men using metformin (23.8\%) did not receive any cancer treatment as compared to those who did not use metformin (28.5\%).

Table 3.4 displays the ORs and AORs from multinomial logistic regressions on the receipt of active treatment of either RP, RT or only hormone therapy among elderly men with prostate cancer and diabetes. In unadjusted analysis, metformin users were $54 \%$ more likely to receive the RP as the initial cancer therapy (OR: $1.54,95 \%$ CI: $1.24,1.92)$, and RT (OR: $1.20,95 \%$ CI: $1.04,1.44)$ as compared to non-metformin users among elderly men with prostate cancer. Even after controlling for predisposing, enabling, need and external environment-related factors, metformin users were $45 \%$ more likely to receive RP as compared non-metformin users among elderly men with diabetes and prostate cancer (AOR: 1.45, 95\% CI: $1.14,1.86)$. The use of metformin was not associated with RT or only hormone therapy among elderly men with diabetes and prostate cancer. 


\subsection{DISCUSSION}

The current study is the first largest population-based study to examine whether the risk of advanced prostate cancer diagnosis is reduced with metformin use among elderly men with pre-existing T2DM and incident prostate cancer in the US. After controlling for the observed section bias between metformin users and non-users and other independent variables, the risk for advanced stage cancer diagnosis is reduced with metformin use among elderly men with T2DM and incident prostate cancer. The current study addressed the limitations of the single population-based examination of metformin use and the cancer stage by incorporating a validated diabetes severity complications index and controlling for the observed selection bias. The current study findings are consistent with the pre-clinical evidence on the role of metformin in prevention of advanced prostate cancer (22) (10). If the findings of the current study are confirmed by other population-based studies, randomized clinical trials can be conducted to establish the causal link between metformin use and risk of advanced prostate cancer diagnosis.

With regards to the initial cancer treatment, the current study findings revealed that the initial cancer treatment with RP or RT or hormone therapy was very common (72.4\%) among elderly men with T2DM and incident prostate cancer. In the absence of clear guidelines for management of prostate cancer among men with diabetes, the choice of initial cancer treatment has been a subject of debate. Active treatment is often not preferred because elderly men with T2DM and incident prostate cancer may be at higher risk for mortality due to cardiovascular conditions than morality due to prostate cancer (31). Additionally, RP can lead to increased surgical complications as well as greater risk of bowel, and urinary dysfunction $(32,33)$. Therefore, the benefits of RP should be considered after taking into account the harms associated with the active treatment for prostate cancer.

Although the clinical evidence suggests that RT (19) or hormone therapy (34) augmented with metformin are associated with better clinical outcomes as compared to those without metformin, the current study did not find a statistically significant associations between metformin use and the receipt of RT or hormone therapy. It is plausible that the choice of initial cancer treatment may be solely determined based on age, stage of cancer, and pre-existing chronic conditions and metformin use may not have been considered. The reasons for the positive association between metformin use and RP could not be examined 
due to the inherent limitations of the claims data analysis. In the future, studies need to be conducted to identify the reasons for the higher rates of RP among metformin users as compared to non-users, among elderly men with T2DM and incident prostate cancer.

It should be noted that the current study controlled for the observed selection bias because metformin users and non-users were significantly different with respect to their predisposing, enabling, need, and external environment characteristics. Without adjustments for the observed selection bias, there was not a statistically significant difference in the cancer stage between metformin users and non-users. Therefore, accounting for the observed selection bias is important in establishing an association between metformin use and the reduction in the risk of advanced prostate cancer diagnosis.

The current study has a number of strengths. First, the large cohort size and high quality data on the clinical and pathological features of cancer at the time of diagnosis enabled us to examine not only the association between metformin use and the cancer stage diagnosis but also the initial choice of cancer treatment (35). Furthermore, the inclusion of variable on severity of diabetes using a validated method enabled to control and relate the effect of severity of disease on the risk of advanced prostate cancer at diagnosis and receipt of initial cancer treatment.

The current study has some limitations as well. The prescription claims for metformin and other drugs were used. Filling the prescriptions cannot be equated to the actual use of these drugs. The study population consisted of elderly men residing in SEER-Regions and enrolled as fee-for-services Medicare Beneficiaries, therefore, one cannot generalize the study finding to younger men or all Medicare beneficiaries with incident prostate cancer in the US. Information on many important prognostic factors such as body-mass index and smoking could not be adjusted; these factors may be associated with an increased risk of advanced prostate cancer diagnosis. Duration of metformin use could not be adjusted due to data limitations. Future studies need to examine whether a greater duration of metformin use is associated with a decrease in the risk of advanced prostate cancer at diagnosis. As the study population was restricted to elderly men with T2DM, the current study findings cannot be generalized to men without T2DM and incident prostate cancer. 


\subsection{CONCLUSIONS}

Metformin use was associated with a statistically significant reduction in the risk advanced prostate cancer diagnosis among elderly men with T2DM and incident prostate cancer. Among elderly men with T2DM and incident prostate cancer, RP was more likely among metformin users as compared to non-users. No statistically significant associations were observed with regard to metformin use and RT or hormone therapy.

The current study findings highlight the need for additional studies in this area. Other populationbased studies need to be conducted to confirm the study findings. If confirmed, randomized controlled trials can be carried out to examine the causal link between metformin use and the risk of advanced prostate cancer diagnosis. If the findings of the current study are replicated, metformin may be used as a preventive therapy to reduce the risk of aggressive prostate cancer among elderly men with T2DM and incident prostate cancer. Future studies should be conducted to evaluate clinical outcomes of metformin use among elderly men with prostate cancer and diabetes. 


\subsection{REFERENCES}

1. Giovannucci E, Harlan DM, Archer MC, Bergenstal RM, Gapstur SM, Habel LA, et al. Diabetes and cancer: a consensus report. Diabetes Care. 2010 Jul;33(7):1674-85.

2. Abdollah F, Briganti A, Suardi N, Gallina A, Capitanio U, Salonia A, et al. Does diabetes mellitus increase the risk of high-grade prostate cancer in patients undergoing radical prostatectomy? Prostate Cancer Prostatic Dis. 2011 Mar;14(1):74-8.

3. Bansal D, Bhansali A, Kapil G, Undela K, Tiwari P. Type 2 diabetes and risk of prostate cancer: a meta-analysis of observational studies. Prostate Cancer Prostatic Dis. 2013 Jun;16(2):151,8, S1.

4. Kang J, Chen MH, Zhang Y, Moran BJ, Dosoretz DE, Katin MJ, et al. Type of diabetes mellitus and the odds of Gleason score 8 to 10 prostate cancer. Int J Radiat Oncol Biol Phys. 2012 Mar 1;82(3):e463-7.

5. Ribeiro AM, Pereira S, Andrade S, Costa M, Lopes C, Aguas AP, et al. Insulin prevents leptin inhibition of RM1 prostate cancer cell growth. Pathol Oncol Res. 2012 Apr;18(2):499-507.

6. Lubik AA, Gunter JH, Hendy SC, Locke JA, Adomat HH, Thompson V, et al. Insulin increases de novo steroidogenesis in prostate cancer cells. Cancer Res. 2011 Sep 1;71(17):5754-64.

7. Xu H, Jiang HW, Ding GX, Zhang H, Zhang LM, Mao SH, et al. Diabetes mellitus and prostate cancer risk of different grade or stage: a systematic review and meta-analysis. Diabetes Res Clin Pract. 2013 Mar;99(3):241-9.

8. Khera M, Crawford D, Morales A, Salonia A, Morgentaler A. A new era of testosterone and prostate cancer: from physiology to clinical implications. Eur Urol. 2014 Jan;65(1):115-23.

9. Ashokkumar N, Pari L, Rao C. Effect of N-benzoyl-D-phenylalanine and metformin on insulin receptors in neonatal streptozotocin-induced diabetic rats: studies on insulin binding to erythrocytes. Arch Physiol Biochem. 2006 Jul;112(3):174-81.

10. Hadad SM, Fleming S, Thompson AM. Targeting AMPK: a new therapeutic opportunity in breast cancer. Crit Rev Oncol. 2008;67(1):1-7.

11. Huang X, Wullschleger S, Shpiro N, McGuire V, Sakamoto K, Woods Y, et al. Important role of the LKB1AMPK pathway in suppressing tumorigenesis in PTEN-deficient mice. Biochem J. 2008 Portland Press Limited;412(2):211-21.

12. Bitting RL, Armstrong AJ. Targeting the PI3K/Akt/mTOR pathway in castration-resistant prostate cancer. Endocr Relat Cancer. 2013 May 20;20(3):R83-99.

13. Deng D, Yang Y, Tang X, Skrip L, Qiu J, Wang Y, et al. Association between metformin therapy and incidence, recurrence and mortality of prostate cancer: evidence from a meta-analysis. Diabetes Metab Res. 2015:n/a,n/a. 14. Ben Sahra I, Laurent K, Loubat A, Giorgetti-Peraldi S, Colosetti P, Auberger P, et al. The antidiabetic drug metformin exerts an antitumoral effect in vitro and in vivo through a decrease of cyclin D1 level. Oncogene. 2008 Jun 5;27(25):3576-86.

15. Margel D, Urbach D, Lipscombe LL, Bell CM, Kulkarni G, Austin PC, et al. Association between metformin use and risk of prostate cancer and its grade. J Natl Cancer Inst. 2013 Aug 7;105(15):1123-31.

16. Müller H, Raum E, Rothenbacher D, Stegmaier C, Brenner H. Association of Diabetes and Body Mass Index with Levels of Prostate-Specific Antigen: Implications for Correction of Prostate-Specific Antigen Cutoff Values? Cancer Epidemiology Biomarkers \& Prevention. 2009 May 01;18(5):1350-6.

17. Mohler JL, Kantoff PW, Armstrong AJ, Bahnson RR, Cohen M, D'Amico AV, et al. Prostate cancer, version 2.2014. J Natl Compr Canc Netw. 2014 May;12(5):686-718.

18. Shen M, Zhang Z, Ratnam M, Dou QP. The interplay of AMP-activated protein kinase and androgen receptor in prostate cancer cells. J Cell Physiol. 2014 Jun;229(6):688-95.

19. Spratt DE, Zhang C, Zumsteg ZS, Pei X, Zhang Z, Zelefsky MJ. Metformin and prostate cancer: reduced development of castration-resistant disease and prostate cancer mortality. Eur Urol. 2013 Apr;63(4):709-16.

20. Andersen RM. Revisiting the behavioral model and access to medical care: does it matter? J Health Soc Behav. 1995 Mar;36(1):1-10.

21. Thompson I, Thrasher JB, Aus G, Burnett AL, Canby-Hagino ED, Cookson MS, et al. Guideline for the management of clinically localized prostate cancer: 2007 update. J Urol. 2007 Jun;177(6):2106-31.

22. Trantham LC, Nielsen ME, Mobley LR, Wheeler SB, Carpenter WR, Biddle AK. Use of prostate-specific antigen testing as a disease surveillance tool following radical prostatectomy. Cancer. 2013 Oct 1;119(19):3523-30.

23. Wong YN, Mitra N, Hudes G, Localio R, Schwartz JS, Wan F, et al. Survival associated with treatment vs observation of localized prostate cancer in elderly men. JAMA. 2006 Dec 13;296(22):2683-93.

24. Shahinian VB, Kuo YF, Freeman JL, Goodwin JS. Determinants of androgen deprivation therapy use for prostate cancer: role of the urologist. J Natl Cancer Inst. 2006 Jun 21;98(12):839-45.

25. Godley PA, Schenck AP, Amamoo MA, Schoenbach VJ, Peacock S, Manning M, et al. Racial differences in mortality among Medicare recipients after treatment for localized prostate cancer. J Natl Cancer Inst. 2003 Nov 19;95(22):1702-10.

26. Cohen JH, Schoenbach VJ, Kaufman JS, Talcott JA, Schenck AP, Peacock S, et al. Racial differences in clinical progression among Medicare recipients after treatment for localized prostate cancer (United States). Cancer Causes Control. 2006 Aug;17(6):803-11. 
27. Yu JB, Soulos PR, Herrin J, Cramer LD, Potosky AL, Roberts KB, et al. Proton versus intensity-modulated radiotherapy for prostate cancer: patterns of care and early toxicity. J Natl Cancer Inst. 2013 Jan 2;105(1):25-32. 28. Chang HY, Weiner JP, Richards TM, Bleich SN, Segal JB. Validating the adapted Diabetes Complications Severity Index in claims data. Am J Manag Care. 2012 Nov;18(11):721-6.

29. Area Resource File: Overview [Internet].; 2014 []. Available from: http://ahrf.hrsa.gov/overview.htm. 30. Zhang J, Yu KF. What's the relative risk? A method of correcting the odds ratio in cohort studies of common outcomes. JAMA. 1998 Nov 18;280(19):1690-1.

31. D'Amico AV, Braccioforte MH, Moran BJ, Chen M. Causes of Death in Men With Prevalent Diabetes and Newly Diagnosed High- Versus Favorable-Risk Prostate Cancer. International Journal of Radiation Oncology â€६ Biology â€ $\notin$ Physics 2015/06;77(5):1329-37.

32. Resnick MJ, Barocas DA, Morgans AK, Phillips SE, Chen VW, Cooperberg MR, et al. Contemporary prevalence of pretreatment urinary, sexual, hormonal, and bowel dysfunction: Defining the population at risk for harms of prostate cancer treatment. Cancer. 2014;120(8):1263-71.

33. Potosky AL, Davis WW, Hoffman RM, Stanford JL, Stephenson RA, Penson DF, et al. Five-Year Outcomes After Prostatectomy or Radiotherapy for Prostate Cancer: The Prostate Cancer Outcomes Study. Journal of the National Cancer Institute. 2004 September 15;96(18):1358-67.

34. Shen M, Zhang Z, Ratnam M, Dou QP. The interplay of AMP-activated protein kinase and androgen receptor in prostate cancer cells. J Cell Physiol. 2014 Jun;229(6):688-95.

35. Warren JL, Klabunde CN, Schrag D, Bach PB, Riley GF. Overview of the SEER-Medicare data: content, research applications, and generalizability to the United States elderly population. Med Care. 2002 Aug;40(8 Suppl):IV,3-18. 
Table 3. 1

Characteristics of the Study Cohort Elderly Medicare Beneficiaries with Diabetes and Incident Prostate Cancer by Metformin Use SEER-Medicare-linked Database 2007- 2010

\begin{tabular}{|c|c|c|c|c|c|c|c|}
\hline & \multicolumn{2}{|c|}{ Overall } & \multicolumn{2}{|c|}{ Metformin Users } & \multicolumn{2}{|c|}{ Non-Metformin User } & \multirow[t]{2}{*}{ Sig. } \\
\hline & $\mathbf{N}$ & $\%$ & $\mathbf{N}$ & $\%$ & $\mathbf{N}$ & $\%$ & \\
\hline All & 2,652 & 100 & 948 & 35.7 & 1,704 & 64.3 & \\
\hline \multicolumn{8}{|c|}{ Predisposing Characteristics } \\
\hline Age at diagnosis, in years & & & & & & & $* * *$ \\
\hline $66-74$ & 1,579 & 59.5 & 609 & 64.2 & 970 & 56.9 & \\
\hline $75+$ & 1,073 & 40.5 & 339 & 35.8 & 734 & 43.1 & \\
\hline Race/ethnicity & & & & & & & * \\
\hline Whites & 1,914 & 72.2 & 680 & 71.7 & 1,234 & 72.4 & \\
\hline African-American & 323 & 12.2 & 108 & 11.4 & 215 & 12.6 & \\
\hline Hispanic/Latino & 150 & 5.7 & 70 & 7.4 & 80 & 4.7 & \\
\hline Others & 265 & 10.0 & 90 & 9.5 & 175 & 10.3 & \\
\hline \multicolumn{8}{|l|}{ Marital status } \\
\hline Unmarried & 222 & 8.4 & 73 & 7.7 & 149 & 8.7 & \\
\hline Married & 1,550 & 58.4 & 581 & 61.3 & 969 & 56.9 & \\
\hline Divorced/Separated & 393 & 14.8 & 136 & 14.3 & 257 & 15.1 & \\
\hline Others & 487 & 18.4 & 158 & 16.7 & 329 & 19.3 & \\
\hline \multicolumn{8}{|c|}{ Enabling Characteristics } \\
\hline Quartile of median census & ome & & & & & & $*$ \\
\hline$\$ 7-\$ 34,522$ & 664 & 25.0 & 265 & 28.0 & 399 & 23.4 & \\
\hline$\$ 34,523-46,224$ & 664 & 25.0 & 243 & 25.6 & 421 & 24.7 & \\
\hline$\$ 46,229-62,764$ & 664 & 25.0 & 229 & 24.2 & 435 & 25.5 & \\
\hline$\$ 62,767-200,008$ & 660 & 24.9 & 211 & 22.3 & 449 & 26.3 & \\
\hline \multicolumn{8}{|c|}{ Quartile of median census 2000 education } \\
\hline $0-8.52$ & 666 & 25.1 & 222 & 23.4 & 444 & 26.1 & \\
\hline $8.53-15.16$ & 655 & 24.7 & 229 & 24.2 & 426 & 25.0 & \\
\hline $15.17-26.09$ & 664 & 25.0 & 248 & 26.2 & 416 & 24.4 & \\
\hline $26.1-100$ & 667 & 25.2 & 249 & 26.3 & 418 & 24.5 & \\
\hline \multicolumn{8}{|l|}{ PSA Screening } \\
\hline Yes & 2,415 & 91.1 & 872 & 92.0 & 1,543 & 90.6 & \\
\hline No & 237 & 8.9 & 76 & 8.0 & 161 & 9.4 & \\
\hline \multicolumn{8}{|l|}{ Visit to a PCP } \\
\hline Yes & 1,858 & 70.1 & 672 & 70.9 & 1,186 & 69.6 & \\
\hline No & 794 & 29.9 & 276 & 29.1 & 518 & 30.4 & \\
\hline Statin Use & & & & & & & $* * *$ \\
\hline Yes & 1,576 & 59.4 & 656 & 69.2 & 920 & 54.0 & \\
\hline No & 1,076 & 40.6 & 292 & 30.8 & 784 & 46.0 & \\
\hline Insulin & & & & & & & $* * *$ \\
\hline Yes & 102 & 3.8 & 62 & 6.5 & 40 & 2.3 & \\
\hline No & 2,550 & 96.2 & 886 & 93.5 & 1,664 & 97.7 & \\
\hline \multicolumn{8}{|c|}{ External-Environment Characteristics } \\
\hline SEER-Regions & & & & & & & $* *$ \\
\hline Northeast & 487 & 18.4 & 146 & 15.4 & 341 & 20.0 & \\
\hline South & 570 & 21.5 & 223 & 23.5 & 347 & 20.4 & \\
\hline North-Central & 351 & 13.2 & 112 & 11.8 & 239 & 14.0 & \\
\hline West & 1,244 & 46.9 & 467 & 49.3 & 777 & 45.6 & \\
\hline \multicolumn{8}{|c|}{ (Continued...) } \\
\hline
\end{tabular}


Table 3. 1

Characteristics of the Study Cohort Elderly Medicare Beneficiaries with Diabetes and Incident Prostate Cancer by Metformin Use SEER-Medicare-linked Database 2007- 2010

\begin{tabular}{|c|c|c|c|c|c|c|c|}
\hline & \multicolumn{2}{|c|}{ Overall } & \multicolumn{2}{|c|}{ Metformin Users } & \multicolumn{2}{|c|}{ Non-Metformin User } & \multirow[t]{2}{*}{ Sig. } \\
\hline & $\mathbf{N}$ & $\%$ & $\mathbf{N}$ & $\%$ & $\mathbf{N}$ & $\%$ & \\
\hline \multicolumn{8}{|c|}{ Need Characteristics } \\
\hline DCSI Quartile & & & & & & & $* *$ \\
\hline 0 to 0 & 846 & 31.9 & 305 & 32.2 & 541 & 31.7 & \\
\hline 1 to 1 & 476 & 17.9 & 195 & 20.6 & 281 & 16.5 & \\
\hline 2 to 3 & 811 & 30.6 & 297 & 31.3 & 514 & 30.2 & \\
\hline 4 to 13 & 519 & 19.6 & 151 & 15.9 & 368 & 21.6 & \\
\hline
\end{tabular}

Notes: Based on the data of 2,652 elderly men aged 66 years and older diagnosed with prostate cancer between 2008 and 2009 using a Surveillance, Epidemiology, and End-Results (SEER)-linked Medicare Part D data. Significant group differences by metformin use are based on Chi-square tests. \% represented in the column are row percentages.

Abbreviations: DCSI: Diabetes Complication Severity Index; PCP: Primary Care Physician; PSA: Prostate Specific Antigen; Sig: Level of Significance;

$* * * \mathrm{p}<.001 ; * * .001 \leq \mathrm{p}<.01 ; * .01 \leq \mathrm{p}<.05$ 
Table 3. 2

Adjusted Odds Ratios and 95\% Confidence Intervals from Logistic Regressions On Metformin Use among Elderly Medicare Beneficiaries with diabetes Incident Prostate Cancer Surveillance, Epidemiology and End Results (SEER)-Medicare Linked Data-2007-2010

\begin{tabular}{|c|c|c|c|}
\hline 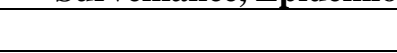 & $\mathrm{AOR}$ & $95 \% \mathrm{CI}$ & Sig. \\
\hline \multicolumn{4}{|c|}{ Predisposing Characteristics } \\
\hline \multicolumn{4}{|l|}{ Age at Diagnosis } \\
\hline 66-74 years & 1.31 & {$[1.10,1.56]$} & $* *$ \\
\hline 75 years or above & Ref & & \\
\hline \multicolumn{4}{|l|}{ Race/ethnicity } \\
\hline Whites & Ref & & \\
\hline African-American & 0.91 & {$[0.69,1.21]$} & \\
\hline Latino & 1.62 & {$[1.12,2.34]$} & ** \\
\hline Others & 0.80 & {$[0.59,1.10]$} & \\
\hline \multicolumn{4}{|l|}{ Marital status } \\
\hline Married & Ref & & \\
\hline Unmarried & 0.81 & {$[0.59,1.11]$} & \\
\hline Divorced/Separated & 0.94 & {$[0.74,1.20]$} & \\
\hline Others & 0.83 & {$[0.66,1.04]$} & \\
\hline \multicolumn{4}{|c|}{ Enabling Characteristics } \\
\hline \multicolumn{4}{|c|}{ Quartile of median census 2000 income } \\
\hline$\$ 7-\$ 34,522$ & 1.72 & {$[1.20,2.46]$} & $* *$ \\
\hline$\$ 34,523-46,224$ & 1.34 & {$[0.97,1.84]$} & \\
\hline$\$ 46,229-62,764$ & 1.18 & {$[0.89,1.56]$} & \\
\hline$\$ 62,767-200,008$ & Ref & & \\
\hline \multicolumn{4}{|c|}{ Quartile of median census 2000 education } \\
\hline $0-8.52$ & 1.24 & {$[0.87,1.77]$} & \\
\hline $8.53-15.16$ & 1.21 & {$[0.90,1.62]$} & \\
\hline $15.17-26.09$ & 1.18 & {$[0.92,1.51]$} & \\
\hline $26.1-100$ & Ref & & \\
\hline \multicolumn{4}{|l|}{ Visit to a PCP } \\
\hline Yes & 1.12 & {$[0.92,1.35]$} & \\
\hline No & Ref & & \\
\hline \multicolumn{4}{|l|}{ Statin Use } \\
\hline Yes & 1.94 & {$[1.63,2.31]$} & $* * *$ \\
\hline No & Ref & & \\
\hline \multicolumn{4}{|l|}{ Insulin } \\
\hline Yes & 2.95 & {$[1.94,4.49]$} & $* * *$ \\
\hline No & Ref & & \\
\hline \multicolumn{4}{|c|}{ Need-Factors } \\
\hline \multicolumn{4}{|l|}{ DCSI Quartile } \\
\hline 0 to 1 & Ref & & \\
\hline 2 to 2 & 1.13 & {$[0.89,1.44]$} & \\
\hline 3 to 3 & 0.98 & {$[0.79,1.21]$} & \\
\hline 4 to 13 & 0.65 & {$[0.51,0.84]$} & $* * *$ \\
\hline
\end{tabular}

External-Environment Characteristics

\begin{tabular}{lcl}
\hline SEER-Regions & & \\
Northeast & Ref & \\
South & 1.40 & {$[1.06,1.85]$} \\
North-Central & 1.07 & {$[0.78,1.47]$} \\
West & 1.37 & {$[1.08,1.75]$}
\end{tabular}

Notes: Based on the data of 2,652 elderly men aged 66 years and older diagnosed with prostate cancer between 2008 and 2009 using a Surveillance, Epidemiology, and End-Results (SEER)-linked Medicare Part D data. Significant group differences are based on log-likelihood test for metformin use.

Abbreviations: DCSI: Diabetes Complication Severity Index; PCP: Primary Care Physician; Ref: Reference group; Sig: Level of Significance;

$* * * \mathrm{p}<.001 ; * * .001 \leq \mathrm{p}<.01 ; * .01 \leq \mathrm{p}<.05$ 


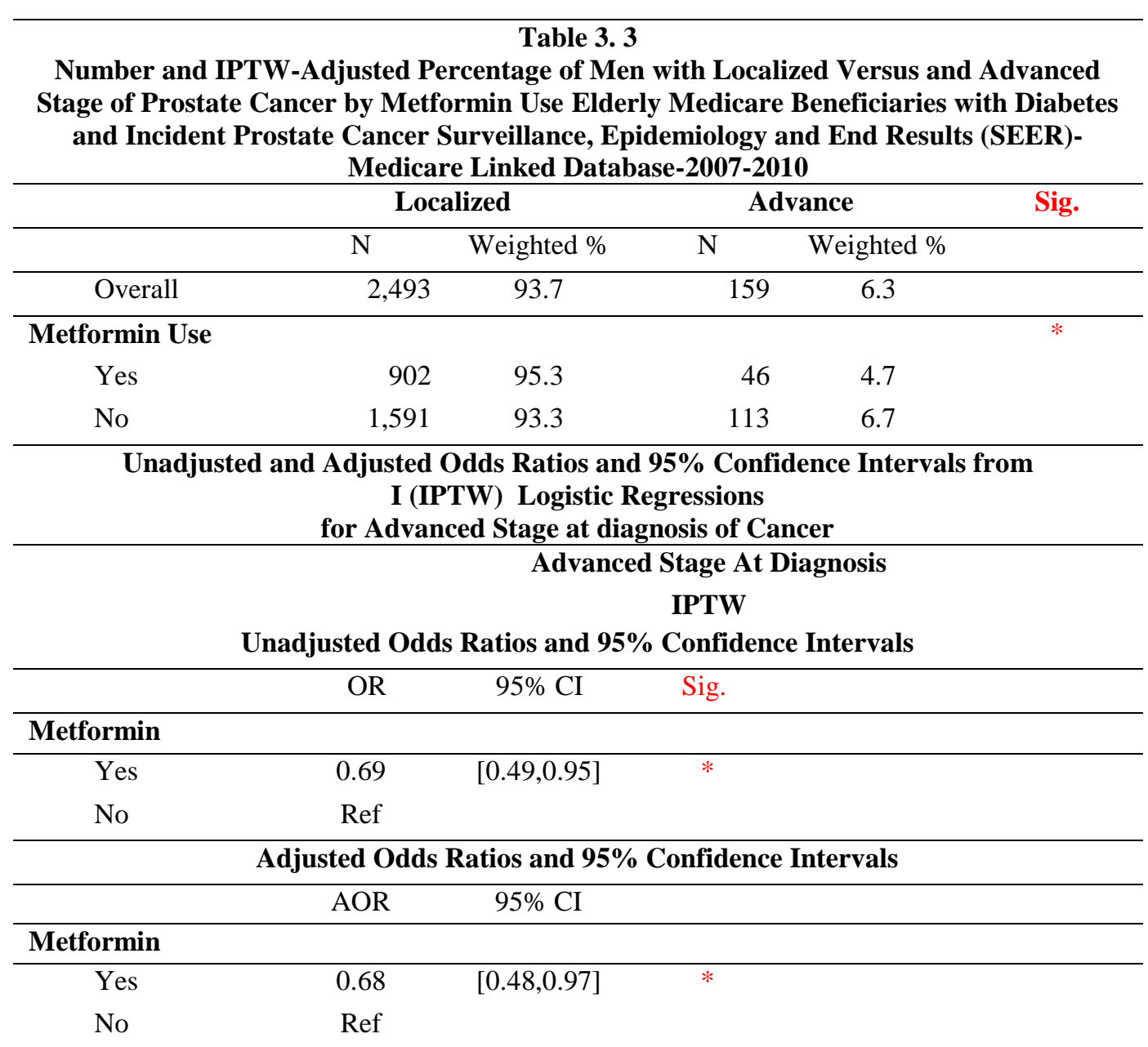

Notes: Based on the data of 2,652 elderly men aged 66 years and older diagnosed with prostate cancer between 2008 and 2009 using a Surveillance, Epidemiology, and End-Results (SEER)-linked Medicare data. \% are weighted percentage for IPTW. Significant difference are based on the log-likelihood test using a logistic regression with IPTW weights. Adjusted model controlled for predisposing, enabling, need and external-environment related factors.

Abbreviations: IPTW: Inverse Probabilities Treatment Weights; PSA: Prostate Specific Antigen Level; PCP; Primary Care Physician Visit Sig: Level of Significance; *** $\mathrm{p}<.001 ; * * .001 \leq \mathrm{p}<.01 ; * .01 \leq \mathrm{p}<.05$ 
Table 3. 4

Numbers and IPTW-Adjusted Percentages of the Initial Cancer Treatment Categories

By Metformin Use Elderly Medicare Beneficiaries with Diabetes and Incident Prostate Cancer

Surveillance, Epidemiology and End Results (SEER)-Medicare Linked Database-2007-2010

Initial Cancer Treatment

\begin{tabular}{|c|c|c|c|c|c|c|c|c|c|}
\hline & \multicolumn{2}{|c|}{$\mathbf{R P}$} & \multicolumn{2}{|c|}{ RT } & \multicolumn{2}{|c|}{ Hormone only } & \multicolumn{2}{|c|}{ None } & \multirow[t]{2}{*}{ Sig. } \\
\hline & $\mathbf{N}$ & Wt \% & $\mathbf{N}$ & Wt \% & $\mathbf{N}$ & $\begin{array}{l}\mathrm{Wt} \\
\%\end{array}$ & $\mathbf{N}$ & Wt. \% & \\
\hline All & 503 & 17.4 & 1,273 & 46.5 & 214 & 8.6 & 662 & 27.6 & \\
\hline Metformin Use & & & & & & & & & $* *$ \\
\hline Yes & 211 & 21.3 & 457 & 46.6 & 71 & 8.6 & 209 & 23.8 & \\
\hline No & 292 & 16.3 & 816 & 46.4 & 143 & 8.7 & 453 & 28.5 & \\
\hline
\end{tabular}

Unadjusted and Adjusted Odds Ratios and 95\% Confidence Intervals (CI) from

IPTW-adjusted Multinomial Logistic Regressions on Initial Cancer Treatment

(Reference Group = No Cancer Treatment)

\begin{tabular}{|c|c|c|c|c|c|c|c|c|c|}
\hline \multicolumn{10}{|c|}{ Unadjusted Odds Ratios and 95\% Confidence Intervals } \\
\hline & \multicolumn{3}{|c|}{$\mathbf{R P}$} & \multicolumn{3}{|c|}{ RT } & \multicolumn{3}{|c|}{ Hormone Therapy } \\
\hline & OR & $95 \% \mathrm{CI}$ & Sig. & OR & $95 \% \mathrm{CI}$ & Sig. & OR & $95 \% \mathrm{CI}$ & Sig. \\
\hline \multicolumn{10}{|c|}{ Metformin Use } \\
\hline Yes & 1.54 & {$[1.24,1.92]$} & *** & 1.20 & {$[1.04,1.44]$} & & 1.18 & {$[0.89,1.56]$} & \\
\hline No & Ref & & & Ref & & & Ref & & \\
\hline
\end{tabular}

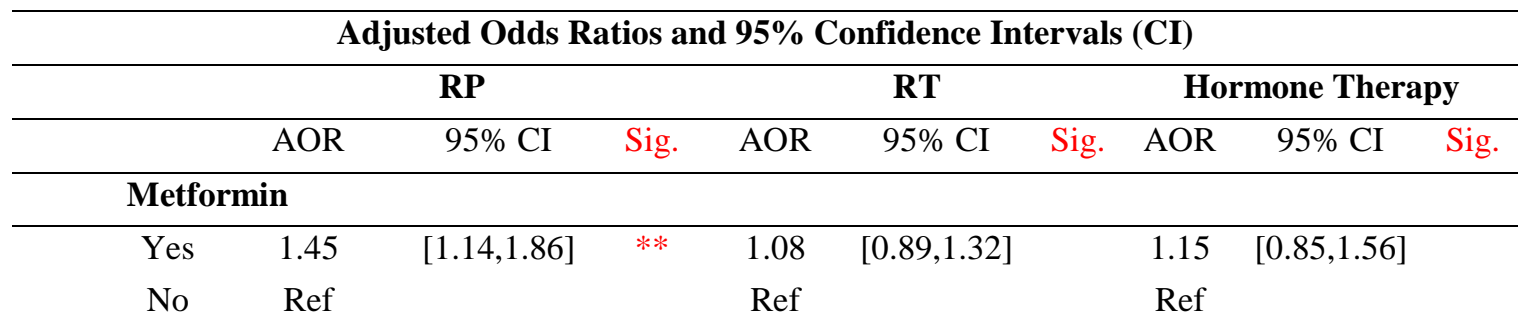

Notes: Based on the data of 2,652 elderly men aged 66 years and older diagnosed with prostate cancer between 2008 and 2009 using a Surveillance, Epidemiology, and End-Results (SEER)-linked Medicare Part D data. Significant group differences by metformin use are based on Chi-square tests. \% represented in the column are weighted percentages. Adjusted model controlled for predisposing, enabling, need and external-environment related factors.

Abbreviations: IPTW: Inverse-Probability Treatment Weights; RP: Radical Prostatectomy; RT: Radiation Therapy; Sig: Level of Significance, Wt: Weighted percentage.

$* * * \mathrm{p}<.001 ; * * .001 \leq \mathrm{p}<.01 ; * .01 \leq \mathrm{p}<.05$ 
Appendix 3. 1 Study Cohort Development Flow diagram for Study Population of Elderly Medicare Beneficiaries diagnosed with Prostate Cancer and Diabetes

Men with prostate cancer identified using SEER site recode: 54 and ICD-10-Code: C61.9) between 2008 and 2009

$$
\mathrm{N}=74,791
$$

Men diagnosed with prostate cancer as a primary cancer and alive at the time of diagnosis

\section{Reason for exclusion:}

- Men diagnosed with prostate cancer at the time of death or autopsy $(\mathrm{N}=644)$

- Men with multiple cancers $(\mathrm{N}=7,119)$

Reasons for exclusion:

- Men with carcinoma in-situ $(\mathrm{N}=17)$

- Men diagnosed with prostate cancer at age 65 or below $(\mathrm{N}=19,523)$

- Invalid death status $(\mathrm{N}=1,870)$

Elderly men diagnosed with prostate cancer as primary cancer, and alive at the time of diagnosis between 2008 and 2008

$$
\mathrm{N}=45,618
$$

Elderly Men diagnosed with prostate cancer as primary cancer at age 66 years and above, malignant tumor, alive at the time of diagnosis and diagnosed between 2002 to 2009

$$
\mathrm{N}=8,442
$$

Elderly Men diagnosed with prostate cancer as primary cancer at age 66 years and above, malignant tumor, alive at the time of diagnosis and diagnosed between 2002 and 2009

With 13-month continuous eligibility in Medicare Part A and Part B and No HMO enrollment during 1 year before diagnosis

$$
\mathrm{N}=\mathbf{2 , 6 5 2}
$$

- No Part A and B enrollment or HMO enrollment $(\mathrm{N}=18,790)$

- No Part D enrollment $(\mathrm{N}=18,386)$

Reasons for exclusion:

- $\quad$ No History of Diabetes $(\mathrm{N}=5,685)$ 


\section{Appendix 3. 2}

Codes and Algorithms to Identify Diabetes Severity Diabetes Complication Severity Index (DCSI) developed by Young et al. and modified by Chang $e t$ al.

\section{Complications ICD-9 CM Code}

DCSI Score

\section{Retinopathy}

Diabetic ophthalmologic disease

$250.5 \mathrm{x}$

Background retinopathy

362.01

\section{1}

Other retinopathy

362.1

Retinal edema

362.83

CSME

Other rerinal disorders

362.53

$362.81,362.82$

362.02

361.xx

369.xx.00-.99

379.23

Proliferative retinopathy

Retinal detachment

Blindness

Vitreous hemorrhage

\section{Nephropathy}

Diabetic nephropathy

Acute glomerulonephritis

250.4

580

581

Nephrotic syndrome

581.81

582

Chronic glomerulonephritis

Nephritis/nephropathy

585

Chronic renal failure

586

Renal failure NOS

593.9

\section{Neuropathy}

Diabetic nephropathy

356.9, $250.6 \quad 1$

Amyotrophy

358.1

Cranial nerve palsy

951.0, $951.1,951.3 \quad 1$

Mononeuropathy

354.0-355.9 1

Charcot's arthopathy

Polyneuropathy

357.2

\section{Cerebrovascular}

TIA

435

$431,433,434,436$

1

Stroke

\section{Cardiovascular}

Atherosclerosis

Other IHD

Angina pectoris

Other chronic IHD

Ventricular fibrillation, arrest

Atrial fibrillation, arrest 


\section{Appendix 3. 2}

Codes and Algorithms to Identify Diabetes Severity Diabetes Complication Severity Index (DCSI)

developed by Young et al. and modified by Chang $e t$ al.

\section{Complications ICD-9 CM Code}

DCSI Score

Heart failure

Atherosclerosis, severe

Aortic aneurysm/dissection

\section{Peripheral vascular disease}

Diabetic PVD

Other aneurysm, LE

PVD

Foot would + complication

Claudication, intermittent

Embolism/thrombosis (LE)

Gangrene

Gas gangrene

Ulcer of lower limbs

\section{Metabolic}

Ketoacidosis

Hyperosmolar

Other coma

\section{8}

$440.23,440.24$

441

443.81, 443.9

892.1

443.9

444.22

785.4

0.4

707.1

250.1

250.2

250.3
2

2

2

1

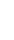

1

1

1

1

2

2

2

2

2

2

1

Note: The table is adapted from the previous algorithm defined by Young et al. and modified by Change et al. to identify the severity of diabetes using claims database. Severity index was based on a scale ranging from 0 to 2 for each complication as follows: $0=$ no abnormality, $1=$ some abnormality, $2=$ severe abnormality.

Abbreviations: ASCVD, atherosclerotic cardiovascular disease; CSME, cystoid macular edema/degeneration; DCSI, Diabetes Complications Severity Index; IHD, ischemic heart disease; ICD-9-CM, International Classification of Diseases, Ninth Revision, Clinical Modification; LE, lower extremity; NOS, not otherwise specified; PVD, peripheral vascular disease; TIA, transient ischemic attack. 


\section{Chapter 4 \\ Impact of Prostate Cancer Diagnosis on Non-Cancer Hospitalizations by Types of Chronic Conditions among Elderly Medicare Beneficiaries with Incident Prostate Cancer}

\subsection{ABSTRACT}

OBJECTIVES: To analyze the impact of cancer diagnosis on non-cancer hospitalizations by comparing them between the pre- and post-cancer period in a cohort of fee-for-service Medicare beneficiaries with incident prostate cancer. The study additionally examined the impact of cancer diagnosis on non-cancer hospitalizations by the types of pre-existing chronic conditions, after controlling for the predisposing, enabling, need, and external environment factors.

METHODS: A population-based retrospective cohort study was conducted using the Surveillance, Epidemiology and End-Results (SEER) -Medicare linked database for the years 2000 to 2010. The study cohort consisted of 57,489 elderly men ( $\geq 67$ years) with incident prostate cancer. Non-cancer hospitalizations were identified in six time periods $\left(\mathrm{t}_{1}-\mathrm{t}_{6}\right)$ before and after the incidence of prostate cancer. Each time period consisted of 120 days. Time periods 1, 2, and 3 represented the pre-cancer period, and time periods 4,5 , and 6 represented the post-cancer period. For each time period, non-cancer hospitalizations were defined as inpatient admissions with primary diagnosis codes not related to prostate cancer.

The types of chronic conditions were grouped into the following seven mutually exclusive categories: (1) only cardio-metabolic conditions; (2) only mental health conditions; (3) only respiratory conditions; (4) cardio-metabolic and mental health conditions; (5) cardio-metabolic and respiratory conditions; (6) all three-cardio-metabolic, mental health, and respiratory conditions, and 7) none of the three types of chronic conditions. Individuals who were diagnosed with new chronic conditions during the pre- and post-cancer periods were excluded. Selection of other independent variables was based on the $\mathrm{ABM}$ model and consisted of the predisposing (age, race/ethnicity, and marital status), enabling (income, education, cancer-treatment, and primary care visit), need (number of other chronic conditions), and external environment (region, number of oncology and urology unit in a county) factors. 
Bivariate and multivariate comparisons of the rates of non-cancer hospitalizations between the preand post-cancer period accounted for repeated measures design. Multivariable regressions were performed using the generalized estimating equation (GEE) technique with logit link function. The extent to which the pre- and post-cancer period non-cancer hospitalizations differed by the types of chronic conditions, after controlling for other independent variables, are reported in terms of the estimated probabilities from the GEE models.

RESULTS: The rate of non-cancer hospitalizations during the post-cancer period was higher as compared to the pre-cancer period. For example, the rates were $9.2 \%$ during the post-cancer period, $\mathrm{t}_{4}$ (i.e. 120 days immediately after the diagnosis of incident cancer) and 3.8\% during the pre-cancer period, an increase of 5.4 percentage points. After accounting for correlations due to repeated measures and unadjusted model, elderly men were $77 \%$ more likely to have any non-cancer hospitalizations during the post-cancer period as compared to the pre-cancer period (Odds Ratio, OR: 1.77, 95\% Confidence Interval, CI: 1.71, 1.82). After adjusting for predisposing, enabling, need and external-environment-related factors, elderly men were $80 \%$ more likely to have non-cancer hospitalizations during the post-cancer period as compared to pre-cancer period (Adjusted OR, AOR: 1.80, 95\% CI: 1.75, 1.86).

For all categories of chronic conditions, the rates of non-cancer related hospitalizations were higher during the post-cancer period as compared to the pre-cancer period. For example, among elderly men with all the three types of chronic conditions, the rates were $26.5 \%$ during the post-cancer period $\left(\mathrm{t}_{4}\right)$ and $15.1 \%$ during the pre-cancer period $\left(\mathrm{t}_{1}\right)$, an increase of 11.4 percentage points. Among elderly men with none of the chronic conditions, the rates were $5.8 \%$ during the post-cancer period $\left(\mathrm{t}_{4}\right)$ and $1.4 \%$ during the pre-cancer period $\left(\mathrm{t}_{1}\right)$, an increase of 4.4 percentage points. . After adjusting for correlations due to repeated measures, elderly men with all the three types of chronic conditions had the greatest increase in the rates of non-cancer hospitalizations during the post-cancer period as compared to the pre-cancer period. Elderly men with none of the three types of chronic conditions had the lowest increase in the rates of noncancer hospitalizations in the post-cancer period as compared to the pre-cancer period.

CONCLUSIONS: Elderly men with prostate cancer had a significant increase in the risk of non-cancer hospitalizations after the diagnosis of prostate cancer. For all chronic condition categories, there was an 
increase in non-cancer hospitalizations during the post-cancer period as compared to the pre-cancer period. Elderly men with all the three types of chronic conditions had the greatest increase in non-cancer hospitalizations compared to all other chronic condition categories.

IMPLICATIONS: The study findings highlight the negative impact of cancer diagnosis on the risk for non-cancer hospitalizations among men with prostate cancer pre-existing chronic conditions. The risk for non-cancer hospitalizations was the highest among those with pre-existing chronic conditions that span multiple organ systems. Future studies need to evaluate whether appropriate management of the preexisting conditions can reduce the risk of non-cancer hospitalization during the post-cancer period. 


\subsection{INTRODUCTION}

The period immediately following a cancer diagnosis can be considered as a period of turbulence for the management of pre-existing chronic conditions. As pointed out by Piette et al., cancer is often considered as a dominant condition that eclipses the management of all other chronic conditions (1). It has been reported that among individuals with cancer, the management of conditions that are not related to cancer may be neglected (2). Undermining the management of chronic conditions among individuals with any cancer may result in adverse outcomes and poor quality of care for non-cancer related conditions. One study among elderly men with prostate cancer found that men with prostate cancer received poor-quality of non-cancer management (3). However, this study did not examine the relationship between non-cancer related hospitalizations and pre-existing chronic conditions.

It is important to examine hospitalizations among elderly men with prostate cancer as they indicate a worsening of existing conditions to the point of requiring immediate attention for monitoring and management in an inpatient environment. In addition, hospitalizations have been associated with significant mortality and healthcare costs (4). In 2012, \$892.4 billion was spent on hospitalizations in the US and hospitalization expenditures were expected to increase by 5\% every year (5). Therefore, the rate of non-cancer hospitalizations among the elderly with prostate cancer not only reflect the hospitalizations for non-cancer related conditions, but also reflect enormous financial burden to the US healthcare system.

Among men of all ages with prostate cancer, many hospitalizations are attributable to chronic conditions other than prostate cancer. For example, using the encounter-level data for the years 1997 through 2004, Milenkovic et al. found that among adults with prostate cancer, $84 \%$ of the inpatient encounters were for chronic conditions other than prostate cancer (6). An overwhelming majority of these inpatient encounters (90\%) was observed among elderly men aged 65 years or older. However, the study investigators did not provide any information on the impact of cancer diagnosis on non-cancer hospitalizations. Furthermore, the study used encounter-level data rather than patient-level data ignoring multiple hospitalizations by the same individual. Thus, the impact of prostate cancer diagnosis on the hospitalizations due to conditions other than cancer remains unknown. 
The initial treatment of prostate cancer may also increase the risk of hospitalizations among elderly men with prostate cancer. Many observational studies have shown a positive association between use of androgen deprivation therapy (ADT) or radiation therapy (RT) and hospitalizations for cardiometabolic conditions among men with prostate cancer (7-9), (10). However, these studies included a population with established prostate cancer and examined the risk of hospitalizations only for specific noncancer hospitalizations. Furthermore, these studies did not investigate the association between cancer diagnosis and non-cancer hospitalizations.

It is important to analyze the association between cancer diagnosis and the risk for non-cancer hospitalizations by types of chronic conditions. Elderly men with prostate cancer have differential rates of chronic conditions. For example, cardio-metabolic conditions (example: heart disease and diabetes) are observed in $18.1 \%$ of men with prostate cancer, followed by respiratory conditions (example: chronic obstructive pulmonary disease-9.8\%) (11) and mental health conditions (example: depression-8\%) (12). Thus, the risk for hospitalizations may differ by the types of chronic conditions. One study, not specific to prostate cancer, reported that individuals with cardio-metabolic conditions had higher rates of annual hospitalizations (40\%) as compared to those with respiratory conditions (20\%) among adults aged 50 years and older in the US (13). The incidence of prostate cancer can have varying impact on the risk for noncancer hospitalizations among men different types of pre-existing chronic conditions. Therefore, it is important to analyze the impact of cancer diagnosis on the risk of non-cancer hospitalizations by the types of pre-existing chronic conditions among elderly men diagnosed with prostate cancer.

An understanding of the risk of non-cancer hospitalization risk after the diagnosis of cancer for those with incident prostate cancer can inform surveillance efforts and the management of chronic conditions among elderly men with prostate cancer. Therefore, the primary objectives of the current study are to analyze the impact of cancer diagnosis on non-cancer hospitalizations among elderly men with incident prostate cancer and examine whether the impact of cancer diagnosis on non-cancer hospitalizations differs by the types of chronic conditions. 


\subsection{CONCEPTUAL FRAMEWORK}

The conceptual framework was based the ABM model, described in Chapter 1. The independent variables for the current study are depicted in the Figure 4.1.

Figure 4. 1 Study Theoretical Framework: Adaptation of Andersen Behavioral Model for Aim 3

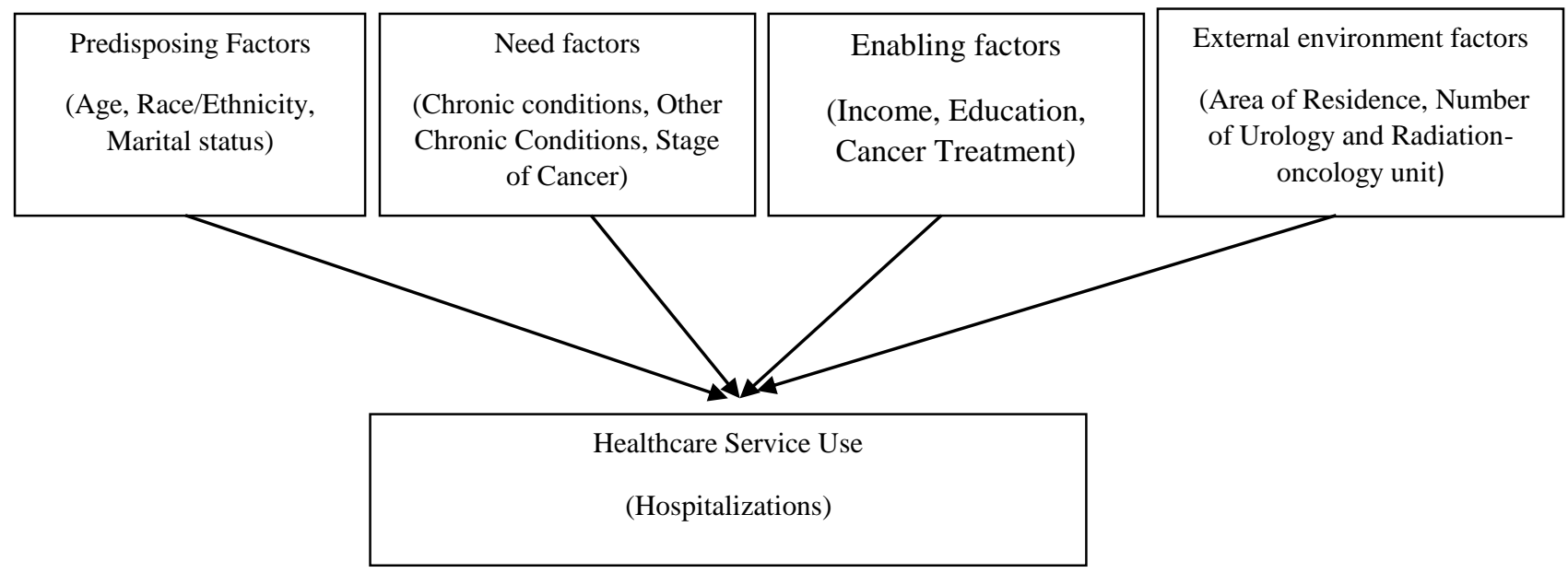

\subsection{METHODS}

\subsubsection{Study Design}

A retrospective longitudinal cohort design with baseline, pre and post-cancer periods was used. Using the date of cancer diagnosis as the index date, a 24-month window before the index date was constructed. This 24-month period was split into two 12-month periods (i.e. the baseline and pre-cancer period). This was necessary because identification of pre-existing chronic conditions and non-cancer hospitalizations during the pre-cancer period involved the use of inpatient claims. To avoid circular reasoning, the first 12-month period was used as the baseline period, during which the types of chronic conditions and other independent variables were measured. The 12-month period before the diagnosis of cancer was considered as the pre-cancer period and this period was used to derive non-cancer hospitalizations. The post-cancer period consisted of 12 months after the diagnosis of cancer. However, to ensure a robust study design, the non-cancer hospitalizations were measured repeatedly every 120 days during the pre- and post-cancer period, yielding a total 6 repeated measures per individual. Thus, $\mathrm{t}_{1}, \mathrm{t}_{2}, \mathrm{t}_{3}$ represented the pre-cancer period and $t_{4}, t_{5}$, and $t_{6}$ represented the post-cancer period. The graphical representation of the study design is shown in Figure 4.2. 
Figure 4. 2 Study Design for Aim 3

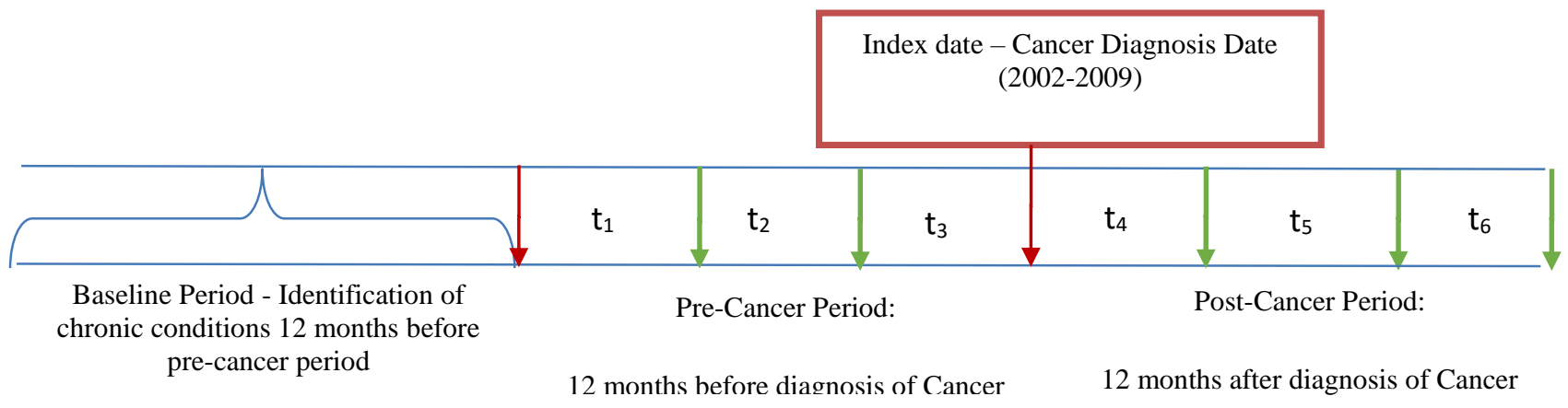

\subsubsection{Data Sources}

The current study utilized the SEER-Medicare linked database with prostate cancer cases diagnosed between 2002 and 2009 and their linked claims between 2000 and 2010. Please see Chapter 1 for details.

\subsubsection{Study Cohort}

The study population was based on 289,701 men diagnosed with incident prostate cancer between January $1^{\text {st }} 2002$ and December $31^{\text {st }} 2009$. Men with multiple cancers $(\mathrm{N}=25,785)$; diagnosed with prostate cancer during the autopsy $(\mathrm{N}=2,944)$; men younger than 67 years of age and died during the study period $(\mathrm{N}=111,643)$ were excluded. The cohort was further restricted to those with continuous feefor-service Medicare Part A and Part B enrollment during the entire study period ( $\mathrm{N}=101,302)$. To reduce misclassification bias, individuals with newly diagnosed conditions during the pre-cancer and post-cancer periods $(\mathrm{N}=40,544)$ were excluded. After excluding individuals with missing information on race/ethnicity, county, and in-situ, unknown, and advanced cancer stage, the final study cohort comprised of 57,589 elderly men with incident prostate cancer. Appendix 4.1 provides details of the study cohort development.

\subsubsection{Key Dependent Variable: Non-cancer hospitalizations}

Inpatient admissions during the pre- and post-cancer periods were derived from the MEDPAR file. Non-cancer hospitalization was defined as any admission to an inpatient facility with a principal diagnosis for conditions other than prostate cancer. Hospitalizations with the primary diagnosis of prostate cancer 
(ICD-9 CM codes - 185, 233.4, or V1046) were considered as prostate-cancer-related hospitalizations and were excluded from the analysis (6). This approach is commonly used in published studies to identify disease-specific hospitalizations $(4,14)$.

However, the challenges in measuring non-cancer hospitalizations should also be noted. Elderly men with prostate cancer may have hospitalizations due to cancer-related complications such as bowel, sexual or urinary dysfunction or cancer-related procedures such as surgery. Therefore, a sensitivity analysis was carried out by excluding hospitalizations for cancer-related complications or primary or secondary procedure codes for cancer-related procedures. As the findings remained consistent between the two definitions, only the results from the primary analyses are reported.

\subsubsection{Key Independent Variable: Types of co-occurring chronic condition (One of the need factors)}

We classified the common types of chronic conditions using the organ domains (15). We selected this approach because of the synergism in treatment and self-management approaches. For example, the management of cardio-metabolic conditions such as diabetes and heart disease have similar treatment and self-management strategies. Among men with prostate cancer, cardio-metabolic diseases (diabetes and heart disease), respiratory diseases (COPD and asthma), and mental health conditions (depression and other mental conditions) are common. These common chronic conditions were classified into seven mutually exclusive categories: (1) cardio-metabolic conditions only; (2) mental health conditions only; (3) respiratory conditions only; (4) cardio-metabolic and mental health conditions; (5) cardio-metabolic and respiratory conditions; (6) having all the three types of chronic conditions; and 7) having none of the three types of chronic conditions. Due to insufficient numbers for the category "respiratory and mental health conditions", this category was excluded from analysis. All the chronic conditions were identified during the baseline period using one inpatient claim or two outpatient claims for each of the chronic conditions.

\subsubsection{Other Independent Variables}

\subsubsection{Predisposing Characteristics}

Age, race/ethnicity, and marital status were the predisposing characteristics. Age at the time of diagnosis (67 to 74 years, 75 and above years), race/ethnicity (White, African American, Hispanic, and 
other), and marital status (married, divorced/separated, unmarried, and others) were identified from the PEDSF file.

\subsubsection{Enabling Characteristics}

Income, education, access to care and the initial cancer treatment were the enabling factors. The PEDSF file provides 2000 census-tract level information as education level and median income. For our population, we created quartiles of census tract education levels and median income. Access to care was defined using proxy measures in the absence of any direct measures for the same. The proxy measure for access to care was measure with at least one visit to a primary care provider during the baseline period (16).

Receipt of active treatment was identified using inpatient, outpatient and carrier files with appropriate ICD-9 CM diagnostic and procedure codes, CPT or HCPCS codes or Revenue Center code during the six months after the index date (Appendix 2.2). Based on previously validated codes, active treatment was either the receipt of radiation, surgery, or hormonal therapy $(17,18)$. Use of the combination of Luteinizing Hormone Releasing hormone (LHRH) agonist and an anti-androgen during the fourth time period after diagnosis of prostate cancer from inpatient or outpatient or carrier file was labeled as hormone therapy (19). Men who did not have any of the above-mentioned treatment categories were classified as having no cancer treatment. We classified the cancer treatment into four groups based on a hierarchy: 1) Radical Prostatectomy (RP); 2) Radiation Therapy (RT); 3) Hormone Therapy; and 4) None of the RP, RT or hormone therapy.

\subsubsection{External environment characteristics}

External environment characteristics comprised of the individual's region (Northeast, South, North-Central, and West) and county-level healthcare resources such number of radiation-oncology units and urology units. (20). County-level healthcare resources were captured by the number of radiation oncology units and urology units were derived from the AHRF. The description of the AHRF file can be found in chapter 1 in method section. 


\section{Need Characteristics:}

A very small percentage of men $(<5 \%)$ had chronic conditions other than the cardio-metabolic, mental-health, and respiratory conditions. Forty-two other conditions were derived using the list of chronic conditions from published literature (1). We calculated the total number of other chronic conditions during the baseline period and categorized as "zero or one condition" and "two or more conditions".

The American Joint Committee on Cancer (AJCC) Tumor-Node-Metastases (TNM) stage classifications was utilized for staging of prostate cancer. The cohort was restricted to elderly men with no-node positive or non-metastatic prostate cancer. Three groups of cancer stage at diagnosis were created as: 1) $\mathrm{T} 1$ or less; 2) $\mathrm{T} 2$ and 3) $\mathrm{T} 3$ or $\mathrm{T} 4$.

\subsection{STATISTICAL ANALYSES}

As the non-cancer hospitalizations were measured repeatedly during each of the six-time periods, the observations are no longer independent and are correlated. The use of standard methods may lead to erroneous variance estimates because these methods assume "independence of observations". Therefore, both the bivariate and multivariate comparisons of the rates of non-cancer hospitalizations between the preand post-cancer period accounted for correlations due to the repeated measures design.

There are many multivariable methods to account for correlations due to repeated measures. One such model is suggested by Liang et al (21). This method involves the use of the generalized estimating equations (GEE) with specific the correlation-matrix. The general form of the model is given in the equation below.

$$
\ln \left(\frac{p_{i j}}{1-p_{i j}}\right)=\beta_{0}+\beta_{1}\left(\text { time }_{i j}\right)+\beta_{2}(C C)_{i}+\cdots+\beta_{n}\left(N_{t h} \text { Variable }_{i}\right.
$$

where $p_{\mathrm{ij}}$ denotes the probability of non-cancer hospitalizations for individual $\mathrm{i}$, in time period $\mathrm{j}$. Time $_{\mathrm{ij}}$ denotes a specific time period $\mathrm{j}$ (pre- or post-cancer period) for an individual, i. $\mathrm{CC}_{\mathrm{i}}$ denotes presence of particular types of chronic conditions at baseline period for an individual, i. The model also included other independent variables such as predisposing, enabling, need and external-environment characteristics measured for an individual, i. $\quad \beta$ s are the regression coefficients to be estimated. As can be 
seen from the equation, except for the pre- and post-cancer time periods, none of the other independent variables varied over time and were considered fixed effects.

The GEE approach developed by Liang and Zeger specifies three types of correlation structures exchangeable, autoregressive, and unstructured -to control for correlations between repeated observations. Exchangeable correlation structure, the most commonly used, assumes that every pair of residuals for a subject have the same correlation. i.e. the same correlation between pairs $\left(t_{1}, t_{3}\right)$ and $\left(t_{4}, t_{6}\right)$ for every individual. The autoregressive correlation structure depends on the time separating two repeated events for the same individual. In other words, the correlation for the non-cancer hospitalizations pair $\left(\mathrm{t}_{1}, \mathrm{t}_{2}\right)$ may be greater than that of the pair $\left(\mathrm{t}_{1}, \mathrm{t}_{4}\right)$. In the unstructured correlation structure, the correlations between the particular pairs of time periods can be different than any other pairs of time periods. As the parameter estimates of the association between non-cancer hospitalization and types of chronic conditions remained the same under different correlation structures (i.e. autoregressive and unstructured), only the results from GEE models with exchangeable correlation structure are reported. The overall model fit was examined by the quasi-likelihood information criterion (qIC) (22).

For ease of interpretation, the predicted probabilities of non-cancer hospitalizations between the pre-and post-cancer period were compared. These probabilities were calculated using the parameter estimates from the GEE models, assuming an additive effect (23). Statistical analyses using GEE models were carried out in STATA version 13. (StataCorp. 2013. Stata Statistical Software: Release 13. College Station, TX: StataCorp LP.) The predicted probabilities were derived using the "margins" command in STATA-13.

It should be noted that in the current study, the term "risk" is often used to describe the results associated with odds ratios (OR). Although the risk ratio and OR will yield different estimates, for events with low prevalence $(\leq 10 \%)$ (24) such as non-cancer hospitalizations, these two measures will produce similar results. 


\subsection{RESULTS}

\subsubsection{Study Population}

The study cohort $(\mathrm{N}=57,489)$ were primarily whites $(82.4 \%)$, in the age group $67-74$ years $(58.3 \%$, mean age $=74.6$ years, standard deviation $=5.1)$, and married $(68.5 \%)$. Most men in the study cohort received treatment for cancer $(80.5 \%)$. (see Table 4.1).

\subsubsection{Non-cancer-Hospitalizations during the pre-and post-cancer period}

Table 4.2 reports the rates of non-cancer-hospitalizations during each of the time periods. The rates of non-cancer-hospitalizations were the highest during the post-cancer period ( $\mathrm{t}_{4}$, i.e., 120 days immediately after the cancer diagnosis) and lowest during the pre-cancer period ( $\left.\mathrm{t}_{2}\right)(9.2 \%$ vs $3.4 \%)$, an increase of 5.8 percentage points.

Table 4.2 also shows the rates of non-cancer hospitalizations by the types of chronic conditions. Of all the categories of chronic conditions, the rates of non-cancer hospitalizations during the post-cancer were significantly higher compared to the pre-cancer period. Among elderly men with all three types of chronic conditions, the rates were $26.5 \%$ during the post-cancer period $\left(\mathrm{t}_{4}\right)$ and $15.1 \%$ during the precancer period $\left(\mathrm{t}_{4}\right)$, an increase of 11.4 percentage points. Among elderly men with none of the chronic conditions, the rates were $5.8 \%$ during the post-cancer period $\left(\mathrm{t}_{4}\right)$ and $1.4 \%$ during the pre-cancer period $\left(t_{1}\right)$, an increase of 4.4 percentage points. Similar increases were seen for other chronic condition categories.

Table 4.3 displays the ORs and AORs and 95\% confidence intervals (CI) using the unadjusted (Model I) and adjusted GEE models (Models II and III) for non-cancer hospitalizations with exchangeable correlation structures. After accounting for the correlations due to repeated measures, model I revealed that elderly men were $77 \%$ more likely to have non-cancer hospitalizations during the post-cancer period as compared to pre-cancer period (OR: $1.77,95 \%$ CI: 1.71, 1.82). In model II, with adjustments for only the types of chronic conditions, elderly men were $79 \%$ more likely to have non-cancer hospitalizations during post-cancer period (AOR: 1.79, 95\% CI: 1.73, 1.84) as compared to the pre-cancer period., In model III, 
which controlled for all the independent variables, elderly men were $80 \%$ more likely to have non-cancer hospitalizations during the post-cancer period as compared to the pre-cancer period.

\subsubsection{Probabilities of Non-cancer hospitalizations by Pre and Post-Cancer Period}

Table 4.4 displays the probabilities of non-cancer hospitalizations by the pre-and post-cancer period for the types of chronic conditions. Based on the fully adjusted model (Model III), an increase in the probability of non-cancer hospitalizations was observed for the post cancer period $(\mathrm{p}=0.028)$ as compared to pre-cancer period (probabilities, p: $0.028 v s .0 .050$, change in $\mathrm{p}=.022$ ).

Elderly men with all the three types of chronic conditions had the greatest increase in the probabilities of non-cancer hospitalizations during post cancer period (p: 0.121) as compared to pre-cancer period (p: 0.159), whereas, elderly men with none of the types of chronic conditions had the lowest change in the probabilities of non-cancer hospitalizations (p: 0.021) during post-cancer period as compared to precancer period (p: 0.028). With regard to other chronic condition categories, those with cardio-metabolic conditions alone, those with cardio-metabolic and respiratory conditions, those with cardio-metabolic and mental-health conditions had higher risk of non-cancer hospitalizations during the post-cancer period and pre-cancer period ( 0.046 vs $0.061,0.073$ vs $0.097,0.067$ vs 0.090$)$, respectively.

\subsection{DISCUSSION}

The current study analyzed the impact of prostate cancer diagnosis on the risk of non-cancer hospitalizations among elderly fee-for-service Medicare beneficiaries with localized incident prostate cancer between 2002 and 2009 using the SEER-Medicare linked database. Till date, the current study is the largest population-based cohort that has examined the risk of non-cancer hospitalizations before and after a diagnosis of prostate cancer among elderly men with incident prostate cancer. We observed many noteworthy findings, which have implications for a better management of pre-existing chronic conditions among men with incident prostate cancer.

Elderly men with an incident prostate cancer had a higher risk of non-cancer hospitalization during the post-cancer period as compared to the pre-cancer period, after controlling for a comprehensive list of risk factors. A closer examination of the rates of hospitalizations over six time periods suggested that the 
highest rates of non-cancer hospitalizations occurred during first four months after the cancer diagnosis. There are several plausible explanations for this increased risk. It has been documented that the diagnosis of prostate cancer, by itself, can trigger psychological distress, anxiety and suicidal ideations (39). The increase in the psychological stress may increase the blood levels of epinephrine and norepinephrine resulting in increased heart rate, blood pressure and levels of blood sugars. In fact, studies have shown that acute psychological stress may lead to stimulate inflammatory markers such as interleukins, sex hormones and cortisol levels, which may precipitate into serious conditions such as heart disease (25). Another plausible explanation is that a diagnosis of cancer may eclipse the management of all other chronic conditions and patient care may be centered on cancer treatment and recovery from cancer.

Upon closer examination of our study findings by types of chronic conditions, we found that those with cardio-metabolic conditions alone or combinations of cardio-metabolic conditions with respiratory or mental health conditions had a greater increase in the risk of non-cancer hospitalizations during the postcancer period as compared to the pre-cancer period. Previous literature suggests that elderly men with preexisting cardio-metabolic conditions may experience acute emotional or psychological stress, which may lead to greater incidence of cardiovascular events $(26,27)$, which in turn may lead to increased hospitalizations. It has to be noted that the current study did not include newly-diagnosed chronic conditions, therefore, increased hospitalizations during the post-cancer period for this group may suggest the worsening of pre-existing cardio-metabolic conditions due to prostate cancer diagnosis.

The study findings reinforce the need for more research on management of non-cancer conditions before and after diagnosis of cancer and its effect on the non-cancer hospitalizations among elderly men with pre-existing chronic conditions, Special attention needs to be paid to chronic disease care given during the period immediately following the cancer diagnosis. As elderly men with cardio-metabolic conditions are most vulnerable to increased hospitalizations, research, program, policy, and intervention efforts need to focus on reducing the excess non-cancer hospitalizations in this group.

The study findings have a key methodological implication for estimating cancer-related healthcare utilization. Typically, cancer-related annual utilization among men with prostate cancer is estimated by subtracting the total utilization during the year before cancer diagnosis from the total utilization after in a 
year after cancer diagnosis with an assumption of no change of non-cancer related utilizations during preand post-cancer period (28-32). The current study findings revealed a greater risk of non-cancer hospitalizations during the year after as compared to the year before the diagnosis of prostate cancer. Therefore, the standard methods of calculating cancer-related utilization may overestimate the cancerrelated utilizations.

Our study has many advantages. The current study is one of the very few studies to explore the trajectory of non-cancer outcomes (i.e. hospitalizations) during the pre-cancer and post-cancer period among fee-for-service Medicare beneficiaries with incident localized prostate cancer. To the best of our knowledge, this study is the largest population-based study, which used a comprehensive list of factors using the cancer registry-linked administrative claims data. This study used a robust repeated measures study design. The pre-existing chronic conditions were classified using a conceptual framework adopted by the Department of Health and Human Services (DHHS) for research, program, and policy perspectives.

However, the current study findings need to be interpreted in the context of its limitations. Although, due diligence was given in measuring non-cancer hospitalizations based on previously published studies, one cannot rule out the possibility that some non-cancer hospitalizations may be due to cancer treatment or cancer-related complications. For example, cancer surgery may have led to complications such as the sepsis or other infections, which were considered as non-cancer hospitalizations in the current study. Despite having a large cohort, cell sizes for some chronic conditions were too small to be included in the analyses.

Furthermore, an observational study design was used, which has its own limitations. The study population was restricted to elderly men enrolled in fee-for-service Medicare services only residing in the SEER-regions, therefore, we cannot generalize the study findings to all elderly Medicare beneficiaries with prostate cancer. Although we controlled for a comprehensive list of variables to examine multivariable associations between the risk of non-cancer hospitalizations and incidence of prostate cancer, we were not able to control for other risk factors such as the body-mass index, physical activity, and smoking, which may be associated with greater risk of non-cancer hospitalizations among elderly men with prostate cancer. 


\subsection{CONCLUSION}

Diagnosis of incident prostate cancer was associated with an increase in the risk of non-cancer hospitalizations among elderly men with incident localized prostate cancer. Specifically, those with cardiometabolic conditions only or those with cardio-metabolic conditions and mental health or respiratory conditions had a higher increase in the risk of non-cancer hospitalizations during the post-cancer period as compared to the pre-cancer period. Future research needs to examine whether better management strategies for cardio-metabolic and other chronic conditions can reduce the increased risk for non-cancer hospitalizations after a diagnosis of prostate cancer. 


\subsection{REFERENCES}

1. Piette JD, Kerr EA. The Impact of Comorbid Chronic Conditions on Diabetes Care. Diabetes Care. 2006 March 01;29(3):725-31.

2. Redelmeier DA, Tan SH, Booth GL. The treatment of unrelated disorders in patients with chronic medical diseases. N Engl J Med. 1998 May 21;338(21):1516-20.

3. Snyder CF, Frick KD, Herbert RJ, Blackford AL, Neville BA, Wolff AC, et al. Quality of care for comorbid conditions during the transition to survivorship: differences between cancer survivors and noncancer controls. J Clin Oncol. 2013 Mar 20;31(9):1140-8.

4. Anhang Price, R. (RAND), Stranges, E. (Thomson Reuters) and Elixhauser, A. (Agency for Healthcare Quality and Research). Cancer Hospitalizations for Adults, 2009. HCUP Statistical Brief \#125. February 2012. Agency for Healthcare Research and Quality, Rockville, MD. http://www.hcupus. ahrq.gov/reports/statbriefs/sb125.pdf .

5. National Health Expenditure Projections 2012-2022 [Internet].; 2014 []. Available from: http://www.cms.gov/Research-Statistics-Data-and-Systems/Statistics-Trends-and-

Reports/NationalHealthExpendData/downloads/proj2012.pdf.

6. Milenkovic, M. (Thomson Medstat), Russo, C. A. (Thomson Medstat), and Elixhauser, A. (AHRQ). Hospital Stays for Prostate Cancer, 2004. HCUP Statistical Brief \#30. May 2007 Agency for Healthcare Research and Quality, Rockville, MD. http://www.hcup-us.ahrq.gov/reports/statbriefs/sb30.pdf.

7. Keating NL, O'Malley AJ, Freedland SJ, Smith MR. Diabetes and cardiovascular disease during androgen deprivation therapy: observational study of veterans with prostate cancer. J Natl Cancer Inst. 2010 Jan 6;102(1):39-46.

8. Saigal CS, Gore JL, Krupski TL, Hanley J, Schonlau M, Litwin MS, et al. Androgen deprivation therapy increases cardiovascular morbidity in men with prostate cancer. Cancer. 2007 Oct 1;110(7):1493-500. 9. Tsai HK, D'Amico AV, Sadetsky N, Chen MH, Carroll PR. Androgen deprivation therapy for localized prostate cancer and the risk of cardiovascular mortality. J Natl Cancer Inst. 2007 Oct 17;99(20):1516-24. 10. Bovelli D, Plataniotis G, Roila F, ESMO Guidelines Working Group. Cardiotoxicity of chemotherapeutic agents and radiotherapy-related heart disease: ESMO Clinical Practice Guidelines. Ann Oncol. 2010 May;21 Suppl 5:v277-82.

11. Edwards BK, Noone AM, Mariotto AB, Simard EP, Boscoe FP, Henley SJ, et al. Annual Report to the Nation on the status of cancer, 1975-2010, featuring prevalence of comorbidity and impact on survival among persons with lung, colorectal, breast, or prostate cancer. Cancer. 2014 May 1;120(9):1290-314. 12. Jayadevappa R, Malkowicz SB, Chhatre S, Johnson JC, Gallo JJ. The burden of depression in prostate cancer. Psychooncology. 2012 Dec;21(12):1338-45.

13. Chronic Care: Call For Action Refor: Chronic Conditions among Older Americans [Internet]. []. Available from: http://assets.aarp.org/rgcenter/health/beyond 50 hor_conditions.pdf.

14. Wang G, Zhang Z, Ayala C, Wall HK, Fang J. Costs of heart failure-related hospitalizations in patients aged 18 to 64 years. Am J Manag Care. 2010 Oct;16(10):769-76.

15. Fortin M, Dubois MF, Hudon C, Soubhi H, Almirall J. Multimorbidity and quality of life: a closer look. Health Qual Life Outcomes. 2007 Aug 6;5:52.

16. Yu JB, Soulos PR, Herrin J, Cramer LD, Potosky AL, Roberts KB, et al. Proton versus intensitymodulated radiotherapy for prostate cancer: patterns of care and early toxicity. J Natl Cancer Inst. 2013 Jan 2;105(1):25-32.

17. Trantham LC, Nielsen ME, Mobley LR, Wheeler SB, Carpenter WR, Biddle AK. Use of prostatespecific antigen testing as a disease surveillance tool following radical prostatectomy. Cancer. 2013 Oct 1;119(19):3523-30.

18. Wong YN, Mitra N, Hudes G, Localio R, Schwartz JS, Wan F, et al. Survival associated with treatment vs observation of localized prostate cancer in elderly men. JAMA. 2006 Dec 13;296(22):2683-93.

19. Shahinian VB, Kuo YF, Freeman JL, Goodwin JS. Determinants of androgen deprivation therapy use for prostate cancer: role of the urologist. J Natl Cancer Inst. 2006 Jun 21;98(12):839-45.

20. Area Resource File: Overview [Internet].; 2014 []. Available from: http://ahrf.hrsa.gov/overview.htm. 21. Liang KY, Zeger SL. Regression analysis for correlated data. Annu Rev Public Health. 1993;14:43-68. 22. Pan W. Akaike's Information Criterion in Generalized Estimating Equations. Biometrics.

2001;57(1):120-5. 
23. Landerman LR, Mustillo SA, Land KC. Modeling Repeated Measures Of Dichotomous Data: Testing Whether the Within-Person Trajectory of Change Varies Across Levels of Between-Person Factors. Soc Sci Res. 2011 Sep 1;40(5):1456-64.

24. Zhang J, Yu KF. What's the relative risk? A method of correcting the odds ratio in cohort studies of common outcomes. JAMA. 1998 Nov 18;280(19):1690-1.

25. Burg MM, Meadows J, Shimbo D, Davidson KW, Schwartz JE, Soufer R. Confluence of depression and acute psychological stress among patients with stable coronary heart disease: effects on myocardial perfusion. J Am Heart Assoc. 2014 Oct 30;3(6):e000898.

26. Wittstein IS, Thiemann DR, Lima JAC, Baughman KL, Schulman SP, Gerstenblith G, et al.

Neurohumoral Features of Myocardial Stunning Due to Sudden Emotional Stress. N Engl J Med. 2005 02/10;2015/05;352(6):539-48.

27. Trichopoulos D, Katsouyanni K, Zavitsanos X, Tzonou A, Dalla-Vorgia P. Psychological stress and fatal heart attack: the Athens (1981) earthquake natural experiment. Lancet. 1983 Feb 26;1(8322):441-4. 28. Sanyal C, Aprikian AG, Chevalier S, Cury FL, Dragomir A. Direct cost for initial management of prostate cancer: a systematic review. Curr Oncol. 2013 Dec;20(6):e522-31.

29. Burkhardt JH, Litwin MS, Rose CM, Correa RJ, Sunshine JH, Hogan C, et al. Comparing the Costs of Radiation Therapy and Radical Prostatectomy for the Initial Treatment of Early-Stage Prostate Cancer. Journal of Clinical Oncology. 2002 June 15;20(12):2869-75.

30. Zeliadt SB, Etzioni R, Ramsey SD, Penson DF, Potosky AL. Trends in treatment costs for localized prostate cancer: the healthy screenee effect. Med Care. 2007 Feb;45(2):154-9.

31. Roehrborn CG, Albertsen P, Stokes ME, Black L, Benedict A. First-year costs of treating prostate cancer: estimates from SEER-Medicare data. Prostate Cancer Prostatic Dis. 2009;12(4):355-60.

32. Penberthy L, Retchin SM, McDonald MK, McClish DK, Desch CE, Riley GF, et al. Predictors of Medicare costs in elderly beneficiaries with breast, colorectal, lung, or prostate cancer. Health Care Manag Sci. 1999 Jul;2(3):149-60. 
Table 4. 1

Characteristics of Elderly Medicare Beneficiaries with Incident Prostate Cancer Surveillance, Epidemiology and End Results (SEER)-

Medicare Linked Database 2000 - 2010

\begin{tabular}{lrc}
\hline & N & \% \\
\hline All & 57,489 & 100 \\
Types of Chronic Conditions & & \\
CM only & 21,712 & 37.8 \\
MH Only & 813 & 1.4 \\
RSP only & 2,277 & 4.0 \\
CM + MH & 1,148 & 2.0 \\
CM + RSP & 5,114 & 8.9 \\
None & 25,811 & 44.9 \\
All Three & 614 & 1.1 \\
\hline
\end{tabular}

Predisposing Characteristics

\begin{tabular}{|c|c|c|}
\hline \\
\hline \multicolumn{3}{|l|}{ Age at diagnosis, in years } \\
\hline $66-74$ & 33,495 & 58.3 \\
\hline $75+$ & 23,994 & 41.7 \\
\hline \multicolumn{3}{|l|}{ Race/ethnicity } \\
\hline Whites & 47,384 & 82.4 \\
\hline African-American & 6,272 & 10.9 \\
\hline Hispanic/Latino & 1,011 & 1.8 \\
\hline Others & 2,822 & 4.9 \\
\hline \multicolumn{3}{|l|}{ Marital status } \\
\hline Unmarried & 3,654 & 6.4 \\
\hline Married & 39,386 & 68.5 \\
\hline Divorced/Separated & 6,993 & 12.2 \\
\hline Others & 7,456 & 13.0 \\
\hline \multicolumn{3}{|c|}{ Enabling Characteristics } \\
\hline \multicolumn{3}{|c|}{ Quartile of median census 2000 income } \\
\hline$\$ 7-\$ 34,522$ & 14,362 & 25.0 \\
\hline$\$ 34,523-46,224$ & 14,368 & 25.0 \\
\hline$\$ 46,229-62,764$ & 14,369 & 25.0 \\
\hline$\$ 62,767-200,008$ & 14,390 & 25.0 \\
\hline \multicolumn{3}{|c|}{ Quartile of median census 2000 education } \\
\hline $0-8.52$ & 14,371 & 25.0 \\
\hline $8.53-15.16$ & 14,376 & 25.0 \\
\hline $15.17-26.09$ & 14,373 & 25.0 \\
\hline $26.1-100$ & 14,369 & 25.0 \\
\hline \multicolumn{3}{|l|}{ Visit to a PCP } \\
\hline Yes & 37,405 & 65.1 \\
\hline No & 20,084 & 34.9 \\
\hline \multicolumn{3}{|l|}{ Active Treatment } \\
\hline $\mathrm{RP}$ & 11,520 & 20.0 \\
\hline RT & 29,234 & 50.9 \\
\hline Hormone Therapy & 5,510 & 9.6 \\
\hline No Treatment & 11,225 & 19.5 \\
\hline \multicolumn{3}{|c|}{ External-Environment Characteristics } \\
\hline \multicolumn{3}{|l|}{ SEER-Regions } \\
\hline Northeast & 11,360 & 19.8 \\
\hline South & 14,095 & 24.5 \\
\hline North-Central & 7,288 & 12.7 \\
\hline West & 24,746 & 43.0 \\
\hline & (Continued & \\
\hline
\end{tabular}




\begin{tabular}{|c|c|c|}
\hline \multicolumn{3}{|c|}{$\begin{array}{c}\text { Table 4. 1 } \\
\text { Characteristics of Elderly Medicare Beneficiaries with Incident Prostate Cancer } \\
\text { Surveillance, Epidemiology and End Results (SEER)- } \\
\text { Medicare Linked Database } 2000-2010\end{array}$} \\
\hline & & $\%$ \\
\hline \multicolumn{3}{|c|}{ Quartile of Radiation Oncology } \\
\hline 0 to 1 & 15,127 & 26.3 \\
\hline 2 to 6 & 13,203 & 23.0 \\
\hline 7 to 22 & 14,739 & 25.6 \\
\hline 23 to 147 & 14,420 & 25.1 \\
\hline \multicolumn{3}{|c|}{ Quartile of Urology Centers } \\
\hline 0 to 3 & 14,107 & 24.5 \\
\hline 4 to 16 & 14,390 & 25.0 \\
\hline 17 to 44 & 14,130 & 24.6 \\
\hline 45 to 343 & 14,862 & 25.9 \\
\hline \multicolumn{3}{|l|}{ Year of Diagnosis } \\
\hline $2002-2005$ & 28,275 & 49.2 \\
\hline 2006-2009 & 29,214 & 50.8 \\
\hline \multicolumn{3}{|c|}{ Need Characteristics } \\
\hline \multicolumn{3}{|c|}{ Number of Other Chronic Conditions } \\
\hline$\leq$ One & 38,435 & 66.9 \\
\hline$>$ One & 19,054 & 33.1 \\
\hline \multicolumn{3}{|l|}{ T-Stage } \\
\hline$\leq \mathrm{T} 1$ & 32,063 & 55.8 \\
\hline $\mathrm{T} 2$ & 23,905 & 41.6 \\
\hline$\geq \mathrm{T} 3$ & 1,521 & 2.6 \\
\hline
\end{tabular}

Notes: Notes: Based on 57,489 elderly men, aged 67 years and older, diagnosed with incident prostate cancer between 2002 and 2009 and alive throughout the observation period.

Abbreviations: CM: Cardio-metabolic conditions; MH: Mental health conditions; PCP: Primary Care Physician; RESP: Respiratory conditions; RP: Radical Prostatectomy; RT: Radiation Therapy 
Table 4. 2

Number and Percentage with Non-Cancer Related Hospitalizations during the Pre- and Post-Cancer Period Elderly Medicare beneficiaries with Incident Prostate Cancer Surveillance, Epidemiology and End Results (SEER)-Medicare Linked Database-2002-2010

\begin{tabular}{|c|c|c|c|c|c|c|c|c|c|c|c|c|}
\hline & \multicolumn{6}{|c|}{ Pre-Cancer Period } & \multicolumn{6}{|c|}{ Post-Cancer Period } \\
\hline & \multicolumn{2}{|c|}{$\mathbf{t}_{1}$} & \multicolumn{2}{|c|}{$\mathbf{t}_{2}$} & \multicolumn{2}{|c|}{$\mathbf{t}_{3}$} & \multicolumn{2}{|c|}{$\mathbf{t}_{4}$} & \multicolumn{2}{|c|}{$\mathbf{t}_{5}$} & \multicolumn{2}{|c|}{$t_{6}$} \\
\hline & $\mathbf{N}$ & $\%$ & $\mathbf{N}$ & $\%$ & $\mathbf{N}$ & $\%$ & $\mathbf{N}$ & $\%$ & $\mathbf{N}$ & $\%$ & $\mathbf{N}$ & $\%$ \\
\hline Overall & 2,202 & 3.8 & 1,967 & 3.4 & 2,244 & 3.9 & 5,297 & 9.2 & 2,835 & 4.9 & 2,875 & 5.0 \\
\hline None *** $^{*}$ & 367 & 1.4 & 357 & 1.4 & 477 & 1.8 & 1,493 & 5.8 & 528 & 2.0 & 553 & 2.1 \\
\hline CM only** & 1,015 & 4.7 & 929 & 4.3 & 992 & 4.6 & 2,308 & 10.6 & 1,331 & 6.1 & 1,322 & 6.1 \\
\hline MH only** & 26 & 3.2 & 23 & 2.8 & 35 & 4.3 & 63 & 7.7 & 33 & 4.1 & 33 & 4.1 \\
\hline $\mathrm{CM}+\mathrm{RESP} * *$ & 499 & 9.8 & 435 & 8.5 & 497 & 9.7 & 874 & 17.1 & 622 & 12.2 & 659 & 12.9 \\
\hline All Three $* * *$ & 120 & 19.5 & 92 & 15.0 & 93 & 15.1 & 163 & 26.5 & 129 & 21.0 & 121 & 19.7 \\
\hline
\end{tabular}

Notes: Based on 57,489 elderly men, aged 67 years and older, diagnosed with incident prostate cancer between 2002 and 2009 and alive throughout the observation period. Significant differences in non-cancer hospitalizations over time were tested with Wald chi-square, after accounting for correlations due to repeated measures. \% represented in the column are column percentage.

Abbreviations: CM: Cardio-metabolic conditions; MH: Mental illness; Q1-Q6: Quarter1 -6; RESP: Respiratory conditions;

$* * * \mathrm{p}<.001 ; * * .001 \leq \mathrm{p}<.01 ; * .01 \leq \mathrm{p}<.05$ 
Table 4. 3

Adjusted Odds Ratios and 95\% Confidence Intervals (CI) of Cancer Diagnosis Period From GEE models on Non-Cancer Related Hospitalizations Elderly Men with Incident Prostate Cancer Surveillance, Epidemiology and End Results (SEER)Medicare Linked Database-2000-2010

\begin{tabular}{|c|c|c|c|}
\hline \multicolumn{4}{|c|}{ Model 1, adjusting for pre-and post-cancer period } \\
\hline & $\mathbf{O R}$ & $95 \% \mathrm{CI}$ & Sig. \\
\hline \multicolumn{4}{|l|}{ Variable } \\
\hline Pre-cancer & Ref & & \\
\hline Post-cancer & 1.77 & {$[1.71,1.82]$} & $* * *$ \\
\hline \multicolumn{4}{|c|}{$\begin{array}{c}\text { Model 2, adjusting for pre- and post-cancer period }+ \text { Types of Chronic } \\
\text { Conditions }\end{array}$} \\
\hline & AOR & $95 \% \mathrm{CI}$ & Sig. \\
\hline Pre-cancer & Ref & & \\
\hline Post-cancer & 1.79 & {$[1.73,1.84]$} & $* * *$ \\
\hline \multicolumn{4}{|c|}{$\begin{array}{c}\text { Model 3, adjusting for pre- and post-cancer period }+ \text { Types of Chronic } \\
\text { Conditions }+ \text { predisposing, enabling, need, and external environment } \\
\text { characteristics }\end{array}$} \\
\hline & AOR & $95 \% \mathrm{CI}$ & Sig. \\
\hline Pre-cancer & Ref & & \\
\hline Post-cancer & 1.80 & {$[1.75,186]$} & $* * *$ \\
\hline
\end{tabular}

Notes: Based on 57,489 elderly men, aged 67 years and older, diagnosed with incident prostate cancer between 2002 and 2009 and alive throughout the observation period. Significant differences are based on the log-likelihood test using a repeated measure generalized estimating equations.

AOR: Adjusted Odds Ratio; GEE: Generalized Estimating Equation; OR: Odds Ratio; Sig: Level of Sig: Significance.

$* * * \mathrm{p}<.001 ; * * .001 \leq \mathrm{p}<.01 ; * .01 \leq \mathrm{p}<.05$ 


\begin{tabular}{|c|c|c|c|c|c|c|}
\hline \multicolumn{7}{|c|}{$\begin{array}{c}\text { Table } 4.4 \\
\text { Predicted Probabilities with 95\% Confidence Intervals for Types of Chronic Conditions From GEE analysis } \\
\text { on Non-Cancer Related Hospitalizations Elderly Medicare Beneficiaries with Incident Prostate Cancer } \\
\text { Surveillance, Epidemiology and End Results (SEER)-Medicare Linked Database-2000-2010 }\end{array}$} \\
\hline & \multicolumn{2}{|c|}{ Pre-cancer } & \multicolumn{2}{|c|}{ Post-cancer } & \multirow{2}{*}{$\begin{array}{l}\text { Changes in } \\
\text { Probabilities }\end{array}$} & \multirow{2}{*}{$\begin{array}{l}\text { Additive } \\
\text { Effect }\end{array}$} \\
\hline & $\mathbf{P}$ & $95 \% \mathrm{CI}$ & $\mathbf{P}$ & $95 \% \mathrm{CI}$ & & \\
\hline \multicolumn{7}{|c|}{ Model 1, adjusted for pre- and post-cancer period } \\
\hline Overall $* * *$ & 0.037 & {$[0.036,0.038]$} & 0.064 & {$[0.063,0.065]$} & 0.027 & \\
\hline \multicolumn{7}{|c|}{ Model 2, adjusted for pre-and post-cancer period + Types of Chronic Conditions } \\
\hline Overall $* * *$ & 0.031 & {$[0.030,0.031]$} & 0.055 & {$[0.054,0.056]$} & 0.024 & \\
\hline \multicolumn{7}{|c|}{ Types of Chronic Conditions $* * *$} \\
\hline None & 0.018 & {$[0.017,0.018]$} & 0.031 & {$[0.030,0.032]$} & 0.013 & Ref \\
\hline CM only & 0.044 & {$[0.043,0.046]$} & 0.077 & {$[0.075,0.079]$} & 0.033 & 0.020 \\
\hline MH only & 0.032 & {$[0.027,0.037]$} & 0.055 & {$[0.047,0.063]$} & 0.023 & 0.010 \\
\hline RESP only & 0.029 & {$[0.026,0.031]$} & 0.05 & {$[0.045,0.054]$} & 0.021 & 0.008 \\
\hline $\mathrm{CM}+\mathrm{MH}$ & 0.075 & {$[0.069,0.081]$} & 0.127 & {$[0.117,0.137]$} & 0.052 & 0.039 \\
\hline $\mathrm{CM}+\mathrm{RESP}$ & 0.087 & {$[0.084,0.091]$} & 0.146 & {$[0.141,0.151]$} & 0.059 & 0.046 \\
\hline All Three & 0.150 & {$[0.138,0.162]$} & 0.239 & {$[0.222,0.256]$} & 0.089 & 0.076 \\
\hline \multicolumn{7}{|c|}{$\begin{array}{c}\text { Model } 3 \text { adjusted for pre- and post-cancer period }+ \text { Types of Chronic Conditions + predisposing, enabling, need, } \\
\text { and external environment characteristics }\end{array}$} \\
\hline Overall $* * *$ & 0.028 & {$[0.027,0.029]$} & 0.050 & {$[0.049,0.051]$} & 0.022 & \\
\hline \multicolumn{7}{|c|}{ Types of Chronic Conditions $* * *$} \\
\hline None & 0.017 & {$[0.017,0.018]$} & 0.031 & {$[0.030,0.032]$} & 0.014 & Ref \\
\hline CM only & 0.039 & {$[0.038,0.041]$} & 0.069 & {$[0.067,0.071]$} & 0.030 & 0.016 \\
\hline MH only & 0.027 & {$[0.023,0.031]$} & 0.048 & {$[0.041,0.055]$} & 0.021 & 0.007 \\
\hline RESP only & 0.022 & {$[0.020,0.024]$} & 0.039 & {$[0.035,0.043]$} & 0.017 & 0.003 \\
\hline $\mathrm{CM}+\mathrm{MH}$ & 0.056 & {$[0.051,0.061]$} & 0.097 & {$[0.089,0.105]$} & 0.041 & 0.027 \\
\hline $\mathrm{CM}+\mathrm{RESP}$ & 0.063 & {$[0.061,0.065]$} & 0.108 & {$[0.103,0.112]$} & 0.045 & 0.031 \\
\hline All Three & 0.103 & {$[0.095,0.115]$} & 0.172 & {$[0.159,0.187]$} & 0.069 & 0.055 \\
\hline
\end{tabular}

Notes: Based on 57,489 elderly men, aged 67 years and older, diagnosed with incident prostate cancer between 2002 and 2009 and alive throughout the observation period. Significant differences are based on the log-likelihood test using a repeated measure generalized estimating equations.

Abbreviations: CM: Cardio-metabolic conditions; MH: Mental health conditions; RESP: Respiratory conditions; Sig: Level of Significance;

$* * * \mathrm{p}<.001 ; * * .001 \leq \mathrm{p}<.01 ; * .01 \leq \mathrm{p}<.05$ 
Appendix 4. 1 Study Cohort Development Chart for Study Population of Elderly Medicare Beneficiaries diagnosed with Prostate Cancer

Men with prostate cancer identified using SEER site recode: 54 and ICD-10-Code: C61.9

$$
\mathrm{N}=358,439
$$

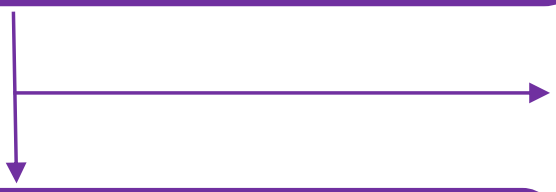

Men diagnosed with prostate cancer as the primary cancer and alive at the time of diagnosis

$$
\mathrm{N}=289,701
$$

Elderly men diagnosed with prostate cancer as the primary cancer at the age 67 years and above, malignant tumor, alive at the time of diagnosis and diagnosed from 2002 to 2009

$$
\mathrm{N}=178,011
$$

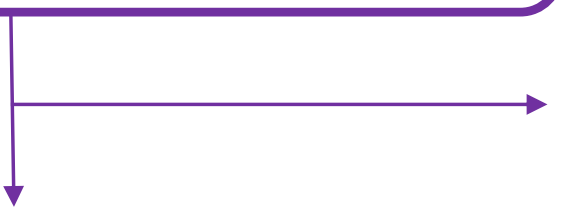

Elderly men diagnosed with prostate cancer as the primary cancer at the age 67 years and above, malignant tumor, alive at the time of diagnosis and diagnosed during 2002 to 2009 with 36-month continuous eligibility in Medicare Part A and Part B and no HMO enrollment during entire 36-month of study period

$$
\mathrm{N}=101,302
$$

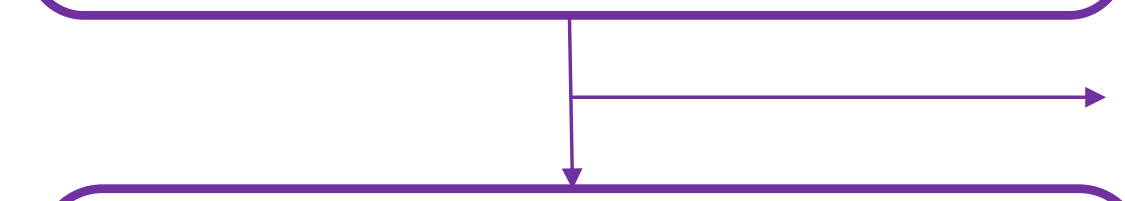

Elderly Men diagnosed with prostate cancer as primary cancer at the age 67 years and above, malignant tumor, alive at the time of diagnosis and diagnosed during 2002 to 2009 with 36-month continuous eligibility in Medicare Part A and Part B and no HMO enrollment during entire 36-month of study period

$$
\mathbf{N}=\mathbf{5 7 , 4 8 9}
$$

Reason for exclusion:

- Men with other primary cancer $(\mathrm{N}=25,785)$

- Men diagnosed with prostate cancer at the time of death or autopsy $(\mathrm{N}=2,944)$

- Men with multiple cancers

Reasons for exclusion:

- Men with carcinoma in-situ ( $N=47)$

- Men diagnosed with prostate cancer at age 66 or below or dead during study periods

Reasons for exclusion:

- No Part A and B enrollment ( $\mathrm{N}=70,559)$

Reasons for exclusion:

- Incident chronic conditions a year preceding and proceeding prostate cancer diagnosis $(\mathrm{N}=43,813)$

- Missing information on race, education, urologist or radiation oncology unit in county $(\mathrm{N}=118)$ 


\begin{tabular}{|c|c|c|c|c|c|c|c|c|c|c|c|c|}
\hline \multicolumn{13}{|c|}{$\begin{array}{l}\text { Appendix 4. } 2 \text { Sensitivity Analysis: Non-cancer hospitalization } \\
\text { Hospitalizations during the Pre- and Post-Cancer Period Elde } \\
\text { Surveillance, Epidemiology and End Results (SEER)-Medicar }\end{array}$} \\
\hline - & \multicolumn{6}{|c|}{$\begin{array}{l}\text { Pre-Cancer Period } \\
\end{array}$} & \multicolumn{6}{|c|}{ Post-Cancer Period } \\
\hline & \multicolumn{2}{|c|}{$\mathbf{t}_{1}$} & \multicolumn{2}{|c|}{$\mathbf{t}_{2}$} & \multicolumn{2}{|c|}{$\mathbf{t}_{3}$} & \multicolumn{2}{|c|}{$\mathbf{t}_{4}$} & \multicolumn{2}{|c|}{$\mathbf{t}_{5}$} & \multicolumn{2}{|c|}{$\mathbf{t}_{6}$} \\
\hline & $\mathbf{N}$ & $\%$ & $\mathbf{N}$ & $\%$ & $\mathbf{N}$ & $\%$ & $\mathbf{N}$ & $\%$ & $\mathbf{N}$ & $\%$ & $\mathbf{N}$ & $\%$ \\
\hline Overall & 2,080 & 3.6 & 1,848 & 3.2 & 2,109 & 3.7 & 2,905 & 5.1 & 2,572 & 4.5 & 2,664 & 4.6 \\
\hline \multicolumn{13}{|c|}{ Types of Chronic Conditions $* * *$} \\
\hline None*** & 332 & 1.3 & 326 & 1.3 & 433 & 1.7 & 660 & 2.6 & 437 & 1.7 & 488 & 1.9 \\
\hline CM only** & 964 & 4.4 & 874 & 4.0 & 942 & 4.3 & 1,311 & 6.0 & 1,203 & 5.5 & 1,220 & 5.6 \\
\hline MH only** & 24 & 3.0 & 21 & 2.6 & 34 & 4.2 & 29 & 3.6 & 30 & 3.7 & 30 & 3.7 \\
\hline RESP only** & 71 & 3.1 & 49 & 2.2 & 58 & 2.5 & 96 & 4.2 & 72 & 3.2 & 68 & 3.0 \\
\hline $\mathrm{CM}+\mathrm{MH}^{* *}$ & 98 & 8.5 & 74 & 6.4 & 86 & 7.5 & 116 & 10.1 & 101 & 8.8 & 102 & 8.9 \\
\hline $\mathrm{CM}+\mathrm{RESP} * *$ & 478 & 9.3 & 415 & 8.1 & 468 & 9.2 & 579 & 11.3 & 601 & 11.8 & 639 & 12.5 \\
\hline All Three**** & 113 & 18.4 & 89 & 14.5 & 88 & 14.3 & 114 & 18.6 & 128 & 20.8 & 117 & 19.1 \\
\hline
\end{tabular}

Notes: Based on 57,489 elderly men, aged 67 years and older, diagnosed with incident prostate cancer between 2002 and 2009 and alive throughout the observation period. Significant differences in NCHs over time were tested with Wald chi-square, after accounting for correlations due to repeated measures. \% represented in the column are column percentage.

Abbreviations: CM: Cardio-metabolic conditions; MH: Mental illness; RESP: Respiratory conditions;

$* * * \mathrm{p}<.001 ; * * .001 \leq \mathrm{p}<.01 ; * .01 \leq \mathrm{p}<.05$ 


\begin{tabular}{|c|c|c|c|}
\hline \multicolumn{4}{|c|}{$\begin{array}{c}\text { Appendix 4. } 3 \text { Sensitivity Analysis: Non-cancer hospitalization } \\
\text { definition 2-Adjusted Odds Ratios and 95\% Confidence Intervals } \\
\text { (CI) of Cancer Diagnosis Period From GEE models on Non-Cancer } \\
\text { Related Hospitalizations Elderly Men with Incident Prostate } \\
\text { Cancer Surveillance, Epidemiology and End Results (SEER)- } \\
\text { Medicare Linked Database-2000-2010 }\end{array}$} \\
\hline \multicolumn{4}{|c|}{ Model 1, adjusting for pre-and post-cancer period } \\
\hline & $\mathbf{O R}$ & $95 \% \mathrm{CI}$ & Sig. \\
\hline \multicolumn{4}{|l|}{ Variable } \\
\hline Pre-cancer & Ref & & \\
\hline Post-cancer & 1.37 & {$[1.32,1.41]$} & $* * *$ \\
\hline \multicolumn{4}{|c|}{$\begin{array}{c}\text { Model 2, adjusting for pre- and post-cancer period + Types of Chronic } \\
\text { Conditions }\end{array}$} \\
\hline & $\overline{\text { AOR }}$ & $95 \%$ CI & Sig. \\
\hline Pre-cancer & Ref & & \\
\hline Post-cancer & 1.38 & {$[1.33,1.42]$} & $* * *$ \\
\hline \multicolumn{4}{|c|}{$\begin{array}{c}\text { Model 3, adjusting for pre- and post-cancer period }+ \text { Types of Chronic } \\
\text { Conditions }+ \text { predisposing, enabling, need, and external environment } \\
\text { characteristics }\end{array}$} \\
\hline & AOR & $95 \% \mathrm{CI}$ & Sig. \\
\hline Pre-cancer & Ref & & \\
\hline Post-cancer & 1.38 & {$[1.33,1.43]$} & $* * *$ \\
\hline
\end{tabular}

Notes: Based on 57,489 elderly men, aged 67 years and older, diagnosed with incident prostate cancer between 2002 and 2009 and alive throughout the observation period. Significant differences are based on the log-likelihood test using a repeated measure generalized estimating equations.

AOR: Adjusted Odds Ratio; GEE: Generalized Estimating Equation; AOR: Adjusted Odds Ratio; Sig: Level of Sig: Significance.

$* * * \mathrm{p}<.001 ; * * .001 \leq \mathrm{p}<.01 ; * .01 \leq \mathrm{p}<.05$ 


\begin{tabular}{|c|c|c|c|c|c|c|}
\hline \multicolumn{7}{|c|}{$\begin{array}{l}\text { Appendix 4. } 4 \text { Sensitivity Analysis: Non-cancer hospitalization definition 2-Predicted Probabilities with 95\% } \\
\text { Confidence Intervals for Types of Chronic Conditions From GEE analysis on Non-Cancer Related } \\
\text { Hospitalizations Elderly Medicare Beneficiaries with Incident Prostate Cancer Surveillance, Epidemiology } \\
\text { and End Results (SEER)-Medicare Linked Database-2000-2010 }\end{array}$} \\
\hline & \multicolumn{2}{|c|}{ Pre-cancer } & \multicolumn{2}{|c|}{ Post-cancer } & \multirow{2}{*}{$\begin{array}{l}\text { Changes in } \\
\text { Probabilities }\end{array}$} & \multirow{2}{*}{$\begin{array}{l}\text { Additive } \\
\text { Effect }\end{array}$} \\
\hline & $\mathbf{P}$ & $95 \% \mathrm{CI}$ & $\mathbf{P}$ & 95\% CI & & \\
\hline \multicolumn{7}{|c|}{ Model 1, adjusted for pre-and post-cancer period } \\
\hline Overall $* * *$ & 0.035 & {$[0.034,0.036]$} & 0.047 & {$[0.046,0.048]$} & \multicolumn{2}{|l|}{0.012} \\
\hline \multicolumn{7}{|c|}{ Model 2, adjusted for pre- and post-cancer period + Types of Chronic Conditions } \\
\hline Overall $* * *$ & 0.028 & {$[0.027,0.029]$} & 0.038 & {$[0.037,0.039]$} & \multicolumn{2}{|l|}{0.010} \\
\hline \multicolumn{7}{|c|}{ Types of Chronic Conditions ${ }^{* * * *}$} \\
\hline None & 0.018 & {$[0.017,0.018]$} & 0.031 & {$[0.030,0.032]$} & 0.013 & Ref \\
\hline CM only & 0.044 & {$[0.043,0.046]$} & 0.077 & {$[0.075,0.079]$} & 0.033 & 0.020 \\
\hline MH only & 0.032 & {$[0.027,0.037]$} & 0.055 & {$[0.047,0.063]$} & 0.023 & 0.010 \\
\hline RESP only & 0.029 & {$[0.026,0.031]$} & 0.05 & {$[0.045,0.054]$} & 0.021 & 0.008 \\
\hline $\mathrm{CM}+\mathrm{MH}$ & 0.075 & {$[0.069,0.081]$} & 0.127 & {$[0.117,0.137]$} & 0.052 & 0.039 \\
\hline $\mathrm{CM}+\mathrm{RESP}$ & 0.087 & {$[0.084,0.091]$} & 0.146 & {$[0.141,0.151]$} & 0.059 & 0.046 \\
\hline All Three & 0.150 & {$[0.138,0.162]$} & 0.239 & {$[0.222,0.256]$} & 0.089 & 0.076 \\
\hline \multicolumn{7}{|c|}{$\begin{array}{c}\text { Model } 3 \text { adjusted for pre- and post-cancer period }+ \text { Types of Chronic Conditions }+ \text { predisposing, enabling, need, } \\
\text { and external environment characteristics }\end{array}$} \\
\hline Overall**** & 0.027 & {$[0.026,0.028]$} & 0.036 & {$[0.036,0.038]$} & 0.009 & \\
\hline \multicolumn{7}{|c|}{ Types of Chronic Conditions ${ }^{* * * *}$} \\
\hline None & 0.016 & {$[0.015,0.016]$} & 0.021 & {$[0.020,0.022]$} & 0.008 & Ref \\
\hline CM only & 0.039 & {$[0.038,0.041]$} & 0.053 & {$[0.052,0.055]$} & 0.014 & 0.008 \\
\hline MH only & 0.026 & {$[0.022,0.030]$} & 0.036 & {$[0.030,0.041]$} & 0.010 & 0.004 \\
\hline RESP only & 0.021 & {$[0.019,0.023]$} & 0.029 & {$[0.026,0.032]$} & 0.008 & 0.002 \\
\hline $\mathrm{CM}+\mathrm{MH}$ & 0.056 & {$[0.051,0.061]$} & 0.075 & {$[0.069,0.082]$} & 0.020 & 0.014 \\
\hline $\mathrm{CM}+\mathrm{RESP}$ & 0.066 & {$[0.062,0.069]$} & 0.088 & {$[0.084,0.092]$} & 0.023 & 0.017 \\
\hline All Three & 0.107 & {$[0.097,0.116]$} & 0.141 & {$[0.129,0.153]$} & 0.035 & 0.029 \\
\hline
\end{tabular}

Notes: Based on 57,489 elderly men, aged 67 years and older, diagnosed with incident prostate cancer between 2002 and 2009 and alive throughout the observation period. Significant differences are based on the log-likelihood test using a repeated measure generalized estimating equations.

Abbreviations: CM: Cardio-metabolic conditions; MH: Mental health conditions; RESP: Respiratory conditions; Sig: Level of Significance;

$* * * \mathrm{p}<.001 ; * * .001 \leq \mathrm{p}<.01 ; * .01 \leq \mathrm{p}<.05$ 


\section{Chapter 5}

Discussion of Findings and Research Implications

\subsection{SUMMARY OF FINDINGS \& DISCUSSION}

With an increase in the cancer-related survival and risk of non-cancer mortality among elderly men with prostate cancer, the Office of Cancer Survivorship (OCS) and Institute of Medicine (IOM) have prioritized the co-management of chronic conditions and cancer. The current study, which focuses on the role of chronic conditions in cancer treatment, cancer and non-cancer outcomes, is timely, unique and in alignment with the scope of the OCS and IOM. In the current study, one in two elderly ( $\geq 67$ years) feefor-service Medicare beneficiaries with incident prostate cancer had a pre-existing chronic condition, consistent with the published studies. Among those with pre-existing chronic conditions, the cardiometabolic conditions were highly prevalent (53.8\%). These findings suggest that elderly men with prostate cancer have a significant illness burden.

In the current study, types of pre-existing chronic conditions were not associated with cancer stage at diagnosis. One in 10 elderly Medicare beneficiaries had advanced prostate cancer at diagnosis. Elderly men with none of three types of chronic conditions were more likely to be diagnosed with advanced prostate cancer as compared to those with all the three types of chronic conditions. No statistically significant associations between other types of chronic conditions and the risk of advanced stage prostate cancer were observed. These findings suggest that the strategies for reducing the risk of advanced prostate cancer at diagnosis should focus on elderly men with none of three common types of chronic conditions.

\section{The initial prostate cancer treatment rates were high even among elderly men with incident} localized prostate cancer. In the study cohort, three in four elderly Medicare beneficiaries diagnosed with incident localized prostate cancer received either RP, RT or hormone therapy during the first six-month after cancer diagnosis. Although there is no evidence that the initial cancer treatment can be beneficial the in long-term for elderly men with prostate cancer and pre-existing chronic conditions, many received treatment, suggesting an overuse of $R P$ and $R T$ among elderly men with localized incident prostate cancer.

The types of chronic conditions were associated with some types of the initial cancer treatment. As compared to elderly men with all the three types of chronic conditions, those with none of three types of 
chronic conditions were more likely to receive RP/RT, men in all other chronic condition categories (except "mental health and respiratory conditions" and "only mental health conditions") were more likely to receive RT. There were no differences in the receipt of hormone therapy by the types of chronic conditions. Again, prior research has shown that elderly men with cardio-metabolic conditions may not benefit from cancer treatment as these men are at high risk for non-cancer related mortality $(8,50)$. These findings reinforce the need for specific clinical guidelines for the treatment of cancer in presence of particular types of chronic conditions.

\section{The types of chronic conditions were associated with bowel, sexual and urinary dysfunction. As} compared to those with all the three types of conditions, elderly men with none of the three types of chronic conditions or only respiratory conditions or only cardio-metabolic were less likely to have bowel and urinary dysfunctions. Whereas, as compared to elderly men with all the three types of conditions, those with none of the three types of chronic conditions, and only respiratory conditions were more likely to have sexual dysfunctions. The reasons for why these particular combinations are associated with lower risk of bowel and urinary dysfunctions were not known. Future research needs to explore the possible pathophysiological link between types of chronic conditions and bowel and urinary dysfunction.

The treatment of pre-existing T2DM with metformin during the year before the incident prostate cancer diagnosis reduced the risk for advanced prostate cancer at diagnosis. Among elderly men with prostate cancer and T2DM, metformin use during the year before diagnosis of incident prostate cancer was associated with a statistically significant reduction in the risk of advanced prostate cancer at diagnosis, after controlling for the observed selection bias between metformin users and non-users and other independent variables. This finding is consistent with the pre-clinical evidence. If the findings are replicated by other observational studies and RCTs, metformin can be used as a preventive therapy for reducing the risk of advanced prostate cancer.

Metformin users were more likely to receive RP as the initial cancer treatment as compared to non-users. The reasons for a higher rate of RP among metformin users are not clear. Future research needs to explore the reasons as to why metformin users were more likely to receive aggressive treatments. 
Prostate cancer diagnosis was associated with an increased risk of non-cancer hospitalizations among elderly men with incident prostate cancer. Elderly Medicare beneficiaries with incident prostate cancer had higher rates of non-cancer hospitalizations during the post-cancer period as compared to the pre-cancer period, even after controlling for a comprehensive list of risk factors. The highest rates of noncancer hospitalizations were observed during first four months after the diagnosis of prostate cancer. Collectively, these findings suggest the need for further research on the relationship between the appropriate management of pre-existing chronic conditions during the pre-cancer period and the risk of non-cancer hospitalizations during the post-cancer period. Along with cancer care, better surveillance efforts and closer monitoring of chronic conditions may be needed, especially at the time of incident cancer diagnosis among elderly men, to reduce the risk of excess non-cancer hospitalizations.

The magnitude of the relationship between the diagnosis of prostate cancer and non-cancer hospitalizations varied by the types of pre-existing chronic conditions. Those with all the three types of conditions, both cardio-metabolic conditions and respiratory conditions, and both cardio-metabolic and mental-health conditions had a greater increase in the risk of non-cancer-hospitalizations (between the precancer and post-cancer period) as compared to those with none of the three types of chronic conditions. The study also has a key methodological implication for estimating cancer-related healthcare utilization. Typically, cancer-related annual healthcare utilizations among men with prostate cancer are estimated by subtracting the total healthcare utilization in a year before cancer diagnosis from the total utilization after in a year after cancer diagnosis (5-9). Such method of estimating cancer-related healthcare utilizations may overestimate prostate-cancer-related utilizations as elderly men with incident prostate cancer had higher rates of non-cancer hospitalizations during post-cancer as compared to pre-cancer period.

\subsection{IMPLICATIONS FOR FUTURE RESEARCH}

Historically, the role of chronic conditions in cancer has been examined using comorbidity indices or the number of chronic conditions. The current study is the first of its kind to provide comprehensive information on the role of specific types of chronic conditions and their combinations in prostate cancer diagnosis, treatment, and cancer and non-cancer outcomes. The study findings provide some actionable evidence that can be utilized in real-world treatment decisions. One of the major research implications is to 
conduct future studies among individuals with other types of cancers with better survival profile such as the localized breast, colon, rectum, melanoma, testis, thyroid, urinary bladder and uterine corpus cancers.

In the current study it was observed that elderly men without the commonly prevalent chronic conditions had advanced prostate cancer at diagnosis. Future research studies should examine the impact of less common conditions such as inflammatory related conditions, smoking associated disorders, sexually transmitted disease on prostate cancer risk, as these conditions also share common risk factors for developing prostate cancer.

The current study found a reduced risk of advanced prostate cancer diagnosis with metformin use among elderly men with T2DM and prostate cancer. Well-designed pragmatic trials are needed to provide evidence on the effectiveness of metformin use.

The SEER authority advised against the use of the prostate specific antigen (PSA) as the PSA levels were not accurately recorded over the years. In the current study, PSA levels could not be incorporated to examine cancer stage at diagnosis. Therefore, future research should focus on the reliability and validity of using the PSA levels from the real-world electronic medical records.

As the elderly men with prostate cancer at higher risk for non-cancer hospitalizations during the post-cancer period as compared to the pre-cancer period, future studies should examine the reasons behind the increased risk.

\subsection{STUDY LIMITATIONS}

The current study has some limitations. First, the study cohort was restricted to elderly men enrolled in fee-for-service Medicare services only residing in the SEER-regions, therefore, study findings cannot be generalized to younger men, enrolled in managed care plans or commercial plans, and those residing in non-SEER regions. In addition, in the second aim, our study restricted to elderly men with a history of diabetes, the findings cannot be generalized to those with incidence of prostate cancer and no prior history of diabetes. The clinical severity of the chronic conditions could not be measured.

Furthermore, the current study utilized the registry-linked administrative claims data which have either no information or limited validity of codes to identify body-mass index, exercise, and smoking status, which 
may be associated severity, advanced prostate cancer at diagnosis, the initial treatment receipt and clinical outcome among elderly men with prostate cancer. In addition, the types of chronic conditions were considered as incurable conditions. The cardio-metabolic conditions and respiratory conditions can be considered as incurable conditions, however, the mental-health conditions such as depression can be cured with proper medications and other clinical approaches. Therefore, the chronic condition classification might overestimate the presence of such conditions. Given this limitation, the presence of each of those conditions was identified in the immediate periods before diagnosis of cancer.

Despite these limitations, the current study contributed to the nascent literature on the role of selected chronic conditions and their combinations on the stage of cancer, the initial cancer treatment, cancer- and non-cancer outcomes among elderly men with incident prostate cancer using the largest population-based cancer registries-Medicare-linked database of the US. 


\subsection{REFERENCES}

1. Chamie K, Daskivich TJ, Kwan L, Labo J, Dash A, Greenfield S, et al. Comorbidities, treatment and ensuing survival in men with prostate cancer. J Gen Intern Med. 2012 May;27(5):492-9.

2. Nguyen PL, Chen MH, Hoffman KE, Chen RC, Hu JC, Bennett CL, et al. Cardiovascular comorbidity and treatment regret in men with recurrent prostate cancer. BJU Int. 2012 Jul;110(2):201-5.

3. Vavassori V, Fiorino C, Rancati T, Magli A, Fellin G, Baccolini M, et al. Predictors for rectal and intestinal acute toxicities during prostate cancer high-dose 3D-CRT: results of a prospective multicenter study. Int J Radiat Oncol Biol Phys. 2007 Apr 1;67(5):1401-10.

4. Tucker SL, Dong L, Bosch WR, Michalski J, Winter K, Mohan R, et al. Late rectal toxicity on RTOG 94-06: analysis using a mixture Lyman model. Int J Radiat Oncol Biol Phys. 2010 Nov 15;78(4):1253-60.

5. Sanyal C, Aprikian AG, Chevalier S, Cury FL, Dragomir A. Direct cost for initial management of prostate cancer: a systematic review. Curr Oncol. 2013 Dec;20(6):e522-31.

6. Burkhardt JH, Litwin MS, Rose CM, Correa RJ, Sunshine JH, Hogan C, et al. Comparing the Costs of Radiation Therapy and Radical Prostatectomy for the Initial Treatment of Early-Stage Prostate Cancer. Journal of Clinical Oncology. 2002 June 15;20(12):2869-75.

7. Zeliadt SB, Etzioni R, Ramsey SD, Penson DF, Potosky AL. Trends in treatment costs for localized prostate cancer: the healthy screenee effect. Med Care. 2007 Feb;45(2):154-9.

8. Roehrborn CG, Albertsen P, Stokes ME, Black L, Benedict A. First-year costs of treating prostate cancer: estimates from SEER-Medicare data. Prostate Cancer Prostatic Dis. 2009;12(4):355-60.

9. Penberthy L, Retchin SM, McDonald MK, McClish DK, Desch CE, Riley GF, et al. Predictors of Medicare costs in elderly beneficiaries with breast, colorectal, lung, or prostate cancer. Health Care Manag Sci. 1999 Jul;2(3):149-60. 